\title{
Kodama time, entropy bounds, the Raychaudhuri equation, and the quantum interest conjecture
}

by

\author{
Gabriel Abreu
}

A thesis

submitted to the Victoria University of Wellington in fulfilment of the requirements for the degree of

Doctor of Philosophy in Mathematics.

Victoria University of Wellington 2011 



\begin{abstract}
eneral Relativity, while ultimately based on the Einstein equations, $\checkmark$ also allows one to quantitatively study some aspects of the theory without explicitly solving the Einstein equations. These geometrical notions of the theory provide an insight to the nature of more general spacetimes. In this thesis, the Raychaudhuri equation, the choice of the coordinate system, the notions of surface gravity and of entropy, and restrictions on negative energy densities on the form of the Quantum Interest Conjecture, will be discussed.

First, using the Kodama vector, a geometrically preferred coordinate system is built. With this coordinate system the usual quantities, such as the Riemann and Einstein tensors, are calculated.

Then, the notion of surface gravity is generalized in two different ways. The first generalization is developed considering radial ingoing and outgoing null geodesics, in situations of spherical symmetry. The other generalized surface gravity is a three-vector obtained from the spatial components of the redshifted four acceleration of a suitable set of fiducial observers. This vectorial surface gravity is then used to place a bound on the entropy of both static and rotating horizonless objects. This bound is obtain mostly by classical calculations, with a minimum use of quantum field theory in curved spacetime.

Additionally, several improved versions of the Raychaudhuri equation are developed and used in different scenarios, including a two congruence generalization of the equation.

Ultimately semiclassical quantum general relativity is studied in the specific form of the Quantum Inequalities, and the Quantum Interest Con-
\end{abstract}


jecture. A variational proof of a version of the Quantum Interest Conjecture in $(3+1)$-dimensional Minkowski space is provided. 


\section{Acknowledgments}

$\mathrm{T}$

There are not enough pages to thank every person who has influenced me during the development of this doctoral thesis.

Certainly this work, from its inception to its final shape, would not be possible without the hard work and patience of Professor Matt Visser. His invaluable advice and support throughout these past three years will always be appreciated.

I cannot thank enough my family, in all of its extension, for their wholesome support. Specially I would not be here without the help and love of my mother, Lilia Castellanos, who has taught me so much. I also want to thank Marlenis Castellanos, Gloria Lopez and Rafael Castellanos for their lessons and help through life.

A lot of the work and time spent on this research would not have been as enjoyable without the companionship and help of Kym Thomas. Additionally, I wish to thank Jozef Skakala for being an excellent office and travel mate for the last years. I am grateful to Bethan Cropp and Nicole Walters for quickening the completion of this work. Also, I would like to thank Hee Che, Sophia Yong, Freeman Tong, Ilias Pechlivanidis and Céline Cattoën-Gilbert for their friendship.

I am also very grateful to the Victoria PhD Scholarship and Marsden fund that have supported me financially during my work at the Victoria University of Wellington and related travel and conferences.

Finally I dedicate this work to Jose Gabriel Abreu R. and Aura Abreu R. who will always be with me. 



\section{Contents}

$\begin{array}{lll}1 & \text { Introduction } & 1\end{array}$

2 Kodama time 5

2.1 Warped product spacetimes . . . . . . . . . . . . . 7

2.2 The Kodama miracle . . . . . . . . . . . . . . . . . . . . . 10

2.3 Generalized Kodama flux . . . . . . . . . . . . . . . . . 13

2.4 Kodama time . . . . . . . . . . . . . . . . . . . 14

2.5 Riemann tensor . . . . . . . . . . . . . . . . . . . . . 18

2.6 Einstein tensor . . . . . . . . . . . . . . . . . . . . . . . 19

2.7 Co-ordinate based version of Kodama's conservation law . . 21

2.8 Brown-York quasi-local mass . . . . . . . . . . . . . . 22

2.9 Surface gravity . . . . . . . . . . . . . . . . 24

2.9 .1 Surface gravity from fiducial observers . . . . . . . . 24

2.9 .2 Surface gravity from radial null geodesics. . . . . . . 26

2.10 The evolving horizon . . . . . . . . . . . . . . . 28

2.11 Discussion . . . . . . . . . . . . . . . . . . . . . . . . . . 29

3 Entropy bounds 33

$3.1 \quad$ Entropy bounds for static uncollapsed matter . . . . . . . . . 34

3.1.1 Tolman mass and generalized surface gravity . . . . 34

3.1 .2 Entropy bounds and the Unruh effect . . . . . . . . . 36

3.1 .3 Spherical symmetry . . . . . . . . . . . . . . 39

$3.1 .4 \quad$ Black hole region $\ldots \ldots \ldots \ldots$. . . . . . . . . . 41

3.2 Entropy bounds for uncollapsed rotating

bodies....................... 43

3.2 .1 Metric . . . . . . . . . . . . . . . 45

3.2 .2 Matter . . . . . . . . . . . . . . . 46

3.2 .3 Thermodynamic equilibrium . . . . . . . . . . . 47

3.2 .4 Entropy current . . . . . . . . . . . . . . . . . 48

3.2 .5 Classical entropy bound . . . . . . . . . . . . 49 
3.2 .6 Unruh temperature . . . . . . . . . . . . . . . 52

3.2 .7 The semiclassical bound . . . . . . . . . . . . . . . . . 54

3.3 Discussion . . . . . . . . . . . . . . . . . . 55

4 The Raychaudhuri equation $\quad 59$

$4.1 \quad$ Single unit timelike vector field . . . . . . . . . . . . . . . . . . 60

$4.2 \quad$ Applications . . . . . . . . . . . . . . . . . . . 61

$4.2 .1 \quad$ Vorticity-free congruence . . . . . . . . . . . . . 62

4.2 .2 ADM formalism . . . . . . . . . . . . . . . 63

4.2 .3 Static spacetimes . . . . . . . . . . . . . . . . . . 64

$4.2 .4 \quad$ Stationary spacetime - Killing congruence . . . . . . 65

4.2 .5 Stationary axisymmetric spacetimes . . . . . . . . . . 67

4.3 Single unit spacelike vector field . . . . . . . . . . . . . 67

4.4 Single non-normalized vector field . . . . . . . . . . . . . . 69

4.5 Two non-normalized vector fields. . . . . . . . . . . . . . . 71

4.5 .1 Motivation . . . . . . . . . . . . . . . . . . . 71

4.5 .2 Construction . . . . . . . . . . . . . . . . 73

4.5 .3 Generalizing the Landau-Lifshitz result . . . . . . . 74

4.5 .4 Landau-Lifshitz in axial symmetry. . . . . . . . . . 75

4.6 Discussion . . . . . . . . . . . . . . . . . . . . 77

5 The quantum interest conjecture in (3+1) dimensional Minkowski

space 79

5.1 Quantum Inequalities and the Quantum interest Conjecture 82

5.1 .1 The Quantum Inequalities . . . . . . . . . . . . . . . 82

$5.1 .2 \quad$ The QIC as an eigenvalue problem . . . . . . . . . . 84

$5.2 \quad 1+1$ dimensional Minkowski space $\ldots \ldots \ldots \ldots \ldots$

5.2 .1 Gaussian wave-function . . . . . . . . . . . . . . 86

5.2 .2 Generic class of test functions . . . . . . . . . . . . 87

$5.3 \quad 3+1$ dimensional Minkowski space $\ldots \ldots \ldots \ldots$. . . . . . . 92

5.3 .1 Gaussian wave-function . . . . . . . . . . . . . . . . . 92

5.3 .2 Generic class of test functions . . . . . . . . . . . . . . 93 
5.4 Higher dimensions $\ldots \ldots \ldots$. . . . . . . . . . . . 95

5.5 Discussion . . . . . . . . . . . . . . . . . . . . 98

\begin{tabular}{lll}
\hline 6 & Conclusions & 101
\end{tabular}

\begin{tabular}{ll}
\hline Appendices & 103
\end{tabular}

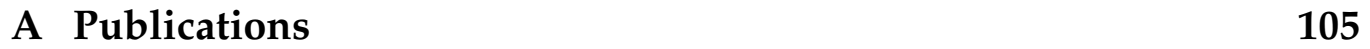

A.1 Quantum interest in (3+1) dimensional

Minkowski space . . . . . . . . . . . . . . . . . . 105

A.2 Kodama time: Geometrically preferred foliations of spherically symmetric spacetimes . . . . . . . . 106

A.3 Tolman mass, generalized surface gravity, and entropy bounds 107

A.4 Entropy bounds for uncollapsed rotating bodies . . . . . . . . . . . . . . . . . . . 107

A.5 Some generalizations of the Raychaudhuri equation . . . . . 108

$\begin{array}{ll}\text { Bibliography } & 109\end{array}$

Index 122 



\title{
7 \\ Introduction
}

\begin{abstract}
Cravitation stands alone as the only fundamental force not yet deJcribed by a full quantum theory. Nevertheless the classical and semi-classical theories of General Relativity provide a good description of several gravitational phenomena. Furthermore, it is even possible to extract information from the theory without explicitly solving the Einstein equations. Focusing on the geometrical aspects of the theory allows us to study more general scenarios regardless of the existence or not of explicit solutions for the Einstein equations for the specific case.

Among these geometrical aspects, the different choices of the coordinate system can help to understand different characteristics of the same problem. Furthermore, once there is a physical or geometrical reason for the choice of the coordinates, it is possible to study the system from a conveniently specialized vantage point. For instance, the surface gravity, although traditionally obtained from the full solution of the Einstein equations, can be calculated without using such solutions, thereby generalizing it. These generalizations of the surface gravity prove to be important in alternative derivations of the Hawking effect, as well as in the construction of minimalist entropy bounds of static, and rotating uncollapsed bodies.

Another important, and very useful geometrical tool in General Relativity is the Raychaudhuri equation. Indispensable in the interpretation of the Energy Conditions, the equation contains more information than is tra-
\end{abstract}


ditionally attributed to it. The extra information can be extracted making simple changes in the way the equation is usually arranged.

Semi-classical General Relativity can also be studied without explicitly solving the Einstein equations, however the existence of quantized matter predicts violations of the classical energy conditions. These violations of the energy conditions are related to unbounded amounts of negative energy, and potentially lead to exotic phenomena such as time machines and warp drives. Nevertheless, these exotic phenomena can be prevented by the theory itself through the so called Quantum Inequalities and Quantum Interest Conjecture.

The layout of this thesis is as follows: Chapter 2 shows how a geometrically preferred coordinate system can be constructed using the Kodama vector in a spherically symmetric spacetime. We first extract as much information as possible by invoking the "warped product" structure of spherically symmetric spacetime to study the Kodama vector, and the associated Kodama energy flux, in a coordinate independent manner. Using this formalism we construct a general class of conservation laws, generalizing Kodama's energy flux.

We then demonstrate that a preferred time coordinate - which we shall call Kodama time - can be introduced by taking the additional step of applying the Clebsch decomposition theorem to the Kodama vector. We thus construct a geometrically preferred coordinate system for any timedependent spherically symmetric spacetime, and explore its properties. We also study the geometrically preferred fiducial observers, and demonstrate that it is possible to define and calculate a generalized notion of surface gravity that is valid throughout the entire evolving spacetime. Furthermore, by building and suitably normalizing set of radial null geodesics, we can show that this generalized surface gravity passes several consistency tests and has a physically appropriate static limit.

In Chapter 3 we develop a series of entropy bounds that are very similar in spirit to the holographic bound, and closely related to entanglement 
entropy, for both static and rotating distributions of uncollapsed matter. For the static geometry, the quasilocal Tolman mass contained within a volume can be reduced to a Gauss-like surface integral involving the flux of a suitably defined generalized surface gravity. By introducing some basic thermodynamics, and invoking the Unruh effect, one can then develop elementary bounds on the quasilocal entropy.

For the rotating body things are more complicated, and the Tolman mass is not the quantity that has a useful bound. However, after assuming stationarity, azimuthal symmetry and rigid rotation, we are still able to bound the quasilocal entropy using only classical notions. Ultimately, in a similar fashion to the static case, the entropy is also bounded by the total area of the rotating uncollapsed body.

Chapter 4 deals with the Raychaudhuri equation, and some of its generalizations in different geometries. First an improved version of the standard timelike Raychaudhuri equation is developed, where several key terms are lumped together as a divergence. This already has a number of interesting applications, both within the ADM formalism and elsewhere. Second, a spacelike version of the Raychaudhuri equation is briefly discussed. Third, a version of the Raychaudhuri equation is developed that does not depend on the use of normalized congruences. This leads to useful formulae for the "diagonal" part of the Ricci tensor. Fourth, a "two vector" version of the Raychaudhuri equation is developed that uses two congruences to effectively extract "off diagonal" information concerning the Ricci tensor.

Finally, in Chapter 5 we go deeper into the semi-classical part of the theory of General Relativity, where the matter is described by quantum field theory in curved spacetimes. It is well known that quantum matter violates the classical energy conditions, allowing arbitrary large amounts of negative energy densities at individual points. Nevertheless, the theory itself provides us with restrictions on the distribution of exotic matter, in the form of the Quantum Inequalities and the Quantum Interest 
Conjecture. Both assertions can be reduced to statements concerning the existence or non-existence of bound states for a certain one-dimensional quantum mechanical pseudo-Hamiltonian. Using this approach, we shall provide a simple variational proof of one version of the Quantum Interest Conjecture in $(3+1)$-dimensional Minkowski space. 


\section{2 \\ Kodama time}

B lack holes are an iconic part of Einstein's general relativity. While we have a very detailed understanding of static and stationary black holes (the Schwarzschild, Reissner-Nordström, Kerr, and Kerr-Newman black holes), the situation with regard to evolving black holes, (evolving either due to accretion or Hawking radiation or both), is much more opaque. In particular, the rather limited number of currently known exact evolving solutions (Oppenheimer-Snyder collapse, the Vaidya solution) makes it much more difficult to fully describe an evolving black hole in any analytic detail. A fundamental feature of the geometry of an evolving time-dependent spacetime is the lack of any (asymptotically timelike) Killing vector field, which seems to leave us without a preferred time coordinate with which to study the problem.

In 1980 Kodama made significant progress in this regard when he constructed a geometrically natural divergence-free preferred vector field that is guaranteed to exist in any time-dependent spherically symmetric spacetime [1]. This so-called "Kodama vector" defines a natural timelike direction in the region exterior to the black hole, and additionally induces an unexpected conserved current, but does not (in and of itself) define any naturally preferred time coordinate. By considering the "warped product" form of the spacetime metric for any spherically symmetric geometry we are able to investigate the Kodama vector (and the associated Kodama 
energy flux) in a coordinate independent manner. In particular we can use this formalism to easily generate a generalized Kodama flux.

By furthermore taking the extra step of invoking the Clebsch decomposition [2, 3, 4] on the (1+1) dimensional radial-temporal plane, we shall demonstrate that the Kodama vector field can indeed be used to construct a preferred time coordinate, and more importantly a preferred coordinate system.

The absence of any (asymptotically timelike) Killing vector in evolving spacetimes has made it difficult to achieve any consensus about best way to define such fundamental quantities as the surface gravity. Over the years, several different attempts have been made to extend the concept of surface gravity from static (and stationary) to time-dependent spacetimes. For instance, Hayward [5, 6, 7, 8] uses the Kodama vector itself as a substitute for the Killing vector, since it certainly provides a preferred direction and it is parallel to the Killing vector in the static case (as well as at spatial infinity if one assumes the evolving spacetime is asymptotically flat). Others (see [9, 10, 11, 12, 13, 14, 15] and references therein) have appealed to the freedom of normalization of the null geodesics to ensure their definitions reduce to known results in the static case [16].

The layout of this chapter is as follows: In section 2.1 we briefly summarize key properties of "warped product" spacetimes. In section 2.2 we present a quick review of the Kodama vector and Kodama's unexpected conservation law, and then significantly generalize Kodama's energy flux in section 2.3. Next, in section 2.4, a Clebsch decomposition of the Kodama vector is made - in order to build a natural geometrically preferred coordinate system for any spherically symmetric time-dependent spacetime. In sections 2.5 and 2.6 we explore the Riemann and Einstein tensors in this geometrically preferred coordinate system, being careful to connect the discussion back to the general "warped product" formalism of section 2.1. Furthermore, in section 2.7 we review Kodama's conservation law in these preferred coordinates. In section 2.8 we calculate the Brown-York 
quasi-local mass, and in section and 2.9 present our extended definition of surface gravity . Section 2.10 deals with the naturally induced notions of apparent and trapping horizon. Lastly, we add a brief discussion.

\subsection{Warped product spacetimes}

Any (possibly time-dependent) spherically symmetric metric can be written in the form

$$
\begin{aligned}
\mathrm{d} s^{2} & =g_{a b} \mathrm{~d} x^{a} \mathrm{~d} x^{b} \\
& ={ }^{B} g_{i j}(x) \mathrm{d} x^{i} \mathrm{~d} x^{j}+r(x)^{2}\left\{\mathrm{~d} \theta^{2}+\sin ^{2} \theta \mathrm{d} \phi^{2}\right\} \\
& ={ }^{B} g_{i j}(x) \mathrm{d} x^{i} \mathrm{~d} x^{j}+r(x)^{2}{ }^{F} g_{\alpha \beta} \mathrm{d} x^{\alpha} \mathrm{d} x^{\beta} .
\end{aligned}
$$

Here the two coordinates $x^{i}$ run over the radial-temporal plane, while the two coordinates $x^{\alpha}(\theta$ and $\phi)$ run over the surfaces of spherical symmetry. Also, ${ }^{B} g_{i j}$ and ${ }^{F} g_{\alpha \beta}$ are the "base space" and "fibre" metrics, respectively. The discussion can be generalized to $(d+1)$ dimensions with $d-1$ dimensional spherical symmetry, but for now we are just working in $(3+1)$ dimensions. (For higher-dimensional generalizations in a Gauss-Bonnet context see [17, 18].) Independent of the total dimensionality, ${ }^{B} g_{i j}$ is a $(1+1)$ dimensional Lorentzian metric.

Geometrically this is called a "warped product" manifold, with the radial-temporal plane being referred to as the "base space", the surfaces of spherical symmetry being referred to as the "fibres", and the function $r(x)$ which depends only on the base space coordinates being referred to as the "warp factor". It is a standard computation to show that (up to the usual permutation symmetries for the indices) the only non-zero components of the Riemann tensor (in any warped product spacetime) are

$$
\begin{aligned}
R_{i j k l} & ={ }^{B} R_{i j k l} ; \\
R_{i \alpha j \beta} & =-r\left\{\nabla_{i} \nabla_{j} r\right\}{ }^{F} g_{\alpha \beta} ; \\
R_{\alpha \beta \mu \nu} & =r^{2}\left\{{ }^{F} R_{\alpha \beta \mu \nu}-|\nabla r|^{2}\left({ }^{F} g_{\alpha \mu}{ }^{F} g_{\beta \nu}-{ }^{F} g_{\alpha \nu}{ }^{F} g_{\beta \mu}\right)\right\} .
\end{aligned}
$$


An abstract computation along these lines can be found in $\mathrm{O}^{\prime} \mathrm{Neill}$ [19, page 210] while more explicit computations can be found in [20, 21, 22]. Note that the covariant derivatives appearing above are covariant derivatives in the base space. But because $r(x)$ depends only on the $(1+1)$ dimensional base space coordinates, and because of the specific form of the warped product metric, these derivatives can be "bootstrapped" to covariant derivatives in the total warped product spacetime.

In the specific situation we are interested in the base space is two dimensional, so in terms of the Ricci scalar of the radial-temporal plane we have the specific simplification

$$
{ }^{B} R_{i j k l}=\frac{{ }^{B} R}{2}\left({ }^{B} g_{i k}{ }^{B} g_{j l}-{ }^{B} g_{i l}{ }^{B} g_{j k}\right) .
$$

Furthermore the fibre is a constant curvature sphere of radius unity, so

$$
{ }^{F} R_{\alpha \beta \mu \nu}=\left({ }^{F} g_{\alpha \mu}{ }^{F} g_{\beta \nu}-{ }^{F} g_{\alpha \nu}{ }^{F} g_{\beta \mu}\right)
$$

Thus we now have on purely geometrical grounds

$$
\begin{aligned}
R_{i j k l} & =\frac{{ }^{B} R}{2}\left({ }^{B} g_{i k}{ }^{B} g_{j l}-{ }^{B} g_{i l}{ }^{B} g_{j k}\right) ; \\
R_{i \alpha j \beta} & =-r\left\{\nabla_{i} \nabla_{j} r\right\}{ }^{F} g_{\alpha \beta} ; \\
R_{\alpha \beta \mu \nu} & =r^{2}\left\{1-|\nabla r|^{2}\right\}\left({ }^{F} g_{\alpha \mu}{ }^{F} g_{\beta \nu}-{ }^{F} g_{\alpha \nu}{ }^{F} g_{\beta \mu}\right) .
\end{aligned}
$$

It is also common in spherical symmetry to define the Hawking-Israel/ Hernandez-Misner/ Misner-Sharp quasi-local mass [23, 24] by

$$
1-\frac{2 m}{r}=|\nabla r|^{2}
$$

where both $m\left(x^{i}\right)$ and $r\left(x^{i}\right)$ are scalar functions on the base space. We now have

$$
\begin{aligned}
R_{i j k l} & =\frac{{ }^{B} R}{2}\left({ }^{B} g_{i k}{ }^{B} g_{j l}-{ }^{B} g_{i l}{ }^{B} g_{j k}\right) \\
R_{i \alpha j \beta} & =-r\left\{\nabla_{i} \nabla_{j} r\right\}{ }^{F} g_{\alpha \beta} ; \\
R_{\alpha \beta \mu \nu} & =2 m r \quad\left({ }^{F} g_{\alpha \mu}{ }^{F} g_{\beta \nu}-{ }^{F} g_{\alpha \nu}{ }^{F} g_{\beta \mu}\right) .
\end{aligned}
$$


It is often useful to go to an orthonormal basis, in which case

$$
\begin{aligned}
R_{\hat{i} \hat{j} \hat{j} \hat{l}} & =\frac{{ }^{B} R}{2}\left(\delta_{\hat{i} \hat{k}} \delta_{\hat{j} \hat{l}}-\delta_{\hat{i} \hat{l}} \delta_{\hat{j} \hat{k}}\right) \\
R_{\hat{i} \hat{\alpha} \hat{j} \hat{\beta}} & =-\frac{\left\{\nabla_{\hat{i}} \nabla_{\hat{j}} r\right\}}{r} \delta_{\hat{\alpha} \hat{\beta}} ; \\
R_{\hat{\alpha} \hat{\beta} \hat{\mu} \hat{\nu}} & =\frac{2 m}{r^{3}}\left(\delta_{\hat{\alpha} \hat{\mu}} \delta_{\hat{\beta} \hat{\nu}}-\delta_{\hat{\alpha} \hat{\nu}} \delta_{\hat{\beta} \hat{\mu}}\right) .
\end{aligned}
$$

For the Ricci tensor we have

$$
\begin{aligned}
R_{i j} & =\frac{{ }^{B} R}{2}{ }^{B} g_{i j}-2 \frac{\left\{\nabla_{i} \nabla_{j} r\right\}}{r} \\
R_{i \alpha} & =0 \\
R_{\alpha \beta} & =\left\{\frac{2 m}{r}-r \nabla^{2} r\right\}^{F} g_{\alpha \beta}
\end{aligned}
$$

and for the Ricci scalar

$$
R={ }^{B} R-4 \frac{\nabla^{2} r}{r}+\frac{4 m}{r^{3}}
$$

where the Laplacians above are in the $(1+1)$ dimensional sense on the base space. The Einstein tensor takes the form

$$
\begin{aligned}
G_{i j} & =-\frac{2\left\{\nabla_{i} \nabla_{j} r\right\}}{r}+\left\{\frac{2 \nabla^{2} r}{r}-\frac{2 m}{r^{3}}\right\}{ }^{B} g_{i j} ; \\
G_{i \alpha} & =0 ; \\
G_{\alpha \beta} & =\left\{-\frac{{ }^{B} R r^{2}}{2}+r \nabla^{2} r\right\}{ }^{F} g_{\alpha \beta} .
\end{aligned}
$$

These are all purely geometrical statements - while one has chosen coordinates $x^{a}=\left(x^{i} ; x^{\alpha}\right)$ to make the warped product structure manifest, these results are completely independent of one's choice of coordinates $x^{i}$ on the radial-temporal plane (the base space), and for that matter are completely independent of one's choice of coordinates $x^{\alpha}$ on the spherically symmetric fibres. 


\subsection{The Kodama miracle}

It is well known that in a time-dependent spacetime, there is no (asymptotically timelike) Killing vector to define a preferred time coordinate. The calculation of important quantities such as the four-acceleration and the surface gravity become much more ambiguous. Additionally, there is no general consensus on the "best" form of the metric, nor on the "best" choice of the coordinate system.

An interesting insight on this problem is given by Kodama [1], who proved the existence of a divergence-free vector field for any time-dependent spherically symmetric metric. The Kodama vector, $k^{a}$, lies in the $(1+1)$ dimensional radial-temporal plane, so that $k^{a}=\left(k^{i} ; 0,0\right)$. More precisely

$$
k^{a}=\epsilon_{\perp}^{a b} \nabla_{b} r .
$$

where the tensor $\epsilon_{\perp}^{a b}$ is the $(1+1)$ dimensional Levi-Civita tensor in the radial-temporal plane, denoted $\epsilon_{\perp}^{i j}$, canonically embedded into $(3+1)$ dimensions according to the prescription

$$
\epsilon_{\perp}^{a b}=\left[\begin{array}{c|c}
\epsilon_{\perp}^{i j} & 0 \\
\hline 0 & 0
\end{array}\right] .
$$

It is straightforward to check that $k^{a} \nabla_{a} r=0$. Furthermore, if we define a positive semi-definite norm by $\|k\|^{2}=|g(k, k)|=\left|g^{-1}\left(k^{b}, k^{b}\right)\right|$, then $\|k\|=\|\nabla r\|$. (We shall use the superscripted symbol $b$ to denote the process of turning a vector into a covector by "lowering the index", and use the superscripted symbol $\sharp$ to denote the inverse process of turning a covector into a vector by "raising the index".) By appropriate choice of orientation on the radial-temporal plane one can choose $k^{a}$ to be (asymptotically) future pointing. It can also be defined by the more abstract statement

$$
k=\left(*_{2} \mathrm{~d} r\right)^{\sharp},
$$


where by this one means "calculate the one-form $\mathrm{d} r$, apply the $(1+1) \mathrm{di}$ mensional radial-temporal Hodge star operation, and use the metric to convert the resulting one-form to a contravariant vector". At this point it is necessary to emphasise, as originally pointed out by Kodama himself, that the Kodama vector does not in general reduce to the Killing vector in a static spacetime; all that one can say in general is that in static spacetimes it is parallel to the Killing vector. In regions where the Kodama vector is timelike (and we shall [informally at this stage] refer to this as the black hole exterior region, i.e., the domain of outer communication) the Kodama vector defines a preferred class of fiducial observers (FIDOs) [25] specified by the unit timelike 4 -vector

$$
V=\frac{k}{\|k\|}
$$

Although the Kodama vector provides a preferred "time direction", and simplifies the evolution equations of a dynamical spherically symmetric system [26, 27], it does not at this stage define a preferred "time coordinate". We shall subsequently use the Kodama vector plus the Clebsch decomposition theorem to construct a geometrically natural preferred time coordinate. To prove that the Kodama vector is divergence free the best strategy (with hindsight) is to consider the quantity

$$
\begin{aligned}
\nabla_{a}\left(\epsilon_{\perp}^{a b} / r^{2}\right) & =\frac{1}{\sqrt{-g_{4}}} \partial_{a}\left(\sqrt{-g_{4}} \epsilon_{\perp}^{a b} / r^{2}\right) \\
& =\frac{1}{r^{2} \sqrt{-g_{2}}} \partial_{a}\left(\sqrt{-g_{2}}\left[\begin{array}{c|c}
\epsilon_{\perp}^{i j} & 0 \\
\hline 0 & 0
\end{array}\right]\right) \\
& =\left(\frac{1}{r^{2} \sqrt{-g_{2}}} \partial_{i}\left[\sqrt{-g_{2}} \epsilon_{\perp}^{i j}\right] ; 0,0\right) \\
& =\frac{1}{r^{2}}\left({ }^{B} \nabla_{i} \epsilon_{\perp}^{i j} ; 0,0\right) \\
& =0 .
\end{aligned}
$$

Note that the last covariant derivative is a base space covariant derivative which vanishes since the $(1+1)$ dimensional Levi-Civiata tensor is covari- 
antly constant with respect to the $(1+1)$ dimensional covariant derivative. But this $(3+1)$ dimensional result, $\nabla_{a}\left(\epsilon_{\perp}^{a b} / r^{2}\right)=0$ can easily be rearranged to give

$$
k^{a}=\frac{r}{2} \nabla_{b} \epsilon_{\perp}^{a b}
$$

which now implies

$$
\nabla_{a} k^{a}=0
$$

so the Kodama vector itself is conserved. In addition, Kodama also proved that in time-dependent spherically symmetric spacetimes there is another (somewhat unexpected) conserved current. In terms of the Einstein tensor and the Kodama vector we have $J^{a}=G^{a b} k_{b}$, with:

$$
\nabla_{a} J^{a}=\nabla_{a}\left(G^{a b} k_{b}\right)=0
$$

This is a purely geometrical statement, ultimately due to the warped product form of the metric - it is not related to the Bianchi identities. Let us specifically compute

$$
J^{a}=G^{a b} k_{b}=\left(G^{i j} k_{j} ; 0,0\right)
$$

Now working on the radial-temporal base space we have

$$
G_{i j} k^{j}=-2 \frac{\nabla_{i} \nabla_{j} r}{r} \epsilon_{\perp}^{j k} \nabla_{k} r+\left\{\frac{2 \nabla^{2} r}{r}-\frac{2 m}{r^{3}}\right\} k^{i}
$$

But using the fact that in $(1+1)$ dimensions

$$
\nabla_{[i} r \epsilon_{\perp j k]}=0
$$

we have

$$
\begin{aligned}
\nabla_{i} \nabla_{j} r \epsilon_{\perp}^{j k} \nabla_{k} r & =\nabla^{j} \nabla_{i} r \epsilon_{\perp j k} \nabla^{k} r \\
& =\nabla^{j}\left\{\nabla_{i} r \epsilon_{\perp j k}\right\} \nabla^{k} r \\
& =\nabla^{j}\left\{-\nabla_{j} r \epsilon_{\perp k i}-\nabla_{k} r \epsilon_{\perp i j}\right\} \nabla^{k} r \\
& =+\nabla^{2} r k_{i}-\frac{1}{2} \epsilon_{\perp i j} \nabla^{j}\left\{|\nabla r|^{2}\right\} .
\end{aligned}
$$


Combining these results

$$
G_{i j} k^{j}=+\frac{1}{r} \epsilon_{\perp i j} \nabla^{j}\left\{|\nabla r|^{2}\right\}-\frac{2 m}{r^{3}} k^{i}
$$

But in view of the definition of the Hawking-Israel/ Hernandez-Misner/ Misner-Sharp quasi-local mass [23, 24] we then have

$$
G_{i j} k^{j}=-\frac{1}{r} \epsilon_{\perp i j} \nabla^{j}\{2 m / r\}-\frac{2 m}{r^{3}} k^{i} .
$$

That is

$$
G_{i j} k^{j}=-\frac{2}{r^{2}} \epsilon_{\perp i j} \nabla^{j} m
$$

This computation has been performed using the $(1+1)$ dimensional covariant derivative in the radial-temporal base space, but at this stage we can safely use the symmetries of the situation to lift this equality to the full spacetime

$$
J^{a}=G^{a b} k_{b}=-\frac{2}{r^{2}} \epsilon_{\perp}^{a b} \nabla_{b} m
$$

This is a purely geometrical statement - fundamentally connected with the warped product nature of the spacetime - that underlies the unexpected conservation of the Kodama current. In view of the fact that we have already proven $\nabla_{a}\left(\epsilon_{\perp}^{a b} / r^{2}\right)=0$ we finally see

$$
\nabla_{a} J^{a}=-\frac{2}{r^{2}} \epsilon_{\perp}^{a b} \nabla_{a} \nabla_{b} m=0 .
$$

Thus conservation of the Kodama flux is a subtle result deeply connected with the warped product nature of the spacetime. We have presented this derivation in some detail because it is now possible to rapidly generalize the result in a significant manner.

\subsection{Generalized Kodama flux}

Consider an arbitrary function $\chi(m, r)$ of the two quantities $m\left(x^{i}\right)$ and $r\left(x^{i}\right)$. Now construct the current

$$
J_{\chi}^{a}=\left\{\partial_{m} \chi(m, r) G^{a b}-2 \partial_{r} \chi(m, r) g^{a b}\right\} k_{a} .
$$


This current is conserved in any spherically symmetric spacetime. To prove this note that by the definition of the Kodama vector and the geometrical identity proved above we have

$$
\begin{aligned}
J_{\chi}^{a} & =-2 \frac{\epsilon_{\perp}^{a b}}{r^{2}}\left\{\partial_{m} \chi(m, r) \nabla_{b} m+\partial_{r} \chi(m, r) \nabla_{b} r\right\} \\
& =-2 \frac{\epsilon_{\perp}^{a b}}{r^{2}} \nabla_{b} \chi(m, r) .
\end{aligned}
$$

Conservation of this 4 -vector is then obvious from the last expression. Note in particular that by the above argument any flux of the form

$$
J_{12}^{a}=\left\{f_{1}(m) G^{a b}+f_{2}(r) g^{a b}\right\} k_{a}
$$

[for arbitrary $f_{1}(m)$ and $f_{2}(r)$ ] will automatically be conserved.

Formally, there is an even more general conserved current one can write down: For any arbitrary scalar function $\Psi\left(x^{i}\right)$ defined on the radialtemporal base space the quantity

$$
J_{\Psi}^{a}=\frac{\epsilon_{\perp}^{a b}}{r^{2}} \nabla_{b} \Psi
$$

is conserved. Though this result is more general, it is somewhat less geometrical, and does not have the same flavour as the above. If (and only if) the functions $r\left(x^{i}\right)$ and $m\left(x^{i}\right)$ are functionally independent (so that one can use $m$ and $r$ as coordinates on the radial-temporal base space) then these two notions $\left(J_{\chi}\right.$ and $\left.J_{\Psi}\right)$ can be made to coincide. In particular, $\Psi \rightarrow-2 m(r, t)$ gives us Kodama's conserved flux $J^{a}$, while $\Psi \rightarrow \frac{1}{3} r^{3}$ is just the statement that the Kodama vector itself is conserved, $\nabla_{a} k^{a}=$ 0 . (For related comments in a higher-dimensional Gauss-Bonnet context see [17, 18].)

\subsection{Kodama time}

The Kodama vector has been used before in several aspects of the timedependent gravitational collapse problem. However it has not been used 
to obtain a preferred time coordinate, nor a preferred coordinate system for the metric of a dynamic spacetime. Fortunately, in (1+1) dimensions it is possible to use the lesser known but classic Clebsch decomposition theorem, a result complementary to the more usual Helmholtz decomposition theorem, (see for instance [2, 3, 4]) to assert that there are two unique scalars $\alpha$ and $\beta$ such that the Kodama covector $k^{b}$ takes the form

$$
k^{b}=\alpha \mathrm{d} \beta .
$$

This decomposition is unique up to trivial transformations of the form $\beta \rightarrow$ $\bar{\beta}=f(\beta)$. Now in the "normal" exterior region where $\mathrm{d} r$ is spacelike, (i.e., in the domain of outer communication), the Kodama vector and covector are both timelike, so in this region the one-form $\mathrm{d} \beta$ is guaranteed to be timelike. This very strongly suggests that $\beta$ should be adopted as a preferred "time coordinate". In fact, relabeling the scalar $\beta$ as a coordinate $\beta \rightarrow t$, and relabeling the integrating factor as $\alpha \rightarrow F$, we write

$$
k^{b}=F \mathrm{~d} t
$$

This naturally induces a geometrically preferred time coordinate $t$ which we shall refer to as the Kodama time. (Note that this Kodama time is unique up to transformations of the form $t \rightarrow \bar{t}=f(t)$, which amount to simple relabeling of the constant-time slices, without any "mixing" between different constant-time slices.) Using this time coordinate is at least as natural as using $r$ for the radial coordinate. (This key step, though mathematically elementary, goes well beyond anything in Kodama's original paper [1], or the various papers that have subsequently sought to use Kodama's formalism.) As we shall soon see, this choice of Kodama time coordinate is the unique choice that makes integral curves of the vector $\partial_{t}$ coincide with integral curves of the Kodama vector. That is

$$
k \propto \partial_{t}
$$

Ultimately, adopting these coordinates (no matter how natural they appear) is of course a choice, and will be "justified" only insofar as they turn 
out to be useful.

Adopting these $(t, r)$ coordinates as preferred coordinates on the radialtemporal plane, and without any loss of generality, the metric can be written as

$$
\begin{array}{r}
\mathrm{d} s^{2}=g_{t t}(r, t) \mathrm{d} t^{2}+2 g_{t r}(r, t) \mathrm{d} r \mathrm{~d} t+g_{r r}(r, t) \mathrm{d} r^{2} \\
+r^{2}\left\{\mathrm{~d} \theta^{2}+\sin ^{2} \theta \mathrm{d} \phi^{2}\right\} .
\end{array}
$$

However, since the Kodama vector $k$ is orthogonal to $\mathrm{d} r$, then also $\mathrm{d} t$ is orthogonal to $\mathrm{d} r$, and so the cross term in the metric is zero. Thus in these preferred coordinates the metric is diagonal,

$$
\mathrm{d} s^{2}=g_{t t}(r, t) \mathrm{d} t^{2}+g_{r r}(r, t) \mathrm{d} r^{2}+r^{2}\left\{\mathrm{~d} \theta^{2}+\sin ^{2} \theta \mathrm{d} \phi^{2}\right\}
$$

This relatively long argument has ultimately led us back to one of the simplest, and arguably most obvious, forms of the metric - a simple diagonal metric in Schwarzschild curvature coordinates. (Of course now we can argue that we have a geometrically natural reason for adopting this particular set of coordinates.)

When using the Schwarzschild radial coordinate $r$ it is natural to choose the parameterization

$$
g_{r r}(r, t)=\left(1-\frac{2 m(r, t)}{r}\right)^{-1}
$$

for the radial-radial part of the metric tensor. Doing so will automatically give the quantity $m(r, t)$ a natural interpretation in terms of the HawkingIsrael/ Hernandez-Misner/ Misner-Sharp quasi-local mass [23, 24]. Since the radial-temporal plane by definition has Lorentzian signature, this choice then guarantees that it is possible to write the temporal-temporal component of the metric tensor in the form

$$
g_{t t}(r, t)=-e^{-2 \Phi(r, t)}\left(1-\frac{2 m(r, t)}{r}\right) .
$$


We finally have the (quite standard) result

$$
\begin{aligned}
\mathrm{d} s^{2}=-e^{-2 \Phi(r, t)} & \left(1-\frac{2 m(r, t)}{r}\right) \mathrm{d} t^{2}+\frac{\mathrm{d} r^{2}}{1-2 m(r, t) / r} \\
& +r^{2}\left\{\mathrm{~d} \theta^{2}+\sin ^{2} \theta \mathrm{d} \phi^{2}\right\},
\end{aligned}
$$

where in addition we know

$$
\nabla_{a} r=(\mathrm{d} r)_{a}=(0,1 ; 0,0)
$$

and the equivalent contravariant result

$$
(\mathrm{d} r)^{a}=\left(0,1-\frac{2 m(r, t)}{r} ; 0,0\right) .
$$

Furthermore, the components of the Kodama vector and covector in these coordinates are

$$
\begin{aligned}
& k^{a}=e^{\Phi(r, t)}(1,0 ; 0,0) \\
& k_{a}=-e^{-\Phi(r, t)}\left(1-\frac{2 m(r, t)}{r}\right)(1,0 ; 0,0) .
\end{aligned}
$$

As previously mentioned, the squared norm of the Kodama vector is equal to that of $\nabla r$ :

$$
\|k\|^{2}=\|\nabla r\|^{2}=\left|1-\frac{2 m(r, t)}{r}\right| .
$$

In these coordinates it is useful to define the time translation vector, $\mathcal{T}$, which is not a Killing vector unless the geometry happens to be static, as

$$
\begin{gathered}
\mathcal{T}=\partial_{t} ; \quad \mathcal{T}^{a}=(1,0 ; 0,0) \\
\mathcal{T}_{a}=e^{-2 \Phi(r, t)}\left(1-\frac{2 m(r, t)}{r}\right)(1,0 ; 0,0) .
\end{gathered}
$$

The squared norm of $\mathcal{T}$ is equal to the absolute value of the temporaltemporal component of the metric, $\left|g_{t t}\right|$, and proportional to the squared norm of the Kodama vector:

$$
\|\mathcal{T}\|^{2}=\left|g_{t t}\right|=e^{-2 \Phi(r, t)}\left|1-\frac{2 m(r, t)}{r}\right|=e^{-2 \Phi(r, t)}\|k\|^{2} .
$$


That is,

$$
e^{-2 \Phi(r, t)}=\frac{\|\mathcal{T}\|^{2}}{\|k\|^{2}} ; \quad e^{-\Phi(r, t)}=\frac{\|\mathcal{T}\|}{\|k\|}
$$

In the static situation it is the time translation vector $\mathcal{T}$ that will reduce to the (asymptotic) timelike Killing vector: $\mathcal{T} \rightarrow K \neq k$. Because the normalizations of $\mathcal{T}$ and $k$ differ, then if the Kodama vector is used simply as a substitute for the Killing vector when attempting to calculate quantities such as the surface gravity [5, 6, 7, 8], one is likely to encounter normalization issues when taking the static limit.

Specifically, to obtain a finite value of the four-acceleration near a possible horizon, as measured by an observer at infinity, it is necessary to multiply by a suitably defined normalizing factor [28, 29]. In the static case this normalizing factor is just $\left|g_{t t}\right|$, and coincides with the squared norm of the Killing vector. However, in the time dependent case, not only does the geometry not possess a Killing vector, but also the squared norm of the Kodama vector does not coincide with $\left|g_{t t}\right|$. This leaves us with a somewhat ambiguous situation with respect to the normalizing factor and the surface gravity of a time-dependent metric tensor, and means that we will have to exercise some care in defining the surface gravity of a timedependent geometry.

\subsection{Riemann tensor}

It is now a standard exercise to calculate the various components of the Riemann tensor (for instance, by using Maple). We note that the Riemann tensor is considerably less fearsome than one might suppose. Only one component is in any sense "difficult". Temporarily suppressing the $(r, t)$ 
arguments for conciseness, and working in an orthonormal basis we have:

$$
\begin{aligned}
R_{\hat{t} \hat{r} \hat{r} \hat{r}}= & -\frac{2 m}{r^{3}}-\frac{m^{\prime \prime}}{r}+\frac{2 m^{\prime}}{r^{2}} \\
& +\left(1-\frac{2 m}{r}\right)\left[-\Phi^{\prime \prime}+\left(\Phi^{\prime}\right)^{2}\right]+3(m / r)^{\prime} \Phi^{\prime} \\
& -\frac{e^{\Phi}}{r} \partial_{t}\left[\frac{\dot{m} e^{\Phi}}{\left(1-\frac{2 m}{r}\right)^{2}}\right] .
\end{aligned}
$$

In view of the warped product formalism, we know that this rather messy quantity has a direct and simple physical/ mathematical interpretation: As may be verified by direct computation it is simply ${ }^{B} R / 2$, one half the Ricci scalar of the $(1+1)$ dimensional radial-temporal plane.

The remaining components are much simpler:

$$
\begin{gathered}
R_{\hat{t} \hat{\theta} \hat{t} \hat{\theta}}=R_{\hat{t} \hat{\phi} \hat{t} \hat{\phi}}=\frac{m-r m^{\prime}}{r^{3}}-\left(1-\frac{2 m}{r}\right) \frac{\Phi^{\prime}}{r} \\
R_{\hat{t} \hat{\theta} \hat{r} \hat{\theta}}=R_{\hat{t} \hat{\phi} \hat{\phi} \hat{\phi}}=\frac{\dot{m} e^{\Phi}}{r^{2}(1-2 m / r)} ; \\
R_{\hat{r} \hat{\theta} \hat{r} \hat{\theta}}=R_{\hat{r} \hat{\phi} \hat{r} \hat{\phi}}=-\frac{m-r m^{\prime}}{r^{3}} .
\end{gathered}
$$

These three quantities are easily seen to be proportional to $\nabla_{i} \nabla_{j} r$. (In fact they equal $-\left\{\nabla_{\hat{i}} \nabla_{\hat{j}} r\right\} / r$.) Finally

$$
R_{\hat{\theta} \hat{\phi} \hat{\theta} \hat{\phi}}=\frac{2 m}{r^{3}} .
$$

Note that the particularly simple formula for $R_{\hat{\theta} \hat{\phi} \hat{\theta} \hat{\phi}}$ underlies the identification of $m(r, t)$ as the Hernandez-Misner quasi-local mass [23].

\subsection{Einstein tensor}

For the Einstein tensor, working in an orthonormal basis, the single most important result is

$$
G_{\hat{t} \hat{t}}=\frac{2 m^{\prime}(r, t)}{r^{2}}
$$


Here the primes denote differentiation with respect to $r$, and the dots with respect to $t$. This result is utterly standard, with the only novelty being that this formula for $G_{\hat{t} \hat{t}}$ continues to hold in the time dependent case (subject of course to the coordinate choices made above). This result for $G_{\hat{t} \hat{t}}$ is intimately related to the physical interpretation of $m(r, t)$ as the HawkingIsrael quasi-local mass.

A second important result is more subtle:

$$
G_{\hat{t} \hat{r}}=\frac{2 \dot{m}(r, t) e^{\Phi(r, t)}}{r^{2}\left(1-\frac{2 m(r, t)}{r}\right)}
$$

We shall soon see that this formula for $G_{\hat{t} \hat{r}}$ is central to the coordinatebased verification of Kodama's unexpected conservation law, and that it is intimately related to the Brown-York quasi-local mass [30].

For completeness we indicate

$$
G_{\hat{r} \hat{r}}=-\frac{2 m^{\prime}(r, t)}{r^{2}}-\frac{2 \Phi^{\prime}(r, t)\left(1-\frac{2 m(r, t)}{r}\right)}{r} .
$$

This result is quite standard, (see for instance equation (2.65) of [31]), with the only novelty being that this formula for $G_{\hat{r} \hat{r}}$ continues to hold in the time dependent case. This now implies the useful result

$$
G_{\hat{t} \hat{t}}+G_{\hat{r} \hat{r}}=-\frac{2 \Phi^{\prime}(r, t)\left(1-\frac{2 m(r, t)}{r}\right)}{r} .
$$

Finally, now suppressing the $(r, t)$ arguments for conciseness, we have

$$
\begin{aligned}
G_{\hat{\theta} \hat{\theta}}=G_{\hat{\phi} \hat{\phi}}= & \left(1-\frac{2 m}{r}\right)\left[-\Phi^{\prime \prime}+\Phi^{\prime}\left(\Phi^{\prime}-\frac{1}{r}\right)\right] \\
& -3 \Phi^{\prime}\left(\frac{m}{r^{2}}-\frac{m^{\prime}}{r}\right)-\frac{m^{\prime \prime}}{r} \\
& -\frac{e^{2 \Phi}}{\left(1-\frac{2 m}{r}\right)^{2}}\left[\frac{\ddot{m}}{r}+\frac{4(\dot{m})^{2}}{r\left(1-\frac{2 m}{r}\right)}-\frac{\dot{\Phi} \dot{m}}{r}\right] .
\end{aligned}
$$


The first two lines here are again quite standard, and appear also in static situations. (See for instance equation (2.66) of [31]). All the time derivatives have been isolated in the third line. With a little more work this can be somewhat tidied up as follows

$$
\begin{aligned}
G_{\hat{\theta} \hat{\theta}}= & G_{\hat{\phi} \hat{\phi}}=-\frac{m^{\prime \prime}}{r} \\
& -\frac{e^{\Phi}}{r \sqrt{1-2 m / r}} \partial_{r}\left[r\left(1-\frac{2 m}{r}\right)^{3 / 2} e^{-\Phi} \Phi^{\prime}\right] \\
& -\frac{e^{\Phi}}{r} \partial_{t}\left[\frac{\dot{m} e^{\Phi}}{\left(1-\frac{2 m}{r}\right)^{2}}\right] .
\end{aligned}
$$

This rather complicated expression can be verified to equal $-{ }^{B} R / 2+\nabla^{2} r / r$, the result we expect based on the warped product formalism. We note that the time derivative contributions to the Einstein tensor are quite isolated, and in this geometrically preferred coordinate system occur only in the $G_{\hat{\theta} \hat{\theta}}=G_{\hat{\phi} \hat{\phi}}$ and $G_{\hat{t} \hat{r}}$ components. This is ultimately one of the key reasons we will find the Kodama time to be so useful.

\subsection{Co-ordinate based version of Kodama's con- servation law}

Kodama's conservation law can now be studied in more explicit coordinatebased detail. First, based only on spherical symmetry and the definition of the Kodama vector, the unexpected conserved current $J^{a}$ takes the form

$$
J^{a}=g^{a b} G_{b c} k^{c}=\left\{-\hat{k}^{a} \hat{k}^{b}+(\widehat{\mathrm{d} r})^{a}(\widehat{\mathrm{d} r})^{b}\right\} G_{b c} \hat{k}^{c}\|k\|,
$$

whence, since by construction $\|k\|=\|\mathrm{d} r\|$, we see

$$
J^{a}=-G_{\hat{t} \hat{t}} k^{a}+G_{\hat{t} \hat{r}}(\mathrm{~d} r)^{a} .
$$


But we have already explicitly calculated the quantities $G_{\hat{t} \hat{t}}, G_{\hat{t} \hat{r}}, k^{a}$ and $(\mathrm{d} r)^{a}$. We obtain

$$
J^{a}=2\left(-\frac{e^{\Phi(r, t)} m^{\prime}(r, t)}{r^{2}}, \frac{e^{\Phi(r, t)} \dot{m}(r, t)}{r^{2}} ; 0,0\right)
$$

This vector is now obviously conserved since the 4-divergence is simply

$$
\begin{aligned}
\nabla_{a} J^{a} & =\frac{1}{\sqrt{-g_{4}}} \partial_{a}\left[\sqrt{-g_{4}} J^{a}\right] \\
& =\frac{2}{e^{-\Phi(r, t)} r^{2}} \partial_{a}\left[\left(-m^{\prime}(r, t), \dot{m}(r, t) ; 0,0\right)^{a}\right] \\
& =\frac{2}{e^{-\Phi(r, t)} r^{2}}\left[-\dot{m}^{\prime}(r, t)+\dot{m}^{\prime}(r, t)\right] \\
& =0 .
\end{aligned}
$$

Equivalently we note that from this coordinate-based calculation we explicitly recover

$$
J^{a}=G^{a b} k_{b}=-2 \frac{\epsilon_{\perp}^{a b} \nabla_{b} m}{r^{2}} .
$$

This relation is somewhat miraculous in the present coordinate based calculation, and as we have seen has a deeper justification in terms of the warped product form of the spacetime geometry.

\subsection{Brown-York quasi-local mass}

With the coordinate system developed above, the notion of quasi-local internal energy arises naturally as the Brown-York quasi-local mass [30]. To prove this, first let us take some imaginary spherical surface $r=r_{0}$, and hold $r_{0}$ fixed in time. Then the total energy inside this spherical surface depends on the net flux across the surface. To calculate the net flux we need the $G_{t r}$ component of the Einstein tensor, in an orthonormal basis. That is

$$
G_{\hat{t} \hat{r}}(r, t)=\frac{2 \dot{m}(r, t)}{r^{2}(1-2 m(r, t) / r)} e^{\Phi(r, t)}
$$


whence, via the Einstein equations $8 \pi G_{a b}=T_{a b}$, we have the flux density

$$
f(r, t)=T_{\hat{\hat{r}} \hat{r}}(r, t)=\frac{1}{4 \pi} \frac{\dot{m}(r, t)}{r^{2}(1-2 m(r, t) / r)} e^{\Phi(r, t)} .
$$

Now the total net flux across the imaginary surface at $r=r_{0}$, in an amount of proper time $\tau$, is

$$
\begin{aligned}
(\text { net flux })_{t_{\text {initial }}}^{t_{\text {inal }}}= & \int f\left(r_{0}, t\right) \times(\text { area }) \times \mathrm{d} \tau \\
= & \int f\left(r_{0}, t\right) \times\left(4 \pi r_{0}^{2}\right) \\
& \times\left[e^{-\Phi\left(r_{0}, t\right)} \sqrt{1-\frac{2 m\left(r_{0}, t\right)}{r_{0}}}\right] \times \mathrm{d} t .
\end{aligned}
$$

That is:

$$
\begin{aligned}
(\text { net flux })_{t_{\text {initial }}}^{t_{\text {final }}} & =\int \frac{\dot{m}\left(r_{0}, t\right)}{\sqrt{1-\frac{2 m\left(r_{0}, t\right)}{r}}} \mathrm{~d} t \\
& =\left[-r_{0} \sqrt{1-\frac{2 m\left(r_{0}, t\right)}{r_{0}}}\right]_{t_{\text {initial }}}^{t_{\text {final }}} .
\end{aligned}
$$

Then, if initially there is no mass inside $r=r_{0}$, at time $t_{\text {final }}$ we have

$$
(\text { net flux })_{t_{\text {initial }}}^{t_{\text {final }}}=r_{0}\left(1-\sqrt{1-\frac{2 m\left(r_{0}, t_{\text {final }}\right)}{r_{0}}}\right) .
$$

In this situation, the only meaningful definition of internal energy at $t=$ $t_{\text {initial }}$ is to set $U\left(r_{0}, t_{\text {initial }}\right)=0$. Hence, at any subsequent time $t$ the internal energy $U\left(r_{0}, t\right)$ is equal to the net incoming flux and so it makes sense to define

$$
U\left(r_{0}, t\right)=r_{0}\left(1-\sqrt{1-\frac{2 m\left(r_{0}, t\right)}{r_{0}}}\right) .
$$


This internal energy is just the Brown-York quasi-local mass for the spacetime geometry with metric (2.52), see [30]. We can rearrange this (as pointed out in [30]) to yield

$$
m\left(r_{0}, t\right)=U\left(r_{0}, t\right)-\frac{U^{2}\left(r_{0}, t\right)}{2 r_{0}} .
$$

Here $m\left(r_{0}, t\right)$ retains its interpretation as the Hawking-Israel (and Hernandez-Misner/ Misner-Sharp [23, 24]) quasi-local mass. The difference between the two notions of energy is just the self interacting Newtonian gravitational potential of a massive shell of radius $r_{0}$. Both energies coincide at spatial infinity with the ADM mass.

\subsection{Surface gravity}

Several attempts at calculating the surface gravity for a time-dependent metric have been made using the Kodama vector instead of the Killing vector [5, 6, 7, 8], with results qualitatively similar to those in the static case; even to the extent of deriving some form of the first law of (black hole) thermodynamics.

\subsubsection{Surface gravity from fiducial observers}

The most intuitive way of calculating the surface gravity is by working in the exterior region and considering the four-velocity $V$ parallel to the Kodama vector. Calculate the four-acceleration $A=\nabla_{V} V$. Then explicitly computing the magnitude of this four-acceleration we see

$$
\begin{aligned}
a=\|A\|= & \frac{1}{\sqrt{1-\frac{2 m(r, t)}{r}}}\left[\frac{m(r, t)}{r^{2}}-\frac{m^{\prime}(r, t)}{r}\right] \\
& -\sqrt{1-\frac{2 m(r, t)}{r}} \Phi^{\prime}(r, t) .
\end{aligned}
$$

(Note that near spatial infinity we have the sensible Newtonian result $a \rightarrow m / r^{2}$.) The surface gravity can be defined as the acceleration of an 
observer near the evolving horizon, which we implicitly define by $r_{H}(t)=$ $2 m\left(r_{H}(t), t\right)$, as measured by another observer at infinity. Thus, at this point it is necessary to multiply by a normalizing factor, often referred to as a redshift factor. In the (asymptotically flat) static case there is no doubt that the appropriate normalizing factor is

$$
\|K\|=\left|g_{t t}\right|^{1 / 2}=e^{-\Phi(r)} \sqrt{1-2 m(r) / r}
$$

and that the appropriate object to consider is the near horizon limit of

$$
\begin{aligned}
\kappa_{\text {static }} & =\|A\|\|K\|=\left\|\nabla_{K} V\right\| \\
& =e^{-\Phi(r)}\left\{\left[\frac{m(r)}{r^{2}}-\frac{m^{\prime}(r)}{r}\right]-\left[1-\frac{2 m(r)}{r}\right] \Phi^{\prime}(r)\right\} .
\end{aligned}
$$

The location of the horizon is in the static case implicitly defined by $r_{H}=$ $2 m\left(r_{H}\right)$, and this is now a true Killing horizon and also an event horizon, at which we have the standard result [16]

$$
\kappa_{\text {static } \mid H}=e^{-\Phi\left(r_{H}\right)}\left\{\frac{1-2 m^{\prime}\left(r_{H}\right)}{2 r_{H}}\right\} .
$$

When bootstrapping this to the dynamic case a plausible generalization (which we shall subsequently buttress by also considering the radial null geodesics) is to replace $\|K\| \rightarrow\|\mathcal{T}\|$, which at least has the virtue of maintaining the correct static limit. Under this hypothesis the appropriate object to consider is the near horizon limit of

$$
\begin{aligned}
\kappa_{V} & =\|A\|\|\mathcal{T}\|=\left\|\nabla_{\mathcal{T}} V\right\| \\
& =e^{-\Phi(r, t)}\left\{\left[\frac{m(r, t)}{r^{2}}-\frac{m^{\prime}(r, t)}{r}\right]-\left[1-\frac{2 m(r, t)}{r}\right] \Phi^{\prime}(r, t)\right\} .
\end{aligned}
$$

which on the evolving horizon has the limit

$$
\kappa_{V \mid H}(t)=e^{-\Phi\left(r_{H}(t), t\right)}\left\{\frac{1-2 m^{\prime}\left(r_{H}(t), t\right)}{2 r_{H}(t)}\right\} .
$$

However, this proposed definition presents us with a potential ambiguity - what is the physically most appropriate choice for the normalizing 
factor? The choice of $\|\mathcal{T}\|$ as normalizing factor as advocated above is certainly plausible, and has the correct static limit. Furthermore it is intimately related to the Kodama time introduced previously, rather than the Kodama vector $k$. Nevertheless, it is useful to see if we can come up with other plausible candidates for surface gravity in an evolving spacetime, and see whether they agree with (or are closely related to) the above proposal, and whether they possess the correct static limit.

\subsubsection{Surface gravity from radial null geodesics}

There are other, possibly less ambiguous, ways to usefully define the sought after surface gravity. More specifically, we can parameterize the strength of the gravitational field throughout the entire spacetime geometry by using the inaffinity properties of the radial null geodesics. Consider (temporarily) the following null vectors:

$$
\tilde{\ell}_{a}^{ \pm}=\frac{ \pm k_{a}+\nabla_{a} r}{2}
$$

In the exterior region (where $k$ is timelike) these null vectors are both outward-pointing, $\tilde{\ell}^{+}$is future-pointing, and $\tilde{\ell}^{-}$is past-pointing. (Note that $-\tilde{\ell}^{-}$is then inward pointing; these specific conventions have been chosen to simplify the computations below as far as possible.) These are arguably the simplest radial null vectors one could write down using only the Kodama vector. It is easy to check that $\tilde{\ell}_{a}^{+} \tilde{\ell}_{-}^{a}=\frac{1}{2}\|k\|^{2}$. Since we are working with spherical symmetry, both radial null vectors must satisfy the geodesic equation (in its non-affine parameterized form):

$$
\tilde{\ell}_{ \pm}^{b} \nabla_{b} \tilde{\ell}_{ \pm}^{a}=\tilde{\kappa}_{\ell_{ \pm}} \tilde{\ell}_{ \pm}^{a} ; \quad \nabla_{\tilde{\ell}_{ \pm}} \tilde{\ell}_{ \pm}=\tilde{\kappa}_{\ell_{ \pm}} \tilde{\ell}_{ \pm} ;
$$

where $\tilde{\kappa}_{\ell \pm}$ are scalars defined everywhere throughout the spacetime. By contracting these equations with $\tilde{\ell}_{a}^{\mp}$, we can explicitly compute $\tilde{\kappa}_{\ell_{ \pm}}$, to yield:

$$
\tilde{\kappa}_{\ell_{+}}=\tilde{\kappa}_{\ell_{-}}=\frac{m(r, t)}{r^{2}}-\frac{m^{\prime}(r, t)}{r}-\frac{1}{2}\left[1-\frac{2 m(r, t)}{r}\right] \Phi^{\prime}(r, t) .
$$


(Note that near spatial infinity we have $\tilde{\kappa}_{\ell_{ \pm}} \rightarrow m / r^{2}$.) At the evolving horizon this would reduce to the tentative definition

$$
\tilde{\kappa}_{H}(t)=\frac{1-2 m^{\prime}\left(r_{H}(t), t\right)}{2 r_{H}(t)} .
$$

Unfortunately this does not reduce to the known result in the static case there is a missing factor of $e^{-\Phi\left(r_{H}(t), t\right)} \rightarrow e^{-\Phi\left(r_{H}\right)}$. This makes the above definition not suitable for calculating the Hawking temperature. (We emphasize that in static situations the standard Wick-rotation trick of going to Euclidean signature, demanding the absence of any conical singularity at $r_{H}$, and interpreting the Hawking temperature in terms of periodicity in imaginary time, uniquely enforces equation (2.86) as the only physically acceptable candidate for the surface gravity [16].) The source of the difficulty is, since $e^{-\Phi(r, t)}=\|\mathcal{T}\| /\|k\|$, ultimately due to the fact that $\|\mathcal{T}\| \neq\|k\|$ in general. These considerations do suggest an improved strategy: Since we have seen how to use the Clebsch decomposition to deduce the natural existence of a Kodama time, in addition to a Kodama vector, then it would seem appropriate to use the Kodama time as the natural (non-affine) parameter for these radial null curves. (That is, we now parameterize the null curves by Kodama time, rather than the usual Killing time used in the static case.) This is tantamount to choosing

$$
\ell_{a}^{ \pm}=\frac{1}{2}\left[ \pm \mathcal{T}_{a}+\frac{\|\mathcal{T}\|}{\|k\|} \nabla_{a} r\right]=e^{-\Phi(r, t)} \tilde{\ell}_{a}^{ \pm}
$$

This time, the inner product is $\ell_{a}^{+} \ell_{-}^{a}=\frac{1}{2}\|\mathcal{T}\|^{2}$. These "Kodama time normalized" radial null vectors are again tangent to the radial null geodesics and so satisfy

$$
\ell_{ \pm}^{b} \nabla_{b} \ell_{ \pm}^{a}=\kappa_{\ell_{ \pm}} \ell_{ \pm}^{a}
$$

A brief calculation yields

$$
\kappa_{\ell_{ \pm}}=e^{-\Phi(r, t)} \tilde{\kappa}_{\ell_{ \pm}}-\ell_{ \pm}^{a} \nabla_{a} \Phi
$$


whence

$$
\begin{aligned}
\kappa_{\ell_{ \pm}}=e^{-\Phi(r, t)} & \left\{\left[\frac{m(r, t)}{r^{2}}-\frac{m^{\prime}(r, t)}{r}\right]\right. \\
- & {\left.\left[1-\frac{2 m(r, t)}{r}\right] \Phi^{\prime}(r)\right\} \mp \frac{1}{2} \dot{\Phi}(r, t) . }
\end{aligned}
$$

At the evolving horizon, $\kappa_{\ell_{ \pm}}$reduces to

$$
\begin{gathered}
\kappa_{\ell_{ \pm} \mid H}(t)=e^{-\Phi\left(r_{H}(t), t\right)}\left\{\frac{1-2 m^{\prime}\left(r_{H}(t), t\right)}{2 r_{H}(t)}\right\} \\
\mp \frac{1}{2} \dot{\Phi}\left(r_{H}(t), t\right) .
\end{gathered}
$$

This is not quite equal to $\kappa_{V \mid H}$ - though it does share with $\kappa_{V \mid H}$ the desirable property of having the correct static limit. An improved proposal is to average over past and future pointing null geodesics and take

$$
\kappa_{\text {null }}=\frac{1}{2}\left[\kappa_{\ell_{+}}+\kappa_{\ell_{-}}\right]
$$

Then $\kappa_{\text {null }}=\kappa_{V}$. That is: If one takes future-pointing and past-pointing outward null geodesics, normalized to Kodama time, and averages the resulting inaffinity parameters, then one obtains the same $\kappa_{V}$ that we tentatively identified based on the 4-acceleration of the FIDOs that follow the Kodama flow.

In short: By using Kodama time in addition to the Kodama vector we have now developed a geometrically preferred notion of surface gravity for spherically symmetric evolving spacetimes that can meaningfully be extended throughout the entire spacetime, and in addition exhibits a good static limit.

\subsection{The evolving horizon}

With the calculations presented so far, it is not possible to conclude too much about the evolving horizon at $r_{H}(t)=2 m\left(t, r_{H}\right)$. To relate this to 
a trapping horizon, in the Hayward sense [5, 6, 7, 8, 9, 10, 11, 12, 13, 14], it is necessary to compute the expansions, $\theta_{\tilde{\ell}_{ \pm}}$and $\theta_{\ell_{ \pm}}$, of the radial null vectors. Let us use the following definitions for the expansion [15]

$$
\theta_{\tilde{\ell}_{ \pm}}=\nabla_{a} \tilde{\ell}_{ \pm}^{a}-\tilde{\kappa}_{\ell_{ \pm}} ; \quad \theta_{\ell_{ \pm}}=\nabla_{a} \ell_{ \pm}^{a}-\kappa_{\ell_{ \pm}} ;
$$

A brief computation yields

$$
\begin{aligned}
\theta_{\tilde{\ell}_{ \pm}} & =\frac{1}{r}\left(1-\frac{2 m(r, t)}{r}\right) \\
\theta_{\ell_{ \pm}} & =\frac{1}{r}\left(1-\frac{2 m(r, t)}{r}\right) e^{-\Phi(r, t)} .
\end{aligned}
$$

In particular both $\theta_{\tilde{\ell}_{+}}$and $\theta_{\ell_{+}}$change sign at the evolving horizon $r_{H}(t)$. This is sufficient to guarantee that the evolving horizon, which we see is at $r_{H}(t)=2 m\left(t, r_{H}(t)\right)$, is an apparent horizon. However $r_{H}(t)$ is not a trapping horizon in the Hayward sense [5, 6, 7, 8], nor an "Ashtekar horizon" [32, 33, 34], since in addition $\theta_{\tilde{\ell}_{-}}$and $\theta_{\ell_{-}}$also both vanish on the evolving horizon. This is not critical for our purposes, since ultimately a trapping horizon is not needed to have Hawking radiation [35, 36, 37].

For completeness, we have also computed $\tilde{\ell}_{-}^{a} \nabla_{a} \theta_{\tilde{\ell}_{+}}$and $\ell_{-}^{a} \nabla_{a} \theta_{\ell_{+}}$. At the evolving horizon we have

$$
\begin{aligned}
\left(\tilde{\ell}_{-}^{a} \nabla_{a} \theta_{\tilde{\ell}_{+}}\right)_{H} & =\frac{\dot{m}\left(r_{H}(t), t\right)}{r_{H}(t)^{2}} e^{\Phi\left(r_{H}(t), t\right)} \\
\left(\ell_{-}^{a} \nabla_{a} \theta_{\ell_{+}}\right)_{H} & =\frac{\dot{m}\left(r_{H}(t), t\right)}{r_{H}(t)^{2}} e^{-\Phi\left(r_{H}(t), t\right)} .
\end{aligned}
$$

Thus for both normalizations we have most (but not all) of the key properties of an outer trapping horizon at $r_{H}(t)$ when $\dot{m}\left(t, r_{H}(t)\right)>0$, i.e., when the overall mass increases in time.

\subsection{Discussion}

We have used the warped product formalism to investigate the geometry of time-dependent spherically symmetric spacetimes, developing relatively straightforward arguments for the covariant conservation of the 
Kodama vector itself and the associated Kodama flux. This construction has allowed us to construct a very general class of conserved fluxes appropriate to any spherically symmetric spacetime.

Furthermore, we have successfully used the Kodama vector field, plus the Clebsch decomposition, to obtain a preferred Kodama time coordinate, and have then proceeded to construct a geometrically preferred coordinate system for describing spherically symmetric time dependent spacetimes. The resulting metric is one of the most simple forms of the metric of a spherically symmetric spacetime - a diagonal metric in Schwarzchild curvature coordinates. With these coordinates there are very simple physical interpretations for both the Hawking-Israel (Hernandez-Misner/ MisnerSharp) and Brown-York quasi-local masses. Although the definition of surface gravity remains somewhat ambiguous, by using the Kodama time as an integral part of the construction we have identified some very good geometrically preferred candidates that are compatible with known results in the static limit.

As an attempt at generalizing the Kodama vector formulation to more general spacetimes (not only spherical symmetry), we could consider the so called dual mean curvature vector [38]. This vector is constructed via another geometrically preferred vector, the mean curvature vector. For any surface $\mathcal{S}$ embedded in spacetime $(M, g)$, the mean curvature vector is defined as the trace of the extrinsic curvature tensor (also known as the shape tensor). The mean curvature vector has been used extensively to study the nature of different trapped surfaces [39, 40, 41]. Once the mean curvature vector is defined, its dual is constructed by applying the 2 -dimensional Hodge star in the plane normal to the surface $\mathcal{S}$. (Thus this construction will work only if $\mathcal{S}$ is of co-dimension 2 in the manifold $M$.) The dual mean curvature vector will be automatically parallel to the Kodama vector in those situations where the Kodama vector is defined. Geometrically, the mean curvature vector lies in the direction in which the expansion of the surface $\mathcal{S}$ in spacetime is extremal [38]. For the surfaces 
of interest this direction is spacelike, hence the corresponding dual mean curvature vector will be timelike. It is within this framework that Kodamas conservation laws could also be studied, and we hope ultimately to generalize them beyond spherical symmetry. 



\section{3 \\ Entropy bounds}

—ntropy bounds in General Relativity usually represent a combination of classical and semi-classical aspects of the theory, however most of the bounds found in the literature rely heavily on the semi-classical, i.e., quantum field theory in curved spacetime. In this chapter we will develop a series of entropy bounds, for uncollapsed matter, that do not rely too much in the construction of a quantum field theory in curved spacetime.

First, using a generalization of the surface gravity, we build a purely classical bound on the Tolman mass of any static spacetime. Then we can invoke the Unruh effect to relate the generalized surface gravity to a temperature, and ultimately obtain a bound on the entropy of a static uncollapsed body. This bound is closely related to the usual entropy bounds, and although it is much more easy to derive, it is quite robust.

Second, we will consider a rotating uncollapsed blob of matter, this needs more detailed analysis than the simpler non-rotating scenario, including restrictions on the symmetries of the spacetime. Furthermore, unlike the non-rotating case, the Tolman mass is not the quantity to be bound. Nevertheless, by using one of the results of Chapter 4 we are still able to construct a classical bound on a related current. Then we again invoke the Unruh effect, to assert that the entropy of the body is also less or equal than one half of its total surface area. 


\subsection{Entropy bounds for static uncollapsed mat- ter}

\subsubsection{Tolman mass and generalized surface gravity}

Tolman mass is one of the standard notions of quasi-local mass in common use in general relativity [42]. Using classical general relativity, this quasilocal Tolman mass can, in any static spacetime (either with or without a black hole region), be reduced to a flux integral of (generalized) surface gravity across the boundary of the region of interest. (This is closely related to the classical laws of black hole mechanics [43].) General relativistic thermodynamics, together with a minimal appeal to quantum physics as embodied in the Unruh effect [44], is then sufficient to develop elementary but powerful bounds on a suitably defined notion of quasi-local entropy — bounds very similar in spirit to the holographic bound [45, 46, 47, 48, 49, 50], and closely related to entanglement entropy [51].

In a static spacetime where the metric is taken to be of the form

$$
\mathrm{d} s^{2}=-e^{-2 \Psi} \mathrm{d} t^{2}+g_{i j} \mathrm{~d} x^{i} \mathrm{~d} x^{j}
$$

the Tolman mass contained in a region $\Omega$ is defined in terms of the orthonormal components of stress-energy by first taking $\rho=T_{\hat{0} \hat{0}}$ and $p=$ $\frac{1}{3} \operatorname{tr}\left\{T_{\hat{\imath} \hat{\jmath}}\right\} ;$ and then setting

$$
m_{T}(\Omega)=\int_{\Omega} \sqrt{-g_{4}}\{\rho+3 p\} \mathrm{d}^{3} x .
$$

The Einstein equations then imply the purely geometrical statement

$$
m_{T}(\Omega)=\frac{1}{4 \pi} \int_{\Omega} \sqrt{-g_{4}} R_{\hat{0} \hat{0}} \mathrm{~d}^{3} x .
$$

The Tolman mass is intimately related to the Komar mass [52], though we will not be phrasing any of the discussion below in terms of Killing vectors. It is a very old result, going back at least as far as Landau-Lifshitz [53] 
that in any stationary metric

$$
R_{0}^{0}=\frac{1}{\sqrt{-g_{4}}} \partial_{i}\left(\sqrt{-g_{4}} g^{0 a} \Gamma_{a 0}^{i}\right) .
$$

(Here $a \in\{0,1,2,3\} ; i \in\{1,2,3\}$.) Adopting the manifest static coordinates of equation (3.1), and then going to an orthonormal basis, this is more simply phrased as

$$
R_{\hat{0} \hat{0}}=\frac{1}{\sqrt{-g_{4}}} \partial_{i}\left(\sqrt{-g_{4}}\left|g^{00}\right| \Gamma_{00}^{i}\right) .
$$

To get a clean physical interpretation of this formula, consider a fiducial observer (FIDO) with 4-velocity

$$
V^{a}=\left(\sqrt{\left|g^{00}\right|} ; 0,0,0\right) .
$$

By definition the 4-acceleration of these FIDOs is

$$
\begin{aligned}
A^{a} & =\left(\nabla_{V} V\right)^{a}=V^{b} \nabla_{b} V^{a}=V^{0}\left(\partial_{0} V^{a}+\Gamma^{a}{ }_{c 0} V^{c}\right) \\
& =\sqrt{\left|g^{00}\right|} \Gamma^{a}{ }_{00} \sqrt{\left|g^{00}\right|}=\left|g^{00}\right| \Gamma^{a}{ }_{00} .
\end{aligned}
$$

But then, since $V$ is 4-orthogonal to $A$, we have

$$
A^{0}=0 ; \quad A^{i}=\left|g^{00}\right| \Gamma^{i}{ }_{00} ;
$$

where $A^{i}$ are the 3 spatial components of 4 -acceleration. Therefore in any static spacetime, in the region outside the horizon, the Landau-Lifshitz result is

$$
R_{\hat{0} \hat{0}}=\frac{1}{\sqrt{-g_{4}}} \partial_{i}\left(\sqrt{-g_{4}} A^{i}\right) .
$$

Then for any 3-volume $\Omega$ (if a horizon is present then for convenience we confine ourselves to a region that lies outside the horizon) we can use ordinary partial derivative integration by parts to deduce

$$
\begin{aligned}
\int_{\Omega} \sqrt{-g_{4}} R_{\hat{0} \hat{o}} \mathrm{~d}^{3} x & =\int_{\Omega} \partial_{i}\left(\sqrt{-g_{4}} A^{i}\right) \mathrm{d}^{3} x \\
& =\int_{\Omega} \partial_{i}\left(\sqrt{g_{3}}\left\{e^{-\Psi} A^{i}\right\}\right) \mathrm{d}^{3} x \\
& =\int_{\partial \Omega}\left\{e^{-\Psi} A^{i}\right\} \hat{n}_{i} \sqrt{g_{2}} \mathrm{~d}^{2} x
\end{aligned}
$$


where $\hat{n}$ is the unit normal, (defined in terms of the 3-metric $g_{i j}$ ), and $\sqrt{g_{2}}$ is the induced area measure on $\partial \Omega$. Define a (generalized) surface gravity (3-vector) and its norm by

$$
\kappa^{i}=e^{-\Psi} A^{i} ; \quad \kappa=\sqrt{g_{i j} \kappa^{i} \kappa^{j}}=e^{-\Psi} \sqrt{g_{i j} A^{i} A^{j}} .
$$

This is just the "redshifted" 4-acceleration of the FIDOs, and is a natural generalization of surface gravity, not just for any event horizon that might be present, but also applying to FIDOs skimming along the boundary $\partial \Omega$. In terms of this generalized surface gravity we now have

$$
m_{T}(\Omega)=\frac{1}{4 \pi} \int_{\partial \Omega} \kappa^{i} \hat{n}_{i} \sqrt{g_{2}} \mathrm{~d}^{2} x=\frac{1}{4 \pi} \int_{\partial \Omega} \vec{\kappa} \cdot \hat{n} \mathrm{~d} \mathscr{A} .
$$

Defining an average surface gravity $\bar{\kappa}(\partial \Omega)$, a total area $\mathscr{A}(\partial \Omega)$, and temporarily assuming we have no black hole regions to deal with, we see

$$
m_{T}(\Omega) \leq \frac{1}{4 \pi} \int_{\partial \Omega} \kappa \mathrm{d}(\text { area })=\frac{\bar{\kappa}(\partial \Omega) \times \mathscr{A}(\partial \Omega)}{4 \pi} .
$$

So for any (static) horizonless object such as a star or planet, (or monster [54, 55, 56], or gravastar [57, 58, 59, 60], or black star [61, 62, 63, 64], or quasi-black hole [65, 66]), we can bound its total Tolman mass in terms quantities measurable on its surface:

$$
m_{T}(\Omega) \leq \frac{\bar{\kappa}(\partial \Omega) \times \mathscr{A}(\partial \Omega)}{4 \pi} .
$$

This gives us a very general bound on the Tolman mass.

\subsubsection{Entropy bounds and the Unruh effect}

Now introduce thermodynamics: Consider the Euler relation (essentially the Gibbs-Duhem relation) for the entropy density of matter - we are thinking of some equilibrium collection of atoms/molecules/fields making up a star/planet/monster/gravastar/black star/ quasi-black hole. (No event horizons for now.) Then

$$
s=\frac{\rho+p-\mu n}{T},
$$


where (as previously) $p=\frac{1}{3} \operatorname{tr}\left\{T_{\hat{\imath} \hat{\jmath}}\right\}$. Furthermore $\mu$ is the chemical potential and $n$ is the particle density. The total entropy due to matter inside any specified 3-volume is

$$
S(\Omega)=\int_{\Omega} \sqrt{g_{3}} s \mathrm{~d}^{3} x=\int_{\Omega} \sqrt{g_{3}} \frac{\rho+p-\mu n}{T} \mathrm{~d}^{3} x .
$$

But the Tolman equilibrium conditions are [42, 67, 68, 69]

$$
T \sqrt{-g_{00}}=T_{\infty} ; \quad \mu \sqrt{-g_{00}}=\mu_{\infty}
$$

where we assume asymptotic flatness and without loss of generality set $g_{00} \rightarrow 1$ at spatial infinity. Then

$$
S(\Omega)=\frac{1}{T_{\infty}} \int_{\Omega} \sqrt{-g_{4}}\{\rho+p\} \mathrm{d}^{3} x-\frac{\mu_{\infty}}{T_{\infty}} \int_{\Omega} \sqrt{g_{3}} n \mathrm{~d}^{3} x,
$$

that is

$$
S(\Omega)=\frac{1}{T_{\infty}} \int_{\Omega} \sqrt{-g_{4}}\{\rho+p\} \mathrm{d}^{3} x-\frac{\mu_{\infty} N}{T_{\infty}} .
$$

But thermodynamic stability requires $\mu \geq 0$, so

$$
S(\Omega) \leq \frac{1}{T_{\infty}} \int_{\Omega} \sqrt{-g_{4}}\{\rho+p\} \mathrm{d}^{3} x
$$

Furthermore, in any system such as a star or planet $p>0$ throughout the interior so we have

$$
S(\Omega) \leq \frac{1}{T_{\infty}} \int_{\Omega} \sqrt{-g_{4}}\{\rho+3 p\} \mathrm{d}^{3} x,
$$

which implies

$$
S(\Omega) \leq \frac{m_{T}(\Omega)}{T_{\infty}}
$$

That is - the entropy inside any equilibrium star/planet or monster/gravastar/black star/quasi-black hole is bounded by the Tolman mass divided by the temperature (normalized at infinity). By our theorem above

$$
S(\Omega) \leq \frac{\bar{\kappa}(\partial \Omega) \times \mathscr{A}(\partial \Omega)}{4 \pi T_{\infty}}
$$


where so far we have only used basic thermodynamics and no curved space quantum field theory.

Furthermore, due to the existence of the Unruh acceleration radiation phenomenon [44], we can argue that an observer at position $x$ on the boundary $\partial \Omega$ will see a minimum locally measured temperature of

$$
T(x) \geq T_{\operatorname{Unruh}}(x)=\frac{\|A(x)\|}{2 \pi},
$$

which when redshifted to infinity implies

$$
T_{\infty} \geq \max _{x \in \partial \Omega}\left\{\sqrt{-g_{00}(x)} T_{\text {Unruh }}(x)\right\}=\max _{x \in \partial \Omega}\left\{\frac{\kappa(x)}{2 \pi}\right\} .
$$

So the equilibrium temperature of a star/planet/ monster/gravastar/black star/quasi-black hole confined inside a boundary $\partial \Omega$ must satisfy

$$
T_{\infty} \geq \frac{\bar{\kappa}(\partial \Omega)}{2 \pi}
$$

So finally

$$
S(\Omega) \leq \frac{\mathscr{A}(\partial \Omega)}{2}
$$

That is: Under very mild conditions, and even with a number of suboptimal inequalities being used in the derivation, we have nevertheless been able to see that the total entropy of a star/planet/monster/gravastar/ black star/quasi-black hole is bounded by half its area - this is very close to the holographic bound [45], which corresponds to $S(\Omega) \leq \frac{1}{4} \mathscr{A}(\partial \Omega)$, and also seems closely related to the generalized second law [46] and to the Bekenstein bound $S(\Omega) \leq 2 \pi E(\Omega) R(\Omega)$ [47]. This is also similar in spirit to Srednicki's entanglement entropy [51] — we are bounding the entropy in terms of the visible surface $\partial \Omega$ without looking "inside" $\Omega$. Srednicki's entanglement entropy argument would yield $S(\Omega) \propto \mathscr{A}(\partial \Omega)$ with an unknown and cutoff-dependent proportionality constant. While our argument provides a precise numerical value for the proportionality constant, $\frac{1}{2}$, unfortunately we have not yet been able to improve the proportionality constant to the $\frac{1}{4}$ one expects based on the holographic bound. On 
the other hand, the very mildness of the assumptions used in the bound makes it of some independent interest in its own right.

\subsubsection{Spherical symmetry}

As a consistency check consider a static spherically symmetric geometry. Without loss of generality choose coordinates to write the metric in the form

$$
\begin{aligned}
\mathrm{d} s^{2}= & -e^{-2 \Phi(r)}\left[1-\frac{2 m(r)}{r}\right] \mathrm{d} t^{2}+\frac{\mathrm{d} r^{2}}{1-2 m(r) / r} \\
& +r^{2}\left\{\mathrm{~d} \theta^{2}+\sin ^{2} \theta \mathrm{d} \phi^{2}\right\} .
\end{aligned}
$$

Note that with these conventions

$$
\sqrt{-g_{4}}=\sqrt{-g_{00}} \sqrt{g_{3}}=e^{-\Phi} r^{2} \sin \theta \rightarrow 4 \pi r^{2} e^{-\Phi}
$$

We implicitly assume asymptotic flatness, and normalize to $\Phi(\infty)=0$. The Killing horizon is defined by the location where $2 m(r) / r=1$, that is

$$
2 m\left(r_{H}\right)=r_{H}
$$

Then it is an old result [16] that at the horizon

$$
\kappa_{H}=\frac{e^{-\Phi_{H}}\left(1-2 m_{H}^{\prime}\right)}{2 r_{H}} .
$$

By looking at integral curves of the Killing vector, it is easy to see that the 4-acceleration of the FIDOs is

$$
A(r)=\left\{\frac{m(r)-r m^{\prime}(r)}{r^{2} \sqrt{1-2 m(r) / r}}-\Phi^{\prime}(r) \sqrt{1-\frac{2 m(r)}{r}}\right\} .
$$

A "red-shifted" normalized "generalized surface gravity" can now be defined for arbitrary $r$ by taking

$$
\kappa(r)=\sqrt{-g_{00}} A(r)=e^{-\Phi(r)} \sqrt{1-2 m(r) / r} A(r),
$$


so

$$
\kappa(r)=e^{-\Phi(r)}\left\{\frac{m(r)-r m^{\prime}(r)}{r^{2}}-\Phi^{\prime}(r)\left[1-\frac{2 m(r)}{r}\right]\right\} .
$$

Note that this is now not the surface gravity of the black hole region, but rather the surface gravity of an arbitrary "virtual sphere" of radius $r$. Note also that this definition is compatible with that given for the general static case above. (In a different direction this expression is also compatible with that for a time-dependent spherically symmetric situation as considered in [70].) As $r \rightarrow r_{H}$ this tends to the appropriate limit. For all $r$ this has the standard interpertation in terms of the tension in a massless rope supporting a small mass at radius $r$. A very standard computation now yields [31]

$$
\begin{aligned}
\rho= & \frac{m^{\prime}(r)}{4 \pi r^{2}} ; \quad p_{r}=-\frac{m^{\prime}(r)}{4 \pi r^{2}}-\frac{\Phi^{\prime}(r)}{4 \pi r}\left[1-\frac{2 m(r)}{r}\right] ; \\
p_{t}= & \frac{1}{8 \pi}\left\{-\frac{m^{\prime \prime}(r)}{r}-\Phi^{\prime}(r) \frac{1+m(r) / r-3 m^{\prime}(r)}{r}\right. \\
& \left.-\left[\Phi^{\prime \prime}(r)+\left(\Phi^{\prime}\right)^{2}\right]\left[1-\frac{2 m(r)}{r}\right]\right\} .
\end{aligned}
$$

By explicit integration one obtains (for any $r$ )

$$
\int_{0}^{r} e^{-\Phi(r)}\left\{\rho+p_{r}+2 p_{t}\right\} r^{2} \mathrm{~d} r=r^{2} \kappa(r) .
$$

That is, in spherical symmetry the partial Tolman mass of a star/planet out to radius $r$ has the very explicit form

$$
\begin{aligned}
& m_{T}(r)=r^{2} \kappa(r) \\
& \quad=e^{-\Phi(r)}\left\{m(r)-r m^{\prime}(r)-\Phi^{\prime}(r) r^{2}\left[1-\frac{2 m(r)}{r}\right]\right\} .
\end{aligned}
$$

Using

$$
\kappa(r)=e^{-\Phi(r)}\left\{\frac{m(r)}{r^{2}}+4 \pi r p_{r}\right\},
$$

it may be advantageous to rephrase this as

$$
m_{T}(r)=e^{-\Phi(r)}\left\{m(r)+4 \pi r^{3} p_{r}\right\} .
$$


The entropy inequalities still carry through in essentially the same way: For any virtual sphere of radius $r$ we have

$$
S(r) \leq \frac{m_{T}(r)}{T_{\infty}}=\frac{\kappa(r) r^{2}}{T_{\infty}}
$$

By considering FIDOs at radius $r$, the Unruh effect forces

$$
T_{\infty} \geq \frac{\kappa(r)}{2 \pi}
$$

so that

$$
S(r) \leq 2 \pi r^{2}
$$

with this inequality now holding for virtual spheres at arbitrary radii $r$. The inequality is again sub-optimal, (based on the holographic bound [45] we would have expected $S(r) \leq \pi r^{2}$ ), but on the other hand the inequality is extremely robust and easy to derive.

\subsubsection{Black hole region}

Now consider the situation where the region $\Omega$ contains a black hole region $\mathcal{B}$ with horizon $\partial \mathcal{B}$. It makes sense to now define

$$
m_{T}(\Omega)=m_{T}(\mathcal{B})+\int_{\Omega-\mathcal{B}} \sqrt{-g_{4}}\{\rho+3 p\} \mathrm{d}^{3} x
$$

where $m_{T}(\mathcal{B})$ is the so far undefined Tolman mass to be attributed to the black hole region $\mathcal{B}$. Appeal to the flux integral theorem, noting that $\partial(\Omega-$ $\mathcal{B})=\partial \Omega-\partial \mathcal{B}$, and using the zeroth law of black hole mechanics to assert that $\kappa(\partial \mathcal{B})$ is constant on the horizon $\partial \mathcal{B}[43]$, to write

$$
m_{T}(\Omega)=m_{T}(\mathcal{B})+\frac{1}{4 \pi} \int_{\partial \Omega} \vec{\kappa} \cdot \hat{n} \mathrm{~d} \mathscr{A}-\frac{\kappa(\partial \mathcal{B}) \mathscr{A}(\partial \mathcal{B})}{4 \pi} .
$$

If we now demand that $m_{T}(\Omega) \rightarrow m_{A D M}$ at spatial infinity then we uniquely have

$$
m_{T}(\mathcal{B})=\frac{\kappa(\partial \mathcal{B}) \mathscr{A}(\partial \mathcal{B})}{4 \pi}
$$


and

$$
m_{T}(\Omega)=\frac{1}{4 \pi} \int_{\partial \Omega} \vec{\kappa} \cdot \hat{n} \mathrm{~d} \mathscr{A}
$$

That is, with a suitable definition of $m_{T}(\mathcal{B})$ we can extend our flux formula for $m_{T}(\Omega)$ to situations where $\Omega$ contains a black hole region $\mathcal{B}$.

Finally, consider the total entropy

$$
S(\Omega)=S(\mathcal{B})+\int_{\Omega-\mathcal{B}} \sqrt{g_{3}} s \mathrm{~d}^{3} x,
$$

which we have divided into a geometrical entropy associated with the black hole region and a thermodynamic entropy associated with the surrounding matter. Again assuming internal equilibrium in the $\Omega-\mathcal{B}$ region, with non-negative pressure $p \geq 0$ and non-negative chemical potential $\mu \geq 0$, we obtain the bound

$$
S(\Omega) \leq S(\mathcal{B})+\frac{m_{T}(\Omega)-m_{T}(\mathcal{B})}{T_{\infty}} .
$$

But then our flux theorem gives

$$
S(\Omega) \leq S(\mathcal{B})+\frac{\int_{\partial \Omega} \vec{k} \cdot \hat{n} \mathrm{~d} \mathscr{A}-\kappa(\partial \mathcal{B}) \mathscr{A}(\partial \mathcal{B})}{4 \pi T_{\infty}}
$$

implying

$$
S(\Omega) \leq S(\mathcal{B})+\frac{\bar{\kappa}(\partial \Omega) \mathscr{A}(\partial \Omega)-\kappa(\partial \mathcal{B}) \mathscr{A}(\partial \mathcal{B})}{4 \pi T_{\infty}}
$$

But the very fact that we now know $\Omega$ contains a black hole region $\mathcal{B}$ implies that we can see down to the horizon $\partial \mathcal{B}$. Appealing to the Unruh argument, then at least in normal situations where the surface gravity increases as one moves inwards, $T_{\infty} \geq \frac{1}{2 \pi} \kappa(\partial \mathcal{B})$. Therefore

$$
S(\Omega) \leq \frac{\bar{\kappa}(\partial \Omega)}{\kappa(\partial \mathcal{B})} \frac{\mathscr{A}(\partial \Omega)}{2}+S(\mathcal{B})-\frac{\mathscr{A}(\partial \mathcal{B})}{2} .
$$

But then appealing to the ordinary Bekenstein result $S(\mathcal{B})=\frac{1}{4} \mathscr{A}(\partial \mathcal{B})$, but without any need to invoke the generalized second law or holographic bound, we have

$$
S(\Omega) \leq \frac{\bar{\kappa}(\partial \Omega)}{\kappa(\partial \mathcal{B})} \frac{\mathscr{A}(\partial \Omega)}{2}
$$


This is now a considerably tighter bound on the total entropy inside the region $\Omega$, using both information from the surface $\partial \Omega$, plus some information about the black hole region $\mathcal{B}$.

\subsection{Entropy bounds for uncollapsed rotating bodies}

Consider a rotating blob of matter that has not collapsed to a black hole. Can we nevertheless place a robust bound on its entropy using fundamental physics, without resorting to black hole physics (generalized second law [46], holographic bound [45], horizon entanglement entropy [51]) or the Bekenstein bound [47]?

In the case of non-rotating static configurations this was recently answered in the affirmative in section 3.1 (and in references [71, 72]) when we derived a bound based only on classical general relativity, basic thermodynamics, and the Unruh effect, to the effect that (entropy) $\leq \frac{1}{2}$ (area). The $\frac{1}{2}$ is not a typo - ultimately one has $\frac{1}{2}$ (rather than the $\frac{1}{4}$ one might naively expect based on black hole physics) simply because the matter has not collapsed to a black hole [71, 72]. We shall now extend this result to rotating blobs of uncollapsed matter - the derivation is slightly trickier than one might at first suppose, and the logic flow has to be somewhat reordered, but ultimately the basic result is the same: (entropy) $\leq \frac{1}{2}$ (area).

To start the discussion we appeal to the ordinary second law to note that the entropy of the rotating blob is less than what it will be once the blob has settled down to complete mechanical and thermodynamic equilibrium. So we might as well restrict attention to equilibrium configurations. But equilibrium configurations in general relativity have three absolutely crucial properties [73]. They are:

- Stationarity.

- Azimuthal symmetry. 
- Rigid rotation.

Stationarity basically means time-independence, a basic requirement of equilibrium configurations. Turning to the remaining two conditions: Physically, if the blob is not azimuthally symmetric, but is rotating, then it will emit gravitational radiation, thereby losing energy, so it cannot be in equilibrium. Thus equilibrium in general relativity implies the standard result that there are two Killing vectors, one timelike and one spacelike. (See for example [74, 75, 76].) Finally, if the blob is a solid, then "rigid rotation" is automatic. For a fluid the physics argument goes as follows: If the fluid blob is not rigidly rotating then velocity gradients imply shear, and shear implies friction, so the blob is losing energy, and cannot be in equilibrium. Thus in general relativity an equilibrium body cannot support any shear, and this will be our definition of rigid rotation. (See for example $[74,75,76])$. Since these are quite standard results we will simply use them and not further belabour the point.

The goal now is, within this particular framework, to derive robust bounds on the entropy content of the rotating blob. We shall first derive a purely classical upper bound on the entropy, using only classical general relativity and basic thermodynamics. This purely classical bound, because the terms being neglected are comparable to the terms being retained, is still relatively strong.

We then make our only appeal to quantum physics, using the Unruh effect to argue for a lower bound on the temperature based on the 4acceleration of a suitable defined class of fiducial observers [FIDOs]. Combining these results we argue that for uncollapsed matter there is a bound (entropy) $\leq \frac{1}{2}$ (area). Because of the relative weakness of the bound on the temperature, this entropy bound is also relatively weak unless the object of interest is extremely compact. Such bounds are of particular interest in view of recent speculations regarding monsters [54, 55, 56]/ gravastars [57, 58, 59, 60], black stars [61, 62, 63, 64], or quasi-black holes [65, 66]. 


\subsubsection{Metric}

In view of stationarity and azimuthal symmetry we can choose coordinates such that the metric takes the form

$$
\mathrm{d} s^{2}=g_{t t} \mathrm{~d} t^{2}+2 g_{t \phi} \mathrm{d} t \mathrm{~d} \phi+g_{\phi \phi} \mathrm{d} \phi^{2}+g_{r r} \mathrm{~d} r^{2}+g_{\theta \theta} \mathrm{d} \theta^{2},
$$

which is better written as

$$
\mathrm{d} s^{2}=-N^{2} \mathrm{~d} t^{2}+g_{\phi \phi}(\mathrm{d} \phi-\varpi \mathrm{d} t)^{2}+g_{r r} \mathrm{~d} r^{2}+g_{\theta \theta} \mathrm{d} \theta^{2} .
$$

Note that the metric components are independent of $t$ and $\phi$. The labels $r$ and $\theta$ for the remaining two coordinates are completely conventional and these coordinates can be shuffled around at will (as long as one does so in a $t$ and $\phi$ independent manner). Note that we use $-\varpi$ to denote the ratio of metric components $g_{t \phi} / g_{\phi \phi}$; the symbol $\omega$ will be reserved for the vorticity of a certain timelike congruence we shall subsequently encounter. This ADM decomposition for the stationary axially symmetric spacetime implies

$$
g_{a b}=\left[\begin{array}{cccc}
g_{t t} & g_{t \phi} & 0 & 0 \\
g_{t \phi} & g_{\phi \phi} & 0 & 0 \\
0 & 0 & g_{r r} & 0 \\
0 & 0 & 0 & g_{\theta \theta}
\end{array}\right]=\left[\begin{array}{cccc}
-\left[N^{2}-g_{\phi \phi} \varpi^{2}\right] & -g_{\phi \phi} \varpi & 0 & 0 \\
-g_{\phi \phi} \varpi & g_{\phi \phi} & 0 & 0 \\
0 & 0 & g_{r r} & 0 \\
0 & 0 & 0 & g_{\theta \theta}
\end{array}\right],
$$

and

$$
g^{a b}=\left[\begin{array}{cccc}
g^{t t} & g^{t \phi} & 0 & 0 \\
g^{t \phi} & g^{\phi \phi} & 0 & 0 \\
0 & 0 & g^{r r} & 0 \\
0 & 0 & 0 & g^{\theta \theta}
\end{array}\right]=\left[\begin{array}{cccc}
-1 / N^{2} & -\varpi / N^{2} & 0 & 0 \\
-\varpi / N^{2} & 1 / g_{\phi \phi}-\varpi^{2} / N^{2} & 0 & 0 \\
0 & 0 & 1 / g_{r r} & 0 \\
0 & 0 & 0 & 1 / g_{\theta \theta}
\end{array}\right] .
$$

Note also that on the rotation axis we have $g_{\phi \phi} \rightarrow 0$. 


\subsubsection{Matter}

The two natural Killing vectors are the timelike Killing vector

$$
\left(k_{T}\right)^{a}=\left(\partial_{t}\right)^{a}=(1,0,0,0),
$$

and the azimuthal Killing vector

$$
\left(k_{\Phi}\right)^{a}=\left(\partial_{\phi}\right)^{a}=(0,1,0,0) .
$$

Assuming the matter is a perfect fluid, with the stress-energy tensor taking the form

$$
T^{a b}=(\rho+p) u^{a} u^{b}+p g^{a b},
$$

with $u^{a}$ being the fluid four-velocity. Then internal equilibrium within the fluid ball demands [74, 75, 76]

$$
u^{a} \propto\left(k_{T}\right)^{a}+\Omega\left(k_{\Phi}\right)^{a},
$$

where $\Omega$ is constant throughout the fluid. That is, there is a comoving Killing vector

$$
\left(k_{C}\right)^{a}=\left(k_{T}\right)^{a}+\Omega\left(k_{\Phi}\right)^{a}=\left(\partial_{t}\right)^{a}+\Omega\left(\partial_{\phi}\right)^{a}=(1, \Omega, 0,0),
$$

such that

$$
u_{C}^{a}=\frac{\left(k_{C}\right)^{a}}{\left\|k_{C}\right\|} .
$$

It is trivial to see that the shear of this comoving 4-velocity $u_{C}$ is zero. The converse is slightly tedious but completely standard [74, 75, 76]. A further nontrivial and potentially useful observation is that $\Omega>\varpi$ is guaranteed throughout the interior of the blob via the so-called " $r$-mode instability" - if at any point internal to the fluid ball we have $\varpi>\Omega$ then the fluid blob cannot be in internal equilibrium [77, 78, 79]. Finally, observe that the comoving 4-velocity is spacelike far away from the axis of rotation - this is perfectly standard, and in particular implies a physical bound on the spatial size of the rotating blob - in the sense that the surface of the blob must have 3-velocity slower than the speed of light. 


\subsubsection{Thermodynamic equilibrium}

In a static spacetime the standard Tolman-Ehrenfest and Tolman-Klein [67, 68, 80] equilibrium conditions (see also [81, 42]) for the locally measured temperature and chemical potential are:

$$
T\left\|k_{T}\right\|=T_{\infty} ; \quad \mu\left\|k_{T}\right\|=\mu_{\infty} .
$$

Here $k_{T}$ is the timelike Killing vector, and in appropriate coordinates

$$
\left(k_{T}\right)^{a}=\left(\partial_{t}\right)^{a}=(1,0,0,0),
$$

so that we can rephrase things as

$$
T \sqrt{-g_{t t}}=T_{\infty} ; \quad \mu \sqrt{-g_{t t}}=\mu_{\infty} .
$$

(There is a minor technical assumption: we normalize $g_{t t} \rightarrow-1$ at spatial infinity.) Once we add rotation life gets a little more complicated. The internal equilibrium conditions are now given in terms of the comoving Killing vector

$$
T|| k_{C}\left\|=T_{\infty} ; \quad \mu\right\| k_{C} \|=\mu_{\infty} .
$$

In appropriate coordinates we can rephrase things as

$$
T \sqrt{-\left(g_{t t}+2 \Omega g_{t \phi}+\Omega^{2} g_{\phi \phi}\right)}=T_{\infty} ; \quad \mu \sqrt{-\left(g_{t t}+2 \Omega g_{t \phi}+\Omega^{2} g_{\phi \phi}\right)}=\mu_{\infty} .
$$

Note that $T_{\infty}$ and $\mu_{\infty}$ are now defined by first going onto the rotation axis and then moving to spatial infinity. (There is a minor technical assumption that $T_{\infty}$ at north and south polar infinities are the same, and similarly for $\mu_{\infty}$.) We can also write this as

$$
T \sqrt{N^{2}-g_{\phi \phi}(\Omega-\varpi)^{2}}=T_{\infty} ; \quad \mu \sqrt{N^{2}-g_{\phi \phi}(\Omega-\varpi)^{2}}=\mu_{\infty} .
$$

It is useful to note

$$
\left\|k_{T}\right\|=\sqrt{N^{2}-g_{\phi \phi} \varpi^{2}} ; \quad\left\|k_{\Phi}\right\|=\sqrt{g_{\phi \phi}} ; \quad\left\|k_{C}\right\|=\sqrt{N^{2}-g_{\phi \phi}(\Omega-\varpi)^{2}} .
$$

In particular

$$
\left\|k_{T}\right\| \leq N ; \quad\left\|k_{C}\right\| \leq N
$$




\subsubsection{Entropy current}

The entropy current (which is conserved because we are in equilibrium) is given in terms of the locally measured entropy density $s$ by

$$
S^{a}=s u_{C}^{a}=s\left\{\frac{\left[k_{T}\right]^{a}+\Omega\left[k_{\Phi}\right]^{a}}{\left\|k_{T}+\Omega k_{\Phi}\right\|}\right\} .
$$

This means the total entropy is

$$
S=\int s u_{C}^{t} \sqrt{-g_{4}} \mathrm{~d}^{3} x=\int s \frac{1}{\left\|k_{C}\right\|} \sqrt{-g_{4}} \mathrm{~d}^{3} x=\int s \frac{T}{T_{\infty}} \sqrt{-g_{4}} \mathrm{~d}^{3} x
$$

where we have used the Tolman equilibrium condition. Now apply the Euler relation

$$
s=\frac{\rho+p-\mu n}{T}
$$

to obtain

$$
S=\int \frac{\rho+p-\mu n}{T_{\infty}} \sqrt{-g_{4}} \mathrm{~d}^{3} x=\frac{1}{T_{\infty}} \int\{\rho+p-\mu n\} \sqrt{-g_{4}} \mathrm{~d}^{3} x .
$$

Similarly, the number density current is (in terms of comoving number density $n$ )

$$
j^{a}=n u_{C}^{a}=n\left\{\frac{\left[k_{T}\right]^{a}+\Omega\left[k_{\Phi}\right]^{a}}{\left\|k_{T}+\Omega k_{\Phi}\right\|}\right\} .
$$

This means the total number of particles is

$$
\mathscr{N}=\int n u^{t} \sqrt{-g_{4}} \mathrm{~d}^{3} x=\int n \frac{1}{\left\|k_{C}\right\|} \sqrt{-g_{4}} \mathrm{~d}^{3} x=\int n \frac{\mu}{\mu_{\infty}} \sqrt{-g_{4}} \mathrm{~d}^{3} x
$$

where we have used the Tolman-Klein equilibrium condition. That is

$$
\mathscr{N}=\frac{1}{\mu_{\infty}} \int n \mu \sqrt{-g_{4}} \mathrm{~d}^{3} x
$$

whence

$$
S=\frac{1}{T_{\infty}} \int\{\rho+p\} \sqrt{-g_{4}} \mathrm{~d}^{3} x-\frac{\mu_{\infty} \mathscr{N}}{T_{\infty}} .
$$


This is the same fundamental equation as we had for the non-rotating case [71, 72] — but the logic flow we used to get to it has been rather different. Assuming a non-negative chemical potential we still have

$$
S \leq \frac{1}{T_{\infty}} \int\{\rho+p\} \sqrt{-g_{4}} \mathrm{~d}^{3} x .
$$

As long as pressure is positive

$$
S \leq \frac{1}{T_{\infty}} \int\{\rho+3 p\} \sqrt{-g_{4}} \mathrm{~d}^{3} x .
$$

This is the same fundamental inequality as we had for the non-rotating case [71, 72] - but the interpretation will now be rather different. In the static case the quantity

$$
Q_{S} \equiv \int\{\rho+3 p\} \sqrt{-g_{4}} \mathrm{~d}^{3} x
$$

is equal to the so-called Tolman mass [81, 42]. In a rotating system this is no longer true. $Q_{S}$ is closely related to the Tolman mass but no longer equal to it. This is not a problem for us, as it is this quantity $Q_{S}$ that we shall now bound, and so use to produce a bound on the entropy. At this stage of the calculation we must be content with

$$
S \leq \frac{Q_{S}}{T_{\infty}}
$$

\subsubsection{Classical entropy bound}

To bound the quantity $Q_{S}$ it is useful to consider the two natural congruences on the spacetime.

- For the comoving congruence $u_{C}$ we have $u_{C} \propto k_{T}+\Omega k_{\Phi}$, so that we are dealing with a Killing congruence.

- The second natural congruence to consider is the congruence defined by the FIDOs, sometimes called ZAMOs (zero angular momentum observers). See for example [25]. This FIDO/ZAMO congruence is specified by $u_{F}=-(\mathrm{d} t)^{\#} /\|\mathrm{d} t\|$, or more explicitly $\left(u_{F}\right)^{a}=$ $-\nabla^{a} t /\|\nabla t\|$. 
Note that in stationary axial symmetry we have

$$
\left[u_{C}\right]^{a}=\frac{(1, \Omega, 0,0)}{\left\|k_{C}\right\|}
$$

while

$$
\left[u_{F}\right]^{a}=\frac{(1, \varpi, 0,0)}{N}
$$

and so in particular

$$
\nabla \cdot u_{F}=0
$$

Since $u_{C}$ and $u_{F}$ are both timelike we know

$$
\left(u_{C} \cdot u_{F}\right)^{2} \geq 1
$$

We now have

$$
\begin{aligned}
Q_{S} & =\int\{\rho+3 p\} \sqrt{-g_{4}} \mathrm{~d}^{3} x \\
& \leq \int\left\{2 \rho\left(u_{C} \cdot u_{F}\right)^{2}+(-\rho+3 p)\right\} \sqrt{-g_{4}} \mathrm{~d}^{3} x .
\end{aligned}
$$

Now using the conditions of internal equilibrium for a perfect fluid we have in particular,

$$
\begin{aligned}
T_{a b} u_{F}^{a} u_{F}^{b} & =\rho\left(u_{C} \cdot u_{F}\right)^{2}+p u_{F}\left(g+u_{C} u_{C}\right) u_{F} \\
& =\rho\left(u_{C} \cdot u_{F}\right)^{2}+p\left(1+\left(u_{C} \cdot u_{F}\right)^{2}\right) \\
& \geq \rho\left(u_{C} \cdot u_{F}\right)^{2} .
\end{aligned}
$$

Then we can write

$$
\begin{aligned}
Q_{S} & \leq \int\left\{2 T_{a b} u_{F}^{a} u_{F}^{b}+T\right\} \sqrt{-g_{4}} \mathrm{~d}^{3} x \\
& =2 \int\left\{T_{a b}-\frac{1}{2} T g_{a b}\right\} u_{F}^{a} u_{F}^{b} \sqrt{-g_{4}} \mathrm{~d}^{3} x \\
& =\frac{1}{4 \pi} \int\left\{R_{a b}\right\} u_{F}^{a} u_{F}^{b} \sqrt{-g_{4}} \mathrm{~d}^{3} x,
\end{aligned}
$$

where in the last step we have used the Einstein equations. Now by construction the congruence $u_{F}$ is irrotational (vorticity free, $\omega=0$ ), and in 
addition we have seen that it is divergence free, $\theta=0$. The congruence $u_{F}$ is however not a geodesic congruence, and we let $a_{F}$ be the 4-acceleration of $u_{F}$. It is now a standard result that the (non-geodesic) timelike Raychaudhuri equation (see chapter 4 ) implies

$$
R_{a b} u_{F}^{a} u_{F}^{b}=-\sigma^{2}+\nabla \cdot a_{F},
$$

where $\sigma$ is the shear of $u_{F}$. This implies

$$
Q_{S} \leq \frac{1}{4 \pi} \int\left\{-\sigma^{2}+\nabla \cdot a_{F}\right\} \sqrt{-g_{4}} \mathrm{~d}^{3} x .
$$

But we always have $\sigma^{2} \geq 0$, so we see

$$
\begin{aligned}
Q_{S} & \leq \frac{1}{4 \pi} \int\left\{\nabla \cdot a_{F}\right\} \sqrt{-g_{4}} \mathrm{~d}^{3} x \\
& =\frac{1}{4 \pi} \int\left\{\partial_{i}\left(\sqrt{-g_{4}} a_{F}^{i}\right)\right\} \mathrm{d}^{3} x \\
& =\frac{1}{4 \pi} \int\left\{\partial_{i}\left(\sqrt{g_{3}} N a_{F}^{i}\right)\right\} \mathrm{d}^{3} x \\
& =\frac{1}{4 \pi} \int\left\{N a_{F}^{i}\right\} \hat{n}_{i} \sqrt{g_{2}} \mathrm{~d}^{2} x .
\end{aligned}
$$

The index $i$ runs over $r, \theta, \phi$, since any $t$ dependence is automatically eliminated by stationarity. Now define a 3-dimensional vector

$$
\vec{\kappa}=N \vec{a}_{F}
$$

in terms of which we have

$$
Q_{S} \leq \frac{1}{4 \pi} \int\{\vec{\kappa} \cdot \hat{n}\} \sqrt{g_{2}} \mathrm{~d}^{2} x .
$$

In terms of the area, $\mathrm{d} \mathscr{A}=\sqrt{g_{2}} \mathrm{~d}^{2} x$, this implies

$$
Q_{S} \leq \frac{1}{4 \pi} \int\|\vec{\kappa}\| \mathrm{d} \mathscr{A}
$$

Note that $\vec{\kappa}$ is a natural generalization of the usual surface gravity [71, 72], which now extends into the bulk of any general stationary axisymmetric 
spacetime. (And so in particular $\vec{\kappa}$ makes sense for the 2-surface of any arbitrary 3-volume, regardless of whether or not that 2-surface is null.) It is interesting to note that there are a large number of situations for which similar "bulk" extensions of the usual notion of surface gravity are important [15, 82, 83].

Summarizing: Up to this stage of the argument, purely on classical grounds, (classical general relativity plus basic thermodynamics), we see that the entropy of equilibrium uncollapsed matter confined to a region of surface area $\mathscr{A}$ is bounded by

$$
S \leq \frac{Q_{S}}{T_{\infty}} \leq \frac{1}{4 \pi T_{\infty}} \int\|\vec{\kappa}\| \mathrm{d} \mathscr{A} \leq \frac{\|\vec{\kappa}\|_{\max (\text { surface })} \mathscr{A}}{4 \pi T_{\infty}}
$$

No appeal to quantum physics has yet been made. Note that the terms being neglected in deriving this bound, pressures and chemical potentials, are typically smaller than or the same order as the terms being retained. This purely classical bound is typically a reasonably tight bound on the entropy.

\subsubsection{Unruh temperature}

We now invoke the only bit of quantum physics that enters our argument: The Unruh effect [44]. This effect has now been studied for some 35 years and is closely related to the Hawking radiation effect [84, 85]. Like Hawking radiation, despite some 35 years of intense theoretical effort there has as yet been no fully convincing experimental proof of the reality of this effect - though the situation may now be changing [86, 87, 88, 89]. Nevertheless, the Unruh effect is based on such basic and fundamental aspects of special relativistic quantum field theory that it is extremely difficult to see how to avoid this effect without at the same time undermining many highly successful aspects of quantum field theory. Accordingly, while the Unruh effect may not have the direct experimental support of the various 
ingredients that went into the classical bound derived above, the existence of a quantum-induced Unruh temperature is felt (by almost everyone in the community) to be an entirely uncontroversial and plausible assumption.

Explicitly introducing the Boltzmann constant and Planck constant, for a FIDO with 4-velocity $u_{F}$ and 4-acceleration $a_{F}$ the locally measured Unruh temperature is

$$
k_{B} T_{U, F}=\frac{\hbar\left\|a_{F}\right\|}{2 \pi} .
$$

To convert this to a temperature as seen by a comoving observer $u_{C}$ the standard technique is to define a temperature 4-vector, $T^{a}=T u_{F}^{a}$, and perform a Lorentz transformation [42]. The relevant quantity is boosted by the gamma factor

$$
\gamma=\left|u_{F} \cdot u_{C}\right|=\frac{\left|\nabla t \cdot\left(k_{T}+\Omega k_{\Phi}\right)\right|}{\left\|\nabla t|| \mid k_{C}\right\|}=\frac{1}{(1 / N)\left\|k_{C}\right\|}=\frac{N}{\left\|k_{C}\right\|} \geq 1 .
$$

Thus the effective Unruh temperature associated with the FIDO $u_{F}$, as seen by a comoving observer $u_{C}$, is

$$
k_{B} T_{U, C}=\frac{\hbar\left\|a_{F}\right\| N}{2 \pi\left\|k_{C}\right\|} .
$$

Now, as seen from infinity along the axis of rotation we have seen that the relevant redshift factor (based on the Tolman-Ehrenfest relation [67, 68]) is

$$
T_{U, \infty}=T_{U, C}\left\|k_{C}\right\|
$$

So finally the Unruh temperature associated with the FIDO $u_{F}$, as seen from spatial infinity, is

$$
k_{B} T_{U, \infty}=\frac{\hbar\left\|a_{F}\right\| N}{2 \pi}=\frac{\hbar\|\vec{\kappa}\|}{2 \pi} .
$$

This implies that in any physical equilibrium system the Unruh effect can be used to argue for an ultimate and universal lower bound on the equilibrium temperature

$$
k_{B} T_{\infty} \geq \max _{\mathrm{FIDOs}}\left\{\frac{\hbar\|\vec{\kappa}\|}{2 \pi}\right\}
$$


We have explicitly included the $\hbar$ to make it absolutely clear that this appeal to the Unruh effect is the only part of the argument that in any way involves quantum physics. In more traditional theorists' units we can write this as

$$
T_{\infty} \geq \max _{\text {FIDOs }}\left\{\frac{\|\vec{\kappa}\|}{2 \pi}\right\} .
$$

Note that while formally this result is identical to the static spacetime result reported in section 3.1](Also in references [71, 72]), the technical issues underlying the argument are now considerably more subtle. Also note that this quantum-inspired semiclassical bound, while extremely general, is also often quantitatively weak: For objects such as stars or even planets, the actual temperature is often very many orders of magnitude higher than this quantum-inspired bound. Because of this, the resulting semiclassical entropy bound is sometimes rather weak - quantitatively weak but qualitatively robust. To get a tight quantitative bound on the temperature (and thus the entropy) we would need to consider some ultra-compact object (possibly a monster [54, 55, 56]/ gravastar [57, 58, 59, 60], black star [61, 62, 63, 64], or quasi-black hole [65, 66]) whose actual temperature was close to the Hawking temperature it would have if it were to collapse to a black hole.

\subsubsection{The semiclassical bound}

Having done all the preparatory work we finally note

$$
S \leq \frac{\|\vec{k}\|_{\max (\text { surface })} \mathscr{A}}{4 \pi T_{\infty}} ; \quad T_{\infty} \geq \frac{\|\vec{\kappa}\|_{\max (\text { FIDOs })}}{2 \pi} .
$$

Therefore

$$
S \leq\left\{\frac{\|\vec{\kappa}\|_{\max (\text { surface })}}{\|\vec{\kappa}\|_{\max (\text { FIDOs })}}\right\} \frac{\mathscr{A}}{2} .
$$

In particular, in the numerator we are maximizing only over those FIDOs that skim the surface of the object, while in the denominator we are maximizing over the larger class of all FIDOs in the bulk, therefore this brack- 
eted ratio is less than or equal to unity. Finally, as claimed,

$$
S \leq \frac{\mathscr{A}}{2}
$$

The particularly nice feature of this bound is how general it is and how weak the assumptions are that go into it. There are a number of places where the use of inequalities has been sub-optimal, and in situations where one knows more about the internal structure of the region enclosed by the surface of area $\mathscr{A}$ one might potentially be able to obtain tighter results. (See for example the discussion in reference [71].) However in general the $\frac{1}{2}$ seems to be an intrinsic feature for uncollapsed matter, ultimately arising from the use of the Euler relation, which in turn ultimately depends on temperature being intensive and entropy being extensive [71].

In counterpoint, note that for matter that has collapsed to a black hole one does not have the usual Euler relation. For example, for Schwarzschild black holes in general relativity one has $T \propto 1 / M$ and $S \propto M^{2}$. So temperature is no longer intensive and entropy is no longer extensive. The closest equivalent to the usual Euler relation for uncollapsed matter

$$
\rho=T s+\mu n-p,
$$

is (for collapsed matter) the Smarr mass formula for standard general relativity black holes [90, 43]

$$
M=2 T S+2 \Omega J+Q \Phi_{H}
$$

The key point here is the relative factor 2 between these two equations, which ultimately leads to (entropy) $\leq \frac{1}{2}$ (area) for uncollapsed matter.

\subsection{Discussion}

We have developed a number of entropy bounds that are very minimalist in the physics ingredients they require. The Einstein equations are used, 
mild conditions are placed on pressure and chemical potential, and the Unruh effect is invoked. Even with these rather minimal conditions, quite general and robust bounds can be extracted.

While we suspect that there might still be a number of ways in which (in specific situations) the bounds enunciated in this chapter can be improved, the overall message is (we think) clear: Useful entropy bounds can be derived from very basic physics without any recourse to the long sought for "full theory of quantum gravity". In the first part of the calculation we obtain a bound in a purely classical manner - using only classical general relativity and basic thermodynamics to place an upper bound on the entropy. A second kind of bound, summarized in equations (3.26) and (3.111), appealed to the Unruh effect (the only quantum physics involved in our argument) to place a lower bound on the temperature as seen at infinity. Combining these two bounds then yields our final result: (entropy) $\leq \frac{1}{2}$ (area) for uncollapsed matter. These are remarkably useful bounds based on an absolute minimum of physical assumptions.

Fundamentally the reason for this $\frac{1}{2} \leftrightarrow \frac{1}{4}$ mismatch is because we are looking at an uncollapsed distribution of matter, where the pressure does not present its most extreme behavior. Once the pressure becomes approximately equal to the density, the object is close to collapse and most of the mass in the system approaches the stiff matter limit [91]. In this high pressure regime equations (3.19) and (3.78) yield the more well-known entropy bound, $S \leq A / 4$. Although this argument is not yet rigorous, it presents an alternative way to further improve the entropy bounds developed in this chapter.

In addition, in this uncollapsed stage the temperature has its normal interpretation as an intensive variable, and the Euler relation takes the usual uncollapsed form $\rho=T s+\ldots$ which for a small element of matter integrates to $M=T S+\ldots$ In contrast, once the matter collapses to a black hole then (considering a Schwarzschild black hole for simplicity) $T=1 /(8 \pi M)$, so the temperature is no longer an intensive variable. Sim- 
ilarly the Bekenstein entropy $S=\frac{1}{4} \mathscr{A}=4 \pi M^{2}$ is no longer an extensive variable, and the Euler relation is modified to yield $M=2 T S+\ldots$ It is exactly this factor of 2 in the Euler relation for collapsed matter that prevents us from improving our entropy bound for uncollapsed matter to the tighter bound expected for collapsed matter. 



\section{4 The Raychaudhuri equation}

$\mathrm{T}$ The Raychaudhuri equation has become one of the standard workhorses of general relativity, particularly as applied to the singularity theorems. For textbook presentations see for instance [92, 29, 28, 93]. Nevertheless, there are still some interesting ways in which the general formalism can be extended. There are four extended versions of the Raychaudhuri equation that will be explored in this chapter:

- Single timelike unit vector field.

By collecting several terms in the usual formulation into a divergence, one can obtain a particularly useful version that finds many applications in the ADM formalism and other situations.

- Single spacelike unit vector field.

This situation is most typically ignored. A few hopefully clarifying comments will be made.

- Single non-normalized vector field.

This somewhat simplifies the Raychaudhuri equation, at the cost of no longer having nice positivity properties.

- Two non-normalized vector fields.

This allows one to probe off-diagonal components of the Ricci tensor. 
These four extensions of the Raychaudhuri equation will soon be seen to each be useful in their own way, and to yield different information. We shall provide numerous examples below.

\subsection{Single unit timelike vector field}

This is the standard case. Let $u^{a}$ be a field of unit timelike vectors (a congruence). This does not have to be the 4-velocity of a physical fluid (though it might be), it applies just as well to the 4-velocities of an imaginary collection of "fiducial observers" [FIDOs]. Then it is a purely geometrical result (see for example Hawking and Ellis [92], pp 82-84, or Wald [29], or Carroll [28], or Poisson [93], or even Wikipedia, (note that there are sometimes minor disagreements of notation - typically just a factor of 2 in odd places) that:

$$
\frac{\mathrm{d} \theta}{\mathrm{d} s}=-R_{a b} u^{a} u^{b}+\omega^{2}-\sigma^{2}-\frac{1}{3} \theta^{2}+\nabla_{a}\left(\frac{\mathrm{d} u^{a}}{\mathrm{~d} s}\right)
$$

This is the standard form of the Raychaudhuri equation. The spatial projection tensor is

$$
h_{a b}=g_{a b}+u_{a} u_{b}
$$

This projection tensor has signature $\{0,+1,+1,+1\}$. Various shear and expansion related quantities are

$$
\begin{gathered}
\theta_{a b}=h_{a c} \nabla^{(c} u^{d)} h_{d b} ; \\
\theta=g^{a b} \theta_{a b}=h^{a b} \theta_{a b}=\nabla_{a} u^{a} \\
\sigma_{a b}=\theta_{a b}-\frac{1}{3} h_{a b} \theta \\
\sigma^{2}=\sigma_{a b} \sigma^{a b} \geq 0 .
\end{gathered}
$$


Vorticity related quantities are

$$
\begin{gathered}
\omega_{a b}=h_{a c} \nabla^{[c} u^{d]} h_{d b} ; \\
\omega^{2}=\omega_{a b} \omega^{a b} \geq 0 .
\end{gathered}
$$

With these definitions we have the usual decomposition

$$
u_{a ; b}=\omega_{a b}+\sigma_{a b}+\frac{1}{3} \theta h_{a b}-\frac{\mathrm{d} u_{a}}{\mathrm{~d} s} u_{b} .
$$

See (for example) pages 82-84 of Hawking and Ellis [92]. Equation (4.1) is Wald's equation (9.2.11) [29], supplemented with the $\nabla_{a}\left(\frac{\mathrm{d} u^{a}}{\mathrm{~d} s}\right)$ term due to allowing a non-geodesic congruence; you can deduce the presence of this term from the second line in his (9.2.10) by not assuming geodesic motion.

Now consider the identity

$$
\frac{\mathrm{d} \theta}{\mathrm{d} s}=u \cdot \nabla \theta=\nabla \cdot(\theta u)-\theta \nabla \cdot u=\nabla \cdot(\theta u)-\theta^{2} .
$$

Using this identity we can also write the Raychaudhuri equation in the slightly unusual forms

$$
\nabla_{a}\left(\theta u^{a}-\frac{\mathrm{d} u^{a}}{\mathrm{~d} s}\right)=-R_{a b} u^{a} u^{b}+\omega^{2}-\sigma^{2}+\frac{2}{3} \theta^{2},
$$

or

$$
R_{a b} u^{a} u^{b}=\omega^{2}-\sigma^{2}+\frac{2}{3} \theta^{2}+\nabla_{a}\left(-\theta u^{a}+\frac{\mathrm{d} u^{a}}{\mathrm{~d} s}\right) .
$$

This extension of the usual Raychaudhuri equation is "close" to, but significantly more general than, a key technical result used by Padmanabhan and Patel in references [94, 48, 95].

\subsection{Applications}

We now consider several applications of the above formalism - these applications basically amount to strategically choosing an appropriate congruence. 


\subsubsection{Vorticity-free congruence}

Let $\Psi(x)$ be an arbitrary scalar field and define a set of fiducial observers [FIDOs] by

$$
u_{a} \propto \nabla_{a} \Psi .
$$

Then normalizing we have

$$
u_{a}=-\frac{\nabla_{a} \Psi}{\|\nabla \Psi\|}
$$

and furthermore

$$
\omega_{a b}=0 .
$$

The minus sign here is purely conventional, it guarantees that the $u^{a}$ is "future-pointing" in the direction of increasing $\Psi$. Conversely

$$
\omega_{a b}=0 \quad \Longrightarrow \quad u_{a} \propto \nabla_{a} \Psi .
$$

This is guaranteed by the Frobenius theorem.

Then in this vorticity-free situation the extended Raychaudhuri equation reduces to

$$
\frac{\mathrm{d} \theta}{\mathrm{d} s}=-R_{a b} u^{a} u^{b}-\sigma^{2}-\frac{1}{3} \theta^{2}+\nabla_{a}\left(\frac{\mathrm{d} u^{a}}{\mathrm{~d} s}\right),
$$

or equivalently

$$
R_{a b} u^{a} u^{b}=-\sigma^{2}+\frac{2}{3} \theta^{2}+\nabla_{a}\left(-\theta u^{a}+\frac{\mathrm{d} u^{a}}{\mathrm{~d} s}\right),
$$

or even

$$
R_{a b} u^{a} u^{b}=-\theta_{a b} \theta^{a b}+\theta^{2}+\nabla_{a}\left(-\theta u^{a}+\frac{\mathrm{d} u^{a}}{\mathrm{~d} s}\right) .
$$

But since $u^{a}$ is now hyper-surface orthogonal we can use the slices of constant $\Psi$ to define a spacelike foliation - the scalar $\Psi$ serves (at least locally) as a "cosmic time" function. Then in terms of the extrinsic curvature $K_{a b}$ of the constant $\Psi$ hyper-surfaces we have, (using Misner, Thorne, and Wheeler [96] sign conventions for the extrinsic curvature), the results:

$$
\theta_{a b}=-K_{a b} ; \quad \theta=-K ;
$$




$$
\begin{gathered}
\sigma_{a b}=-\left(K_{a b}-\frac{1}{3} K h_{a b}\right) \\
\sigma^{2}=\frac{1}{2}\left[K_{a b} K^{a b}-\frac{1}{3} K^{2}\right] .
\end{gathered}
$$

But then

$$
R_{a b} u^{a} u^{b}=-K_{a b} K^{a b}+K^{2}+\nabla_{a}\left(K u^{a}+\frac{\mathrm{d} u^{a}}{\mathrm{~d} s}\right) .
$$

This is effectively one of the key technical results used by Padmanabhan and Patel in [94, 48, 95], but now we see that this result is actually a special case of a considerably more general result, and can be viewed as a relatively straightforward extension and then specialization of the Raychaudhuri equation.

\subsubsection{ADM formalism}

By definition, in any stably causal spacetime there is a globally defined "cosmic time" function $t(x)$ such that $\mathrm{d} t$ is always timelike. Then on the one hand the constant- $t$ slices are always spacelike and can be used to set up an ADM decomposition of the metric, while on the other hand $u=-(\mathrm{d} t)^{\sharp} /\|\mathrm{d} t\|$ is a vorticity-free unit timelike congruence, so that the results of the previous subsection apply. (As usual, $\mathrm{d} t^{\sharp}$ denotes the vector obtained form the one-form $\mathrm{d} t$ by "raising the index", similarly $u^{b}$ will denote the one-form obtained from the vector $u$ by "lowering the index.)

Consequently the extended Raychaudhuri equation can now be cast in the form

$$
R_{\hat{t} \hat{t}}=-K_{a b} K^{a b}+K^{2}+\nabla_{a}\left(K u^{a}+\frac{\mathrm{d} u^{a}}{\mathrm{~d} s}\right) .
$$

This result complements and reinforces the information one obtains from the Gauss equations - see for example Misner, Thorne, and Wheeler [96] pp 505-520, or Rendall [97] pp 23-24. The Gauss equations (for a spacelike 
hypersurface) are

$$
{ }^{(4)} R_{a b c d}={ }^{(3)} R_{a b c d}+K_{a c} K_{b d}-K_{a d} K_{b c} .
$$

Contracting once

$$
{ }^{(4)} R_{a b}={ }^{(3)} R_{a b}-{ }^{(4)} R_{a c b d} u^{c} u^{d}+\operatorname{tr}(K) K_{a b}-\left(K^{2}\right)_{a b} .
$$

Contracting a second time

$$
{ }^{(4)} R={ }^{(3)} R-2^{(4)} R_{a b} u^{a} u^{b}+K^{2}-\operatorname{tr}\left(K^{2}\right) .
$$

But now, since ${ }^{(4)} R_{a b} u^{a} u^{b}$ has been given to us via the extended Raychaudhuri equation, we easily see that for a spacelike hypersurface

$$
{ }^{(4)} R={ }^{(3)} R+\operatorname{tr}\left(K^{2}\right)-K^{2}-2 \nabla_{a}\left(K u^{a}+\frac{\mathrm{d} u^{a}}{\mathrm{~d} s}\right) .
$$

Traditional derivations of this result are sometimes somewhat less transparent, and viewing it as an extension of the timelike Raychaudhuri equation is the cleanest derivation we have been able to develop. To see some of the deeper connections with the ADM formalism read (for example) $\S 21.6$ on pp 519-520 of Misner, Thorne, and Wheeler [96]; note especially eq (21.88). See also exercise (21.10) on p 519. Also note the discussion by Padmanabhan and Patel in references [94, 48, 95]. Also, we should warn the reader that Wald uses an opposite sign convention for the extrinsic curvature. See specifically Wald [29] equation (10.2.13) on page 256.

\subsubsection{Static spacetimes}

Let us now take the discussion in a rather different direction, and assume that the spacetime is static. That is, there exists a hypersurface-orthogonal Killing vector $k^{a}$ that is timelike at spatial infinity. Because it is hypersurface orthogonal then $k_{a} \propto \nabla_{a} \Psi$, and so $u^{a}=k^{a} /\|k\|$ is a set of FIDOs of 
the type considered in the previous section. But since $k^{a}$ is also a Killing vector we have $k_{(a ; b)}=0$ and so obtain the quite standard result that

$$
\begin{aligned}
u_{(a ; b)} & =\nabla_{(a}\{k /\|k\|\}_{b)}=\frac{k_{(a ; b)}}{\|k\|}-\frac{k_{(b} \nabla_{a)}\|k\|}{\|k\|^{2}} \\
& =-\frac{k_{(b} \nabla_{a)}\|k\|}{\|k\|^{2}}=-\frac{u_{(b} \nabla_{a)}\|k\|}{\|k\|} \\
& =-\frac{u_{(b}\|k\|_{, a)}}{\|k\|}=\frac{u_{(a}\|k\|_{, b)}}{\|k\|} .
\end{aligned}
$$

Hence

$$
\theta_{a b}=0 \quad \Longrightarrow \quad K_{a b}=0 \quad \Longrightarrow \quad K=0 .
$$

That is, in static spacetimes the extrinsic curvature of the time-slices is zero (in addition to the congruence being vorticity free). The Raychaudhuri equation then specializes to the particularly simple result

$$
R_{a b} u^{a} u^{b}=\nabla_{a}\left(\frac{\mathrm{d} u^{a}}{\mathrm{~d} s}\right)
$$

This is essentially the technical result previously used in the derivation of an entropy bound for static spacetimes in section 3.1 and in [71], though before it was derived from an old result due to Landau and Lifshitz [53]. (The original Landau-Lifshitz result is obtained via a straightforward but tedious series of index manipulations, with little geometrical insight.). In reference [72] the derivation presented here is used in a straightforward manner.

\subsubsection{Stationary spacetime - Killing congruence}

What can we now do for stationary, as opposed to static spacetimes? (This distinction is relevant to "rotating spacetimes", for example Kerr spacetimes versus Schwarzschild spacetimes. See for instance [98, 99, 100, 101].) The (asymptotically) timelike Killing vector $k=\partial_{t}$ [that is, $k^{a}=(1 ; 0,0,0)^{a}$ ] is no longer hypersurface orthogonal. Nevertheless we can still define the 
timelike Killing congruence

$$
u^{a}=\frac{k^{a}}{\|k\|}
$$

This timelike congruence corresponds to a class of FIDOs [not ZAMOs, not zero angular momentum observers] that sit at fixed spatial coordinate position [25] (Chapter 3). This timelike congruence, even though it is not hypersurface orthogonal, still satisfies equation (4.29). So even though there is no longer any interpretation of the shear in terms of an extrinsic curvature, we still have

$$
\theta_{a b}=0,
$$

whence both

$$
\sigma_{a b}=0 ; \quad \text { and } \quad \theta=0 .
$$

Therefore

$$
R_{a b} u^{a} u^{b}=\omega^{2}+\nabla_{a}\left(\frac{\mathrm{d} u^{a}}{\mathrm{~d} s}\right) .
$$

However, unless further assumptions are made, we cannot do much with the $\omega^{2}$ term. Generically we have

$$
\begin{aligned}
u_{[a ; b]} & =\nabla_{[a}\{k /\|k\|\}_{b]}=\frac{k_{[a ; b]}}{\|k\|}-\frac{k_{[b} \nabla_{a]}\|k\|}{\|k\|^{2}} \\
& =\frac{k_{[a ; b]}}{\|k\|}-\frac{k_{[b} \nabla_{a]}\|k\|}{\|k\|^{2}}=\frac{k_{[a ; b]}}{\|k\|}-\frac{u_{[b} \nabla_{a]}\|k\|}{\|k\|} \\
& =\frac{k_{[a ; b]}}{\|k\|}-\frac{u_{[b}\|k\|_{, a]}}{\|k\|} .
\end{aligned}
$$

This implies

$$
\omega^{a b}=h^{a c} h^{b d} \frac{k_{[c ; d]}}{\|k\|}
$$

whence

$$
R_{a b} u^{a} u^{b}=+\frac{h^{a c} h^{b d} k_{[a ; b]} k_{[c ; d]}}{\|k\|^{2}}+\nabla_{a}\left(\frac{\mathrm{d} u^{a}}{\mathrm{~d} s}\right) .
$$

Unfortunately this does not simplify any further, and without further assumptions for the timelike Killing congruence on a stationary spacetime 
we should just be satisfied by the inequality:

$$
R_{a b} u^{a} u^{b} \geq \nabla_{a}\left(\frac{\mathrm{d} u^{a}}{\mathrm{~d} s}\right)
$$

\subsubsection{Stationary axisymmetric spacetimes}

In a stationary axisymmetric spacetime consider the vorticity-free congruence of section 4.2.1 (not the Killing congruence of section 4.2.4). Because of the axisymmetry the congruence $u=-(\mathrm{d} t)^{\sharp} / \| \mathrm{d} t||$ must then be a linear combination of the two Killing vectors, $k_{t}=\partial_{t}$ and $k_{\phi}=\partial_{\phi}$, in which case $\theta=\nabla \cdot u=0$. In this case equation (4.18) reduces to

$$
R_{a b} u^{a} u^{b}=-\sigma^{2}+\nabla_{a}\left(\frac{\mathrm{d} u^{a}}{\mathrm{~d} s}\right)
$$

which implies, for the natural vorticity-free congruence on an stationary axisymmetric spacetime

$$
R_{a b} u^{a} u^{b} \leq \nabla_{a}\left(\frac{\mathrm{d} u^{a}}{\mathrm{~d} s}\right)
$$

It is this particular inequality that was used in Chapter 3 to place an entropy bound on rotating fluid blobs. (Note that the direction of the inequality has changed between equations (4.39) and (4.41), but that is merely due to the fact that we are using different timelike congruences.)

\subsection{Single unit spacelike vector field}

In counterpoint, we now let $u^{a}$ be a field of unit spacelike vectors. The projection tensor becomes

$$
h_{a b}=g_{a b}-u_{a} u_{b}
$$

In contrast to the timelike situation the projection tensor is now of indefinite signature $\{-1,+1,+1,0\}$. One can still formally define the quantities 
$\theta_{a b}, \theta, \sigma_{a b}$, and $\omega_{a b}$, but they no longer have the same physical interpretation in terms of shear and vorticity. Furthermore since the projection tensor has indefinite signature we now cannot guarantee either $\sigma^{2} \geq 0$ or $\omega^{2} \geq 0$. On the other hand, the Raychaudhuri equation is formally unaffected. That is, the fundamental equations (4.1), (4.11), and (4.12), continue to hold as they stand.

If we now consider a vorticity-free spacelike congruence, it will be hypersurface orthogonal to a timelike hypersurface. (That is, the normal to the hypersurface is spacelike, while the tangent space to the hypersurface can be chosen to have a basis of one timelike and two spacelike tangent vectors.)

In this situation we can without loss of generality set $u=(\mathrm{d} \Psi)^{\sharp} /\|\mathrm{d} \Psi\|$. Then $\omega_{a b} \rightarrow 0$, while in terms of the extrinsic curvature $\sigma_{a b} \rightarrow-K_{a b}$ as for vorticity free timelike congruencies. Thus equation 4.23 is formally unaffected and can now be cast in the form

$$
R_{\hat{n} \hat{n}}=-K_{a b} K^{a b}+K^{2}+\nabla_{a}\left(K u^{a}+\frac{\mathrm{d} u^{a}}{\mathrm{~d} s}\right) .
$$

On the other hand, because $u$ is now a spacelike normal to a timelike hypersurface there is a key sign flip in the Gauss equations, which now read

$$
{ }^{(4)} R_{a b c d}={ }^{(3)} R_{a b c d}-K_{a c} K_{b d}+K_{a d} K_{b c} .
$$

Contracting twice

$$
{ }^{(4)} R={ }^{(3)} R+2^{(4)} R_{a b} u^{a} u^{b}+\operatorname{tr}\left(K^{2}\right)-K^{2} .
$$

Therefore for a timelike hypersurface we have

$$
{ }^{(4)} R={ }^{(3)} R-\operatorname{tr}\left(K^{2}\right)+K^{2}+2 \nabla_{a}\left(K u^{a}+\frac{\mathrm{d} u^{a}}{\mathrm{~d} s}\right) .
$$

In summary, for spacelike congruences the Raychaudhuri equation itself is formally unaffected (though the projection tensor is slightly different and we can no longer rely on the non-negativity of $\sigma^{2}$ and $\omega^{2}$ ). How- 
ever applications of the Raychaudhuri equation, specifically anything involving the Gauss equations for embedded hypersurfaces, typically exhibit a limited number of sign flips.

\subsection{Single non-normalized vector field}

Now consider an non-normalized vector field $u^{a}$, either spacelike, timelike, or null. What if anything can we say about the quantity

$$
R_{a b} u^{a} u^{b}=? ? ?
$$

Following and modifying the discussion of Wald [29], see (E.2.28) on page 464:

$$
\begin{aligned}
R_{a b} u^{a} u^{b}= & R_{a c b}^{c} u^{a} u^{b} \\
= & -u^{a}\left[\nabla_{a} \nabla_{b}-\nabla_{b} \nabla_{a}\right] u^{b} \\
= & -\nabla_{a}\left(u^{a} \nabla_{b} u^{b}\right)+\left(\nabla_{a} u^{a}\right)\left(\nabla_{b} u^{b}\right) \\
& +\nabla_{b}\left(u^{a} \nabla_{a} u^{b}\right)-\left(\nabla_{b} u^{a}\right)\left(\nabla_{a} u^{b}\right) \\
= & \nabla_{a}\left(-u^{a} \nabla_{b} u^{b}+u^{b} \nabla_{b} u^{a}\right) \\
& +(\nabla \cdot u)^{2}-\left(\nabla_{b} u_{a}\right)\left(\nabla^{a} u^{b}\right) \\
= & \nabla \cdot\{(u \cdot \nabla) u-(\nabla \cdot u) u\} \\
& +(\nabla \cdot u)^{2}-\left(\nabla_{b} u_{a}\right)\left(\nabla^{a} u^{b}\right) .
\end{aligned}
$$

In obvious notation, using $\theta=\nabla \cdot u$, this can be cast as

$$
\begin{aligned}
R_{a b} u^{a} u^{b}= & \nabla \cdot\left\{\nabla_{u} u-\theta u\right\}+\theta^{2} \\
& -\nabla_{(a} u_{b)} \nabla^{(a} u^{b)}+\nabla_{[a} u_{b]} \nabla^{[a} u^{b]} .
\end{aligned}
$$

This result can be viewed as another generalization of the Raychaudhuri equation. The advantage of this particular formula is that we have not carried out any projections, and have not even committed ourselves to the 
nature of the congruence, be it spacelike, timelike, or null. One disadvantage is that because of the Lorentzian signature of spacetime we cannot (at least not without further assumption) guarantee

$$
\begin{aligned}
& \nabla_{(a} u_{b)} \nabla^{(a} u^{b)} \geq 0 \quad ? ? ? \\
& \nabla_{[a} u_{b]} \nabla^{[a} u^{b]} \geq 0 \quad ? ? ?
\end{aligned}
$$

Two specific applications come readily to mind:

- For any Killing vector $u^{a}=k^{a}$ we have $\nabla_{(a} u_{b)}=0$, and consequently $\theta=0$. Therefore for any Killing vector whatsoever

$$
R_{a b} k^{a} k^{b}=\nabla \cdot\left\{\nabla_{k} k\right\}+\nabla_{[a} k_{b]} \nabla^{[a} k^{b]} .
$$

- For any one arbitrary exact one-form $u=d \Psi$, even a locally exact one-form, we have $\nabla_{[a} u_{b]}=0$, while $\theta=\nabla^{2} \Psi$ and $\nabla_{(a} u_{b)} \nabla^{(a} u^{b)}=$ $\Psi_{; a ; b} \Psi^{; a ; b}$. Therefore for any locally exact one-form whatsoever

$$
\begin{aligned}
R^{a b}(\mathrm{~d} \Psi)_{a}(\mathrm{~d} \Psi)_{b}= & \nabla \cdot\left\{\nabla_{\mathrm{d} \Psi} \mathrm{d} \Psi-\left(\nabla^{2} \Psi\right) \mathrm{d} \Psi\right\} \\
& +\left(\nabla^{2} \Psi\right)^{2}-\Psi_{; a ; b} \Psi^{; a ; b} .
\end{aligned}
$$

In fact, $\Psi$ could simply be one of the spacetime coordinates (defined on some suitable local coordinate patch) in which case this version of the Raychaudhuri equation turns into a statement about the diagonal components of the Ricci tensor in a coordinate basis

$$
\begin{aligned}
R^{\Psi \Psi}= & \nabla \cdot\left\{\nabla_{\mathrm{d} \Psi} \mathrm{d} \Psi-\left(\nabla^{2} \Psi\right) \mathrm{d} \Psi\right\} \\
& +\left(\nabla^{2} \Psi\right)^{2}-\Psi_{; a ; b} \Psi^{; a ; b} .
\end{aligned}
$$

More boldly, if one chooses $\Psi$ to be a harmonic coordinate, $\left(\nabla^{2} \Psi=\right.$ 0 ), and this can always be done locally, then

$$
R^{\Psi \Psi}=\nabla \cdot\left\{\nabla_{\mathrm{d} \Psi} \mathrm{d} \Psi\right\}-\Psi_{; a ; b} \Psi^{; a ; b} .
$$

In summary, this extension of the Raychaudhuri equation has given us some useful computational formulae. 


\subsection{Two non-normalized vector fields}

We shall now ask if it is possible to extract any useful information by considering two different congruences simultaneously.

\subsubsection{Motivation}

To motivate this particular extension of the Raychaudhuri equation, recall that many decades ago Landau and Lifshitz had shown that in any stationary spacetime [53] ( $\$ 105$ equation (105.22), for a recent application of this result see section 3.1 and [71, 72]):

$$
R_{0}^{0}=\frac{1}{\sqrt{-g_{4}}} \partial_{i}\left(\sqrt{-g_{4}} g^{0 a} \Gamma_{a 0}^{i}\right) .
$$

(Here $a \in\{0,1,2,3\} ; i \in\{1,2,3\}$.) But because the metric is stationary ( $t$ independent) we can also write this as

$$
R_{0}^{0}=\frac{1}{\sqrt{-g_{4}}} \partial_{b}\left(\sqrt{-g_{4}} g^{0 a} \Gamma_{a 0}^{b}\right) .
$$

To begin converting this into a coordinate-free statement, note that

$$
R_{0}^{0}=R_{b}^{a}(\mathrm{~d} t)_{a}\left(\partial_{t}\right)^{b}=R_{b}^{a}(\mathrm{~d} t)_{a} k^{b} .
$$

Here we have had to use both the timelike Killing vector $k$, for which $k^{a}=$ $\left(\partial_{t}\right)^{a}=(1,0,0,0)^{a}$, and the one-form $\mathrm{d} t$, for which $(\mathrm{d} t)_{a}=(1,0,0,0)_{a}$. By direct computation

$$
\begin{aligned}
g^{0 a} \Gamma_{a 0}^{b} & =g^{c a} \Gamma_{a d}^{b}(\mathrm{~d} t)_{c} k^{d}=\Gamma_{c d}^{b}(\mathrm{~d} t)^{c} k^{d} \\
& =\Gamma_{c d}^{b} k^{c}(\mathrm{~d} t)^{d}=\left\{\partial_{d} k^{b}+\Gamma^{b}{ }_{c d} k^{c}\right\}(\mathrm{d} t)^{d} \\
& =\left(\nabla_{d} k^{b}\right)(\mathrm{d} t)^{d}=(\mathrm{d} t)^{d}\left(\nabla_{d} k^{b}\right) .
\end{aligned}
$$

But then

$$
\begin{aligned}
R_{0}{ }^{0} & =\frac{1}{\sqrt{-g_{4}}} \partial_{b}\left(\sqrt{-g_{4}} g^{0 a} \Gamma_{a 0}^{b}\right) \\
& =\frac{1}{\sqrt{-g_{4}}} \partial_{b}\left(\sqrt{-g_{4}}(\mathrm{~d} t)^{d}\left(\nabla_{d} k^{b}\right)\right) \\
& =\nabla_{b}\left\{(\mathrm{~d} t)^{d}\left(\nabla_{d} k^{b}\right)\right\} .
\end{aligned}
$$


So the Landau-Lifshitz result is equivalent to the statement that in any stationary spacetime

$$
R_{b}^{a}(\mathrm{~d} t)_{a} k^{b}=\nabla_{b}\left\{(\mathrm{~d} t)^{d}\left(\nabla_{d} k^{b}\right)\right\}=\nabla \cdot\left(\nabla_{\mathrm{d} t^{\sharp}} k\right) .
$$

So some linear combination of Ricci tensor components is given by a pure divergence. Note that two different vector fields are involved. This observation naturally leads to the question: Is it possible to come up with a variant of the Raychaudhuri equation that depends on two congruences $u^{a}$ and $v^{a}$ ? Something of the form

$$
R_{a b} u^{a} v^{b}=? ? ?
$$

We shall see how this is done below.

For now, let us mention that

$$
\begin{aligned}
\left(\nabla_{d} k^{b}\right)(\mathrm{d} t)^{d} & =\left(\nabla^{d} k^{b}\right)(\mathrm{d} t)_{d} \\
& =-\left(\nabla^{b} k^{d}\right)(\mathrm{d} t)_{d} \\
& =-\nabla^{b}\left\{k^{d}(\mathrm{~d} t)_{d}\right\}+k^{d} \nabla^{b}(\mathrm{~d} t)_{d} \\
& =-\nabla^{b}\{1\}+k^{d} \nabla^{b} \nabla_{d} t \\
& =k^{d} \nabla^{b} \nabla_{d} t \\
& =k^{d} \nabla_{d} \nabla^{b} t .
\end{aligned}
$$

That is,

$$
\nabla_{\mathrm{d} t^{\sharp}} k=\nabla_{k} \mathrm{~d} t^{\sharp} .
$$

So the Landau-Lifshitz result can also be written in the alternative form

$$
R_{b}^{a}(\mathrm{~d} t)_{a} k^{b}=\nabla_{b}\left\{k^{d} \nabla_{d} \nabla^{b} t\right\}=\nabla \cdot\left(\nabla_{k} \mathrm{~d} t^{\sharp}\right) .
$$

Finally note that

$$
\left(\nabla_{d} k^{b}\right)(\mathrm{d} t)^{d}(\mathrm{~d} t)_{b}=\left(\nabla_{d} k_{b}\right)(\mathrm{d} t)^{d}(\mathrm{~d} t)^{b}=0,
$$

so the vector $\nabla_{\mathrm{d} t} k$ is perpendicular to $\mathrm{d} t^{\sharp}$. 


\subsubsection{Construction}

Following and modifying the discussion of Wald [29], see equation (E.2.28) on page 464:

$$
\begin{aligned}
R_{a b} u^{a} v^{b} & =R_{a c b}^{c} u^{a} v^{b} \\
& =-u^{a}\left[\nabla_{a} \nabla_{b}-\nabla_{b} \nabla_{a}\right] v^{b} \\
& =-\nabla_{a}\left(u^{a} \nabla_{b} v^{b}\right)+\left(\nabla_{a} u^{a}\right)\left(\nabla_{b} v^{b}\right)+\nabla_{b}\left(u^{a} \nabla_{a} v^{b}\right)-\left(\nabla_{b} u^{a}\right)\left(\nabla_{a} v^{b}\right) \\
& =\nabla_{a}\left(-u^{a} \nabla_{b} v^{b}+u^{b} \nabla_{b} v^{a}\right)+(\nabla \cdot u)(\nabla \cdot v)-\left(\nabla_{b} u_{a}\right)\left(\nabla^{a} v^{b}\right) .
\end{aligned}
$$

With minor notational changes and given the symmetry of the Ricci tensor, this can also be written as

$$
\begin{aligned}
R_{a b} u^{a} v^{b}= & \nabla \cdot\{(u \cdot \nabla) v-(\nabla \cdot v) u\} \\
& +(\nabla \cdot u)(\nabla \cdot v)-\left(\nabla_{b} u_{a}\right)\left(\nabla^{a} v^{b}\right),
\end{aligned}
$$

and

$$
\begin{aligned}
R_{a b} u^{a} v^{b}= & \nabla \cdot\{(v \cdot \nabla) u-(\nabla \cdot u) v\} \\
& +(\nabla \cdot u)(\nabla \cdot v)-\left(\nabla_{b} u_{a}\right)\left(\nabla^{a} v^{b}\right) .
\end{aligned}
$$

Furthermore (in obvious notation) this can again be rewritten as

$$
\begin{aligned}
R_{a b} u^{a} v^{b}= & \nabla \cdot\left\{\nabla_{u} v-\theta_{v} u\right\}+\theta_{u} \theta_{v} \\
& -\nabla_{(a} u_{b)} \nabla^{(a} v^{b)}+\nabla_{[a} u_{b]} \nabla^{[a} v^{b]} .
\end{aligned}
$$

and

$$
\begin{aligned}
R_{a b} u^{a} v^{b}= & \nabla \cdot\left\{\nabla_{v} u-\theta_{u} v\right\}+\theta_{u} \theta_{v} \\
& -\nabla_{(a} u_{b)} \nabla^{(a} v^{b)}+\nabla_{[a} u_{b]} \nabla^{[a} v^{b]} .
\end{aligned}
$$

Note the similarities to the single-congruence case, and note particularly the presence of a divergence term generalizing the standard Raychaudhuri equation. To check the equivalence of these two formulae note

$$
\begin{aligned}
\left(\nabla_{u} v-\theta_{v} u\right)-\left(\nabla_{v} u-\theta_{u} v\right) & =\left[\nabla_{u} v+\theta_{u} v\right]-\left[\nabla_{v} u+\theta_{v} u\right] \\
& =\nabla \cdot[u \otimes v-v \otimes u] \\
& =\nabla \cdot[u \wedge v] .
\end{aligned}
$$


That is, the difference of these two currents is the divergence of a 2-form, which makes it automatically closed.

\subsubsection{Generalizing the Landau-Lifshitz result}

Let $u=k$ be any Killing vector, and let $v^{b}$ be an arbitrary (locally) exact one-form, so $v=(\mathrm{d} \Psi)^{\sharp}$ where $\Psi(x)$ is an arbitrary scalar. Then $\nabla_{(a} u_{b)}=$ $\nabla_{(a} k_{b)}=0$, and so $\theta_{u}=0$. Furthermore $\nabla_{[a} v_{b]}=\nabla_{[a} \nabla_{b]} \Psi=0$, so from equation (4.70) we have

$$
R_{a b} k^{a} \nabla^{b} \Psi=\nabla \cdot\left\{\nabla_{k} \mathrm{~d} \Psi^{\sharp}-\left(\nabla^{2} \Psi\right) k\right\},
$$

while from equation (4.71) we have

$$
R_{a b} k^{a} \nabla^{b} \Psi=\nabla \cdot\left\{\nabla_{\mathrm{d} \Psi \sharp} k\right\} .
$$

This nicely generalizes the Landau-Lifshitz result to any arbitrary Killing vector and any arbitrary (locally) exact one form $\mathrm{d} \Psi$, not just $\mathrm{d} t$. (That these two formulae are equivalent follows from the discussion in the previous section above.) Note the (standard) Landau-Lifshitz result corresponds to $k^{a} \rightarrow\left(\partial_{t}\right)^{a}$ and $\Psi \rightarrow t$.

Now choose a coordinate system adapted to the Killing vector $k$. Let $k=\partial_{\mathrm{K}}$ define a Killing coordinate $\mathrm{K}$, so that all geometrical objects are independent of the coordinate $\mathrm{K}$. Let $\Psi$ also be viewed as a coordinate, relabel it as $x^{a}$, possibly distinct from $\mathrm{K}$, and with no claim that $x^{a}$ necessarily corresponds to a Killing vector. Then

$$
R_{\mathrm{K}}{ }^{a}=\nabla \cdot\left\{\nabla_{\left(\mathrm{d} x^{a}\right)^{\sharp}} \partial_{\mathrm{K}}\right\} .
$$

Unwrapping the covariant derivatives we see

$$
R_{\mathrm{K}}^{a}=\frac{1}{\sqrt{-g_{4}}} \partial_{b}\left(\sqrt{-g_{4}} g^{a c} \Gamma_{c \mathrm{~K}}^{b}\right) .
$$

If we now let the index $i$ range over every coordinate except the Killing coordinate $\mathrm{K}$ then, because all geometrical objects are independent of the 
coordinate $\mathrm{K}$, we have

$$
R_{\mathrm{K}}^{a}=\frac{1}{\sqrt{-g_{4}}} \partial_{i}\left(\sqrt{-g_{4}} g^{a c} \Gamma_{c \mathrm{~K}}^{i}\right) .
$$

This equation, ultimately based on our two-congurence extension of the Raychaudhuri equation (4.70), is now very much in Landau-Lifshitz form, but it is definitely considerably more powerful than the original LandauLifshitz result.

\subsubsection{Landau-Lifshitz in axial symmetry}

Since in a stationary spacetime with axial symmetry we have a second azimuthal Killing vector $k^{a} \rightarrow\left(\partial_{\phi}\right)^{a}$, and could also consider $\Psi \rightarrow \phi$, then there are three additional Landau-Lifshitz like results:

$$
\begin{gathered}
R_{\phi}^{t}=R_{a b}\left(\partial_{\phi}\right)^{a} \nabla^{b} t=\nabla \cdot\left\{\nabla_{\mathrm{d} t^{\sharp}} \partial_{\phi}\right\} ; \\
R_{t}^{\phi}=R_{a b}\left(\partial_{t}\right)^{a} \nabla^{b} \phi=\nabla \cdot\left\{\nabla_{\mathrm{d} \phi^{\sharp}} \partial_{t}\right\} ; \\
R_{\phi}^{\phi}=R_{a b}\left(\partial_{\phi}\right)^{a} \nabla^{b} \phi=\nabla \cdot\left\{\nabla_{\mathrm{d} \phi^{\sharp}} \partial_{\phi}\right\} .
\end{gathered}
$$

Let the indices $A, B \in\{t, \phi\}$ then we can collect these results (four of them altogether) as

$$
R_{A}^{B}=\nabla \cdot\left\{\nabla_{\left(\mathrm{d} x^{B}\right)^{\sharp}} \partial_{A}\right\} .
$$

Unwrapping the covariant derivatives

$$
R_{A}^{B}=\frac{1}{\sqrt{-g_{4}}} \partial_{b}\left(\sqrt{-g_{4}} g^{B a} \Gamma_{a A}^{b}\right)
$$

If we now let the index $i$ range over every coordinate except the two Killing coordinates $t$ and $\phi$, then

$$
R_{A}^{B}=\frac{1}{\sqrt{-g_{4}}} \partial_{i}\left(\sqrt{-g_{4}} g^{B a} \Gamma_{a A}^{i}\right)
$$


Making this all very explicit, there are now four Landau-Lifshitz like results in total. They are:

$$
\begin{aligned}
R_{t}{ }^{t} & =\frac{1}{\sqrt{-g_{4}}} \partial_{i}\left(\sqrt{-g_{4}} g^{t a} \Gamma_{a t}^{i}\right) ; \\
R_{t}{ }^{\phi} & =\frac{1}{\sqrt{-g_{4}}} \partial_{i}\left(\sqrt{-g_{4}} g^{\phi a} \Gamma_{a t}^{i}\right) ; \\
R_{\phi}{ }^{t} & =\frac{1}{\sqrt{-g_{4}}} \partial_{i}\left(\sqrt{-g_{4}} g^{t a} \Gamma_{a \phi}^{i}\right) ; \\
R_{\phi}{ }^{\phi} & =\frac{1}{\sqrt{-g_{4}}} \partial_{i}\left(\sqrt{-g_{4}} g^{\phi a} \Gamma_{a \phi}^{i}\right) .
\end{aligned}
$$

Furthermore, recall that in stationary axisymmetric spacetimes we can always choose coordinates to block diagonalize the metric: $g_{a b}=g_{A B} \oplus g_{i j}$. But then

$$
g^{B a} \Gamma_{a A}^{i}=g^{B C} \Gamma_{C A}^{i}=g^{B C} g^{i j} \Gamma_{j C A}=-\frac{1}{2} g^{B C} g^{i j} \partial_{j} g_{C A} .
$$

So finally we have the relatively compact result

$$
R_{A}^{B}=-\frac{1}{2} \frac{1}{\sqrt{-g_{4}}} \partial_{i}\left(\sqrt{-g_{4}} g^{B C} g^{i j} \partial_{j} g_{C A}\right) \text {. }
$$

This can be rearranged in a number of different ways. As an illustration we point out

$$
\begin{aligned}
R_{A B}= & -\frac{1}{2} \frac{1}{\sqrt{-g_{4}}} \partial_{i}\left(\sqrt{-g_{4}} g^{i j} \partial_{j} g_{A B}\right) \\
& +\frac{1}{2} g^{i j} \partial_{i} g_{A C} g^{C D} \partial_{j} g_{D B} .
\end{aligned}
$$

We again see that our two-congruence extension of the Raychaudhuri equation has given us additional useful information regarding the Ricci tensor which might be difficult to extract by other means. 


\subsection{Discussion}

In this chapter we have developed several useful extensions of the usual Raychaudhuri equation. The main theme has been to relate various linear combinations of components of the Ricci tensor to divergences of suitably defined fluxes. We have worked with timelike congruences, spacelike congruences, and non-normalized congruences, in all cases being able to say just a little bit more (and sometimes much more) than the standard Raychaudhuri equation would imply. Furthermore, note that in Chapter 3 we have used a couple of the results from the time-like Raychaudhuri equation to construct different entropy bounds for uncollapsed matter.

One potentially far-reaching result is the "two congruence" extension of the Raychauduri equation presented in equations (4.70) and (4.71). 



\section{The quantum interest conjecture in $(3+1)$ dimensional Minkowski} space

Tt is well-known that quantum physics permits arbitrarily large negaLive energy densities at individual points [102, 103], though averages and integrated energies over volumes or lines are much more tightly constrained. This is of critical importance when developing singularity theorems, and other theorems based on global analysis, in that this simple observation is enough to guarantee that the so-called "classical energy conditions" are not fundamental physics; they are at best classical approximations to a more subtle quantum universe [104].

Note that without something similar to the energy conditions to constrain the spacetimes one wishes to consider as "physical", one can construct arbitrarily weird spacetimes containing such exotic objects as warpdrives [105, 106, 107, 108, 109], traversable wormholes [110, 111, 112, 113, 31, 114, 115, 116, 117, 118], singularity-free “black holes" [119], GNACHOs (gravitationally negative anomalous compact halo objects) [120], violations of the generalized second law [121], violations of cosmic censorship [122], and even time machines [123, 124, 125, 126, 127, 128]. Because 
of the need to keep such oddities somewhat constrained, and avoid a complete free-for-all, much work has gone into developing "averaged energy conditions" of various types [129, 130, 131, 132, 133], though even here there are issues associated with quantum field theory anomalies [134].

In particular, within the framework of semi-classical General Relativity (GR), the Quantum Inequalities (QIs) are an extremely important tool for constraining both negative energies, and more generally exotic phenomena which violate the classical energy conditions. Originally introduced by Ford [135], and subsequently extensively investigated by Ford and Roman [136, 137, 138, 139], and their collaborators [140, 141, 142, 143, 144], the QIs impose a lower bound for the integral of the renormalized stressenergy tensor along a suitable time interval, weighted by a suitable test function. Initially the test function was chosen to be a Lorentzian function [139], but subsequently Flanagan found a result independent of the specific choice of the test function in flat $(1+1)$ space-time [145]. With a distinct approach, Fewster and Eveson also obtained a bound independent of the weight function in 4 and 2 dimensional Minkowski space [146], though their result in 2 dimensions was somewhat weaker than that of Flanagan [145].

A related but distinct restriction for negative energies is imposed by the Quantum Interest Conjecture (QIC), introduced by Ford and Roman [147]. Informally speaking, the QIC states that "overall" the energy density must be positive, any negative energy density in one region must be overcompensated by positive energy in another region. When restricted to isolated pulses of energy, the QIC not only constrains the amount of negative energy in a pulse, but also the time interval between any negative energy pulse and the larger positive energy pulse that must also be present. The (positive) nett energy of the two pulses is called the Quantum Interest, and grows monotonically with the time separation. Initially Ford and Roman used delta function energy pulses to prove the conjecture, and it had not been generalized to arbitrarily shaped energy pulses in 4 dimensional 
flat space-time, nor was it initially formulated for curved space-time. A much more general approach to the QIC that is not restricted to isolated pulses was developed by Fewster and Teo in [148], wherein they created a Sobolev-space-based technical apparatus to reinterpret the QIs and the QIC as eigenvalue problems for a one-dimensional quantum mechanical pseudo-Hamiltonian. This technique relates the $(1+1)$ dimensional QIs and QIC to the nonexistence of bound states for the Schrödinger equation (SDE) in one dimension, while the $(3+1)$ dimensional QIs and QIC are related to the nonexistence bound states for the bi-harmonic Schrödinger equation (bSDE). Within this framework, Fewster and Teo [148], and later Teo and Wong [149], were able to prove the QIC in $(1+1)$ dimensional Minkowski space; however they could not extend their proof to the $(3+1)$ dimensional case. In Teo and Wong's approach [149] critical use is made of a theorem by Simon [150], regarding the existence of bound states for the 1 dimensional SDE. That is, Simon's theorem is used to prove a version of the QIC in $(1+1)$ dimensions, but the generalization to $(3+1)$ dimensions is troublesome.

Furthermore, in [151] Pretorius found an upper bound for the separation between two general energy pulses by applying a scaling argument to the test function. With this strategy, Pretorius also showed that the Quantum Interest grows almost linearly as the pulse separation increases, which is the same result obtained in [147].

Using the approach explained in [148], we shall provide a simple variational proof for a variant of the QIC in 4 dimensional flat space-time, (and in fact in any even dimensional spacetime), by proving the equivalent of Simon's theorem for the bi-harmonic Schrödinger equation (and more generally for the multi-harmonic Schrödinger equation). We shall do this via a variational argument (the Rayleigh-Ritz bound on the lowest eigenvalue) coupled with a power series expansion of the test function used in the related one-dimensional pseudo-Hamiltonian problem. 


\subsection{Quantum Inequalities and the Quantum in- terest Conjecture}

\subsubsection{The Quantum Inequalities}

The Quantum Inequalities give a lower bound for the expectation value of the renormalized stress-energy tensor (in a quantum state $\psi$ ) when evaluated along a complete timelike geodesic,

$$
\begin{aligned}
I_{\psi, w} & \equiv \int_{-\infty}^{\infty}\left\langle T_{00}^{r e n}(t, 0)\right\rangle_{\psi} w(t) \mathrm{d} t \\
& \equiv \oint\left\langle T_{00}^{r e n}(t, 0)\right\rangle_{\psi} w(t) \mathrm{d} t .
\end{aligned}
$$

Here $w(t)$ is a more-or-less freely-specifiable test function (weighting function), which is non-negative and integrates to unity: $\int_{-\infty}^{+\infty} w(x) \mathrm{d} x=1$. Initially, the stress-tensor is treated in the test-field limit, so the background is simply taken to be Minkowski space, and the timelike geodesic is specified by the 4 -velocity $V^{a}=(1 ; \overrightarrow{0})$. Since our integrals almost always run from $-\infty$ to $+\infty$ we shall for brevity often use the shorthand symbol $\oint=\int_{-\infty}^{+\infty}$.

In their initial analysis, Ford and Roman used a Lorentzian test function to yield to a lower bound which depend on modified Bessel functions [139]. However a more general inequality was found by Flanagan in $(1+1)$ dimensional flat space-time [145], where he used Quantum Field Theory for a massless scalar field minimally coupled to gravity to find the optimum lower bound independent of the specific choice of the test function,

$$
I_{\psi, w} \geq-\frac{1}{24 \pi} \oint \frac{\left(w^{\prime}(t)\right)^{2}}{w(t)} \mathrm{d} t .
$$

Fewster and Eveson used a distinct approach [146] to yield inequalities in (1+1) dimensions,

$$
I_{\psi, w} \geq-\frac{1}{16 \pi} \oint \frac{\left(w^{\prime}(t)\right)^{2}}{w(t)} \mathrm{d} t
$$


and in $(3+1)$ dimensions,

$$
I_{\psi, w} \geq-\frac{1}{16 \pi} \oint\left(\left[w^{1 / 2}\right]^{\prime \prime}(t)\right)^{2} \mathrm{~d} t .
$$

It is obvious that the better bound in $(1+1)$ dimensions is given by Flanagan's result rather than the one by Fewster and Eveson. However the most general and optimum bound in $(3+1)$ dimensional flat space-time, for a massless scalar field minimally coupled to gravity, is shown in equation (5.5).

Combining the results of Flanagan, and Fewster and Eveson, it is possible to rewrite the QIs in 2m-dimensional spacetime in the more general form [148], (writing $w(t)=f(t)^{2}$ to automatically enforce the positivity constraint),

$$
\oint\left\langle T_{00}^{r e n}(t, 0)\right\rangle_{\psi}[f(t)]^{2} \mathrm{~d} t \geq-\frac{1}{c_{m}} \oint\left[D^{m} f(t)\right]^{2} \mathrm{~d} t,
$$

where $D$ is the derivative operator, and the various different constants are given by

$$
c_{m}=\left\{\begin{array}{cc}
6 \pi & m=1 ; \\
m \pi^{m-1 / 2} 2^{2 m} \Gamma\left(m-\frac{1}{2}\right) & m \geq 2 ;
\end{array}\right.
$$

and where the weighting function is now normalized to $\int_{-\infty}^{+\infty}|f(x)|^{2} \mathrm{~d} x=$ 1. The case $m=1$ is equivalent to equation (5.3), while equation (5.5) is equivalent to $m=2$. After integrating equation (5.6) by parts, the QIs become a statement regarding the non-existence of negative eigenvalues for a one-dimensional pseudo-Hamiltonian,

$$
\langle f|H| f\rangle \geq 0
$$

where,

$$
H=(-1)^{m} D^{2 m}+c_{m}\left\langle T_{00}^{r e n}(t, 0)\right\rangle_{\psi} .
$$

The quantity $c_{m}\left\langle T_{00}^{r e n}(t, 0)\right\rangle_{\psi}$ can effectively be viewed as an "potential" $V$ for a quantum mechanical system,

$$
V \equiv c_{m}\left\langle T_{00}^{r e n}(t, 0)\right\rangle_{\psi}
$$


and the QIs become the statement

$$
\left\langle f\left|\left\{(-1)^{m} D^{2 m}+V\right\}\right| f\right\rangle \geq 0,
$$

We shall now use the framework of quantum mechanics to analyze the QIs and the QIC as an eigenvalue problem.

\subsubsection{The QIC as an eigenvalue problem}

As adopting this point of view allows us to use the mathematical background of one-dimensional quantum mechanics, we will change to the standard quantum mechanical notation. For instance, the differential operator (5.9) becomes the quantum pseudo-Hamiltonian

$$
H=P^{2 m}+V
$$

where $P$ and $V$ are operators on the usual Hilbert space of square-integrable functions. The eigenvalue problem for this Hamiltonian, in coordinates, is the ODE [ordinary differential equation] (we have set $\hbar / 2 m \rightarrow 1$ to simplify the algebra),

$$
(-1)^{m} \frac{\mathrm{d}^{2 m}}{\mathrm{~d} x^{2 m}} \varphi(x)+V(x) \varphi(x)=E \varphi(x),
$$

for the eigenfunctions $\varphi(x)$ which again belong to the usual Hilbert space of square-integrable functions. (If desired, a more rigorous approach can be followed, as in [148]). Taking $m=1$, (i.e., (1+1) spacetime dimensions), we recover the one-dimensional time-independent Schrödinger equation (SDE)

$$
-\frac{\mathrm{d}^{2}}{\mathrm{~d} x^{2}} \varphi(x)+V(x) \varphi(x)=E \varphi(x),
$$

while, for $m=2$, (i.e., $(3+1)$ spacetime dimensions), we obtain the onedimensional time-independent bi-harmonic Schrödinger Equation (bSDE),

$$
\frac{\mathrm{d}^{4}}{\mathrm{~d} x^{4}} \varphi(x)+V(x) \varphi(x)=E \varphi(x)
$$


For the case of the SDE, there is a theorem by Simon [150] which, by imposing a constraint on the potential, ensures the existence of a bound state for the Hamiltonian. In a simple form it reads:

\section{Simon's Theorem:}

Let $V(x)$ obey $\int_{-\infty}^{\infty}\left(1+x^{2}\right)|V(x)| d x<\infty$, with $V(x)$ not zero almost everywhere. Then $H=-\mathrm{d}^{2} / \mathrm{d} x^{2}+V(x)$ has a negative eigenvalue if

$$
\int_{-\infty}^{\infty} V(x) \mathrm{d} x \leq 0
$$

With the help of this theorem, it is possible to generalize the QIC to a wider set of energy pulses, and not only be restricted to $\delta$-function pulses as in [147].

In order to better understand the QIC in this framework, first we have to point out that a potential which fulfills the QIs must violate condition (5.16). Now let us rewrite the potential as

$$
V(x)=V(x)_{+}-V(x)_{-} .
$$

This splits the potential into its positive part minus its negative contributions, with $V(x)_{ \pm} \geq 0$. Then, if the potential violates condition (5.16), from (5.17) we have

$$
\oint V(x)_{+} \mathrm{d} x>\oint V(x)_{-} \mathrm{d} x .
$$

This means, if we go back to the GR terminology, that the QIs imply that the expectation value of the renormalized stress-energy tensor must satisfy

$$
\oint\left\langle T_{00}^{r e n}(t, 0)\right\rangle_{\psi} \mathrm{d} t>0
$$

which is slightly stronger than the averaged weak energy condition (AWEC). Splitting the energy density into its positive and negative parts

$$
\left\langle T_{00}^{r e n}(t, 0)\right\rangle=\left\langle T_{00}^{r e n}(t, 0)\right\rangle_{+}-\left\langle T_{00}^{r e n}(t, 0)\right\rangle_{-},
$$


this implies

$$
\oint\left\langle T_{00}^{r e n}(t, 0)\right\rangle_{+} \mathrm{d} x>\oint\left\langle T_{00}^{r e n}(t, 0)\right\rangle_{-} \mathrm{d} x .
$$

That is, the positive part of a stress tensor that satisfies the QIs, must always overcompensate its negative part, this is one version of QIC. (Of course, there are significant elements missing from this form of the conjecture, as compared with the original form and Pretorius' scaling argument [151]. Specifically there is no immediate way to extract a bound for the time separation between the energy pulses and the Quantum Interest as a function of this separation. However, a benefit of the current discussion is that we are not limited to delta function pulses of pulses of compact support.)

In the next section we will prove Simon's theorem for the SDE by expanding an appropriate class of test functions in a power series; this strategy will be used again for the bSDE in section 4, and in the appendix a similar result will be obtained in arbitrary even-dimensional Minkowski spacetime.

\subsection{1+1 dimensional Minkowski space}

In order to prove Simon's theorem, avoiding most of the technical issues arising in the formal proof [150], and keeping in mind the idea of generalizing the theorem to the bSDE, and even higher-derivative "Hamiltonians", we shall use a power series expansion of the test function in the SDE to find the conditions for the potential $V$ to bind.

\subsubsection{Gaussian wave-function}

Let us start, for simplicity and clarity, with a Gaussian test function,

$$
\varphi_{\text {test }}=\left[\frac{\exp \left\{-(x-\mu)^{2} /\left(2 \sigma^{2}\right)\right\}}{\sqrt{2 \pi} \sigma}\right]^{1 / 2},
$$


which automatically enforces the unit normalization $\oint|\varphi(x)|^{2} \mathrm{~d} x=1$. Therefore, a variational argument implies that for the lowest eigenvalue of the SDE one has:

$$
E \leq \oint\left[\varphi^{\prime}(x)^{2}+V(x) \varphi(x)^{2}\right] \mathrm{d} x
$$

Then the kinetic term is $\oint \varphi^{\prime}(x)^{2}=1 / \sigma^{2}$, whereas (assuming all the moments $\left|\oint x^{2 n} V(x) d x\right|<\infty$ so that the expansion makes sense)

$$
\begin{aligned}
& \oint V(x) \varphi(x)^{2} \mathrm{~d} x= \\
& \frac{1}{\sqrt{2 \pi} \sigma} \sum_{n=0}^{\infty} \frac{(-1)^{n}}{n !\left(2 \sigma^{2}\right)^{n}} \times \oint V(x)(x-\mu)^{2 n} \mathrm{~d} x .
\end{aligned}
$$

Interchanging the integral and the summation is justified since the Gaussian function $\varphi(x)^{2}$ is an analytic function of $x$ whose Taylor series has an infinite radius of convergence.

For a fixed $\mu$ we now see that:

$$
E \sigma^{2} \leq 1+\frac{\sigma}{\sqrt{2 \pi}} \oint V(x) \mathrm{d} x+O(1 / \sigma)
$$

From this last expression, we can see that if

$$
\oint V(x) \mathrm{d} x<0
$$

then for $\sigma$ sufficiently large we can guarantee $E \sigma^{2}<0$, (hence $E<0$ and the potential will bind, and so violate the QIs). This is close to Simon's condition. (We currently have a $<$, rather than Simon's $\leq$. The only "difficult" thing about Simon's theorem is proving the existence of a bound state in the marginal = case. Note that the weaker result we have here is already enough, in GR language, to assure that the stress tensor satisfies the AWEC.)

\subsubsection{Generic class of test functions}

To deal with the marginal case, we proceed by using the fact that we are free to choose the test function in a more-or-less arbitrary manner, and so 
to use this freedom obtain tighter constraints. Let us start by picking some function $g(x)$ that is analytic on the entire real line (infinite radius of convergence) and then constructing the piecewise analytic function

$$
h(x)=g(|x|) .
$$

Note that $h(x)$ need not, and typically will not, be analytic at zero. We then enforce the normalization

$$
\oint h(x) \mathrm{d} x=1, \quad \oint x h(x) \mathrm{d} x=0, \quad \oint x^{2} h(x) \mathrm{d} x=1 .
$$

Suitable examples of such functions are

$$
h(x)=\frac{\exp (-\sqrt{2}|x|)}{\sqrt{2}}
$$

and

$$
h(x)=\sum_{i=1}^{N} c_{i} \exp \left(-|x| / d_{i}\right)
$$

subject to

$$
2 \sum_{i=1}^{N} c_{i} d_{i}=1 ; \quad 4 \sum_{i=1}^{N} c_{i} d_{i}^{3}=1 .
$$

We now choose

$$
\varphi_{\text {test }}=\sqrt{\frac{h([x-\mu] / \sigma)}{\sigma}}=\sqrt{\frac{g(|x-\mu| / \sigma)}{\sigma}},
$$

whence

$$
\oint|\varphi(x)|^{2} \mathrm{~d} x=1, \quad \oint x|\varphi(x)|^{2} \mathrm{~d} x=\mu
$$

and

$$
\oint(x-\mu)^{2}|\varphi(x)|^{2} \mathrm{~d} x=\sigma^{2} .
$$

Moreover, $\oint \varphi^{\prime}(x)^{2} d x=\kappa / \sigma^{2}$, with

$$
\kappa \equiv \frac{1}{4} \oint \frac{h^{\prime}(x)^{2}}{h(x)} \mathrm{d} x .
$$


The numeric value of $\kappa$ depends on the choice of the test function and further optimizations may be useful. For now, let us focus on the Taylor expansion of $g(x)$ :

$$
g(x)=\sum_{n=0}^{\infty} a_{n} x^{n} .
$$

This is assumed to exist (by analyticity), and to converge on the entire real line. We now deduce the existence of a similar power series expansion for $h(x)$ :

$$
h(x)=g(|x|)=\sum_{n=0}^{\infty} a_{n}|x|^{n} .
$$

This is now an expansion in the variable $|x|$. Since it depends on $|x|$, this is not a Taylor series for $h(x)$, but by construction it is convergent over the entire real line. Then for the shifted and rescaled test function we have

$$
\left|\varphi_{\text {test }}\right|^{2}=\frac{1}{\sigma} \sum_{n=0}^{\infty} a_{n}\left[\frac{|x-\mu|}{\sigma}\right]^{n} .
$$

Assuming all the moments $\left.|\oint| x\right|^{n} V(x) \mathrm{d} x \mid<\infty$, so that the expansion makes sense, we furthermore have

$$
E \leq \frac{\kappa}{\sigma^{2}}+\frac{1}{\sigma} \sum_{n=0}^{\infty} \frac{a_{n}}{\sigma^{n}} \oint V(x)|x-\mu|^{n} \mathrm{~d} x .
$$

In interchanging the summation and the limit we have first split $\oint \equiv$ $\int_{-\infty}^{+\infty}=\int_{-\infty}^{\mu}+\int_{\mu}^{+\infty}$, and then appealed to the analyticity of $g(x)$ and $g(-x)$ on the appropriate ranges, finally recombining the two sets of integrals to run over the entire real line.

We now have the potential for additional relevant terms to appear in the series expansion for the variational bound on the lowest eigenvalue $E$, and is convenient to place some restrictions on the coefficients $a_{n}$ appearing in the series expansion.

First, if $a_{0}=0$, then

$$
h(x) \sim a_{1}|x|+\ldots,
$$


so that

$$
\frac{\left[h^{\prime}(x)\right]^{2}}{h(x)} \sim \frac{a_{1}}{|x|}+\ldots
$$

Thus in order for the integral defining $\kappa$ [equation (5.35)] to converge at $x=0$ it is necessary to chose $a_{1}=0$. So in fact

$$
h(x) \sim a_{2} x^{2}+\ldots,
$$

and

$$
\frac{\left[h^{\prime}(x)\right]^{2}}{h(x)} \sim 4 a_{2}+\ldots
$$

In this situation we then cannot extract much information, since the SDE now merely gives

$$
E \sigma^{2} \leq \kappa+O\left(\sigma^{-1}\right)
$$

Hence, to extract useful information, we need $a_{0} \neq 0$, and so (since probability densities are always positive), $a_{0}>0$.

In contrast, for $a_{0}>0$, the integral defining $\kappa$ [equation (5.35)] always converges at $x=0$, since

$$
h(x) \sim a_{0}+a_{1}|x|+\cdots,
$$

and so

$$
\frac{\left[h^{\prime}(x)\right]^{2}}{h(x)} \sim \frac{a_{1}^{2}}{a_{0}}+\ldots
$$

Thus we have

$$
E \sigma^{2} \leq \kappa+\sigma a_{0} \oint V(x) \mathrm{d} x+a_{1} \oint|x-\mu| V(x) \mathrm{d} x+O(1 / \sigma) .
$$

Therefore, with $\sigma$ sufficiently large the only important contributions come from the first two terms ( $n=0$, and $n=1$ ) of the power series expansion.

Here, as for the Gaussian test functions, the condition $\oint V(x) \mathrm{d} x<0$ implies binding; which means that the expectation value of the renormalized stress-energy tensor must fulfill the AWEC. However, if we consider 
the borderline of AWEC violation, i.e., $\oint V(x) \mathrm{d} x=0$, then the next term in the expansion is now no longer neglectable:

$$
E \sigma^{2} \leq \kappa+a_{1} \oint|x-\mu| V(x) \mathrm{d} x+O(1 / \sigma) .
$$

But recall that $a_{1}$ is completely arbitrary, both in sign and in magnitude. That is, if for any value of $\mu$

$$
\oint|x-\mu| V(x) \mathrm{d} x \neq 0,
$$

then this implies the existence of a bound state for the SDE. This is a very strong constraint on the potential and it quickly yields a proof of Simon's theorem. Taking the converse of the above, if $\oint V(x) \mathrm{d} x=0$ then to prevent the occurrence of a bound state we must have

$$
\forall \mu: \quad \oint|x-\mu| V(x) \mathrm{d} x=0 .
$$

Differentiating twice with respect to $\mu$ we get

$$
\begin{array}{r}
\forall \mu: \quad 2 \oint \delta(x-\mu) V(x) d x=0 \\
\Rightarrow V(\mu)=0 .
\end{array}
$$

Thus any non-zero potential binds if its integral is null. Combining this with the fact that $\oint V(x) d x<0$ also implies binding, now provides a simple variational proof of Simon's theorem, equation (5.16), though under the much stronger technical conditions that all the moments exist,

$$
\left.|\oint| x\right|^{n} V(x) \mathrm{d} x \mid<\infty
$$

It is these stronger technical conditions - which are still physically quite reasonable and are certainly satisfied by isolated pulses of stress energy that will make it easy for us to extend Simon's theorem to the bi-harmonic Schrödinger equation, and so lead to a $(3+1)$ dimensional version of the QIC. 


\subsection{3+1 dimensional Minkowski space}

To generalize the condition given by Simon's theorem to the bSDE, we proceed as before, expanding appropriate test functions in power series. Applying a variational argument to the bSDE we find that the lowest eigenvalue satisfies

$$
E \leq \oint\left[\varphi^{\prime \prime}(x)^{2}+V(x) \varphi(x)^{2}\right] \mathrm{d} x
$$

assuming that the test functions are normalized. We now use this relation to probe for the existence of a bound state for the pseudo-Hamiltonian for the bSDE, which is ultimately related to the 4 dimensional flat space-time QIs and the QIC.

\subsubsection{Gaussian wave-function}

We start with the normalized Gaussian test function previously used for the SDE, equation (5.22). The kinematic term yields $\oint \varphi^{\prime \prime}(x)^{2} \mathrm{~d} x=\kappa^{2} / \sigma^{4}$, with $\kappa$ a numeric constant. Assuming all appropriate moments exist, we again expand the test function in a power series, whence

$$
\begin{aligned}
& \oint V(x) \varphi^{2}(x) d x= \\
& \frac{1}{\sqrt{2 \pi} \sigma} \sum_{n=0}^{\infty} \frac{(-1)^{n}}{n !\left(2 \sigma^{2}\right)^{n}} \times \oint V(x)(x-\mu)^{2 n} \mathrm{~d} x .
\end{aligned}
$$

Then, from (5.53)

$$
\begin{aligned}
E \sigma^{4} \leq & \kappa^{2}+\frac{\sigma^{3}}{\sqrt{2 \pi}} \oint V(x) \mathrm{d} x \\
& -\frac{\sigma}{2 \sqrt{2 \pi}} \oint V(x)(x-\mu)^{2} \mathrm{~d} x+O(1 / \sigma) .
\end{aligned}
$$

As for the SDE, in this case also $\oint V(x) \mathrm{d} x<0$ implies that the potential binds for a sufficiently large $\sigma$. This gives us most of Simon's theorem, apart from the extremal case where the integral vanishes. 
However, even with the Gaussian test function, in this bSDE case we recover more information than for the SDE. Specifically if $\oint V(x) \mathrm{d} x=0$, then the next term of the expansion is important, and to guarantee the absence of a bound state we must also enforce:

$$
\forall \mu: \quad \oint(x-\mu)^{2} V(x) \mathrm{d} x \leq 0 .
$$

This constraint, while certainly significant, is not quite strong enough to imply Simon's theorem.

\subsubsection{Generic class of test functions}

Finally, for the bSDE, we consider the same normalization, (5.28), and the same choice for the generic test function, as we used for the SDE, (5.32). Note

$$
\oint \varphi^{\prime \prime}(x)^{2} \mathrm{~d} x=\frac{\kappa}{\sigma^{4}}
$$

with

$$
\begin{aligned}
\kappa & \equiv \oint\left\{[\sqrt{h}]^{\prime \prime}(x)\right\}^{2} \mathrm{~d} x \\
& =\frac{1}{16} \oint \frac{\left[2 h(x) h^{\prime \prime}(x)-h^{\prime}(x)^{2}\right]^{2}}{h(x)^{3}} \mathrm{~d} x .
\end{aligned}
$$

In view of the fact that we have chosen $h(x)=g(|x|)$ this becomes (note the delta function arising from twice differentiating the absolute value)

$$
\begin{aligned}
& \kappa \equiv \frac{1}{16} \times \\
& \oint \frac{\left\{2 g(|x|)\left[g^{\prime \prime}(|x|)+g^{\prime}(|x|) \delta(x)\right]-g^{\prime}(|x|)^{2}\right\}^{2}}{g(|x|)^{3}} \mathrm{~d} x .
\end{aligned}
$$

In order to derive a useful bound we will want $\kappa$ to be finite, which means that we want the coefficient of the delta function to be zero, that is $a_{1}=0$. To make the integral converge at zero we also want $a_{0} \neq 0$, and in fact 
must have $a_{0}>0$. In contrast, $a_{2}$ and $a_{3}$ are unconstrained as to sign and magnitude and we have

$$
\begin{aligned}
E \sigma^{4} & \leq \kappa+a_{0} \sigma^{3} \oint V(x) \mathrm{d} x \\
& +a_{2} \sigma \oint V(x)|x-\mu|^{2} \mathrm{~d} x \\
& +a_{3} \oint V(x)|x-\mu|^{3} \mathrm{~d} x+O(1 / \sigma) .
\end{aligned}
$$

As before, by suitably choosing a sufficiently large $\sigma$, we see that the condition $\oint V(x) \mathrm{d} x<0$ implies binding.

To extract further information from (5.61) it is necessary to check what happens if we set $\oint V(x) \mathrm{d} x=0$. Thereafter the next two terms in the expansion become relevant. However both the sign and magnitude of $a_{2}$ and $a_{3}$ are freely specifiable, hence (for any $\mu$ ) either

$$
\oint|x-\mu|^{2} V(x) \mathrm{d} x \neq 0
$$

or

$$
\oint|x-\mu|^{3} V(x) \mathrm{d} x \neq 0
$$

is a sufficient condition for the Hamiltonian to have a bound state.

Conversely, if $\oint V(x) \mathrm{d} x=0$, a necessary condition for the absence of a bound state is that

$$
\forall \mu: \quad \oint|x-\mu|^{2} V(x) \mathrm{d} x=0,
$$

and

$$
\forall \mu: \quad \oint|x-\mu|^{3} V(x) \mathrm{d} x=0 .
$$

Now if we differentiate the last expression with respect to $\mu$ then

$$
\forall \mu: \quad \oint|x-\mu|^{2} \operatorname{sign}(x-\mu) V(x) \mathrm{d} x=0,
$$

whence, combining with Eq. (5.64), we have

$$
\forall \mu: \quad \int_{\mu}^{\infty}|x-\mu|^{2} V(x) \mathrm{d} x=0 .
$$


If we repeatedly differentiate the last expression with respect to $\mu$ then

$$
\begin{gathered}
\forall \mu: \quad \int_{\mu}^{\infty}|x-\mu| V(x) \mathrm{d} x=0 . \\
\forall \mu: \quad \int_{\mu}^{\infty} V(x) \mathrm{d} x=0 .
\end{gathered}
$$

and finally $V(x)=0$. That is, if $\oint V(x) \mathrm{d} x=0$, a necessary condition for the absence of a bound state is that $V(x)=0$. Conversely if $\oint V(x) \mathrm{d} x=0$ and $V(x) \not \equiv 0$, then this is a sufficient condition for the presence of a bound state. This proves the equivalent of Simon's theorem for the 4th-order bSDE and, furthermore proves the QIC in $(3+1)$ dimensional Minkowski space.

Note that the version of the QIC that we have proved is this: The QI's imply that either $\left\langle T_{00}^{r e n}(t, 0)\right\rangle \equiv 0$ everywhere along the world-line, or

$$
\oint\left\langle T_{00}^{r e n}(t, 0)\right\rangle_{\psi} \mathrm{d} t>0
$$

which we emphasize is slightly stronger than the AWEC. Splitting the energy density into its positive and negative parts, this implies that as long as $\left\langle T_{00}^{r e n}(t, 0)\right\rangle$ is not identically zero along the world line, then

$$
\left.\oint\left\langle T_{00}^{r e n}(t, 0)\right\rangle_{+} \mathrm{d} x\right\rangle \oint\left\langle T_{00}^{r e n}(t, 0)\right\rangle_{-} \mathrm{d} x .
$$

That is, any negative energy "loan" is overcompensated elsewhere along the world line.

\subsection{Higher dimensions}

Having now seen the argument in $(1+1)$ and $(3+1)$ dimensions it is clear how to generalize to any even number of dimensions. Consider a $2 m$ dimensional (that is, $([2 m-1]+1)$ dimensional) spacetime. Then the QIs are equivalent to the statement:

$$
\nexists \text { bound state : } \quad H=(-1)^{m} \frac{\mathrm{d}^{2 m}}{\mathrm{~d} x^{2 m}}+V(x) .
$$


Picking the generic class of test functions discussed previously, and applying a variational argument, the ground state energy is bounded by

$$
E \leq \oint\left\{\left[\varphi^{(m)}(x)\right]^{2}+V(x)[\varphi(x)]^{2}\right\} \mathrm{d} x .
$$

The kinetic term is

$$
\oint\left[\varphi^{(m)}(x)\right]^{2} \mathrm{~d} x=\frac{\kappa^{2}}{\sigma^{2 m}}
$$

(where the precise numerical value of $\kappa$ is not important as long as it is finite), whereas (assuming all appropriate moments exist)

$$
\oint V\left[\varphi^{2}\right] \mathrm{d} x=\frac{1}{\sigma} \sum_{n=0}^{\infty} \frac{a_{n}}{\sigma^{n}} \times \oint V(x)|x-\mu|^{n} \mathrm{~d} x .
$$

The interchange of the summation and integration is again justified by the assumed piecewise analytic nature of the test function. Therefore

$$
\begin{aligned}
E \sigma^{2 m} \leq & \kappa^{2}+\sigma^{2 m-1} \sum_{n=0}^{2 m-1} \frac{a_{n}}{\sigma^{n}} \times \oint V(x)|x-\mu|^{n} \mathrm{~d} x \\
& +O(1 / \sigma),
\end{aligned}
$$

where there will be some set of constraints on the coefficients $a_{n}$ to keep $\kappa$ finite.

Now since $\varphi(x)$ is a test function we are always free to choose $a_{0} \neq 0$, and since probability densities are always positive, this forces $a_{0}>0$. We are also free to (temporarily) choose all the odd $a_{2 n+1}=0$, which is a convenience (since it implies the absence of squared delta functions coming from differentiations of the absolute value function) to guarantee $\kappa$ finite. Then

$$
\begin{aligned}
E \sigma^{2 m} \leq & \kappa^{2}+\sigma^{2 m-1} \sum_{n=0}^{m-1} \frac{a_{2 n}}{\sigma^{2 n}} \times \oint V(x)|x-\mu|^{2 n} \mathrm{~d} x \\
& +O(1 / \sigma),
\end{aligned}
$$

But then if $\oint V(x) \mathrm{d} x<0$, it follows that for $\sigma$ sufficiently large we can guarantee $E \sigma^{2 m}<0$, whence $E<0$, and the potential will bind, (thus violating the QIs). This is the easy bit. 
- In particular, $\nexists$ bound state $\Longrightarrow \oint V(x) \mathrm{d} x \geq 0$.

Now consider the borderline case $\oint V(x) \mathrm{d} x=0$. We are again free to choose $a_{0} \neq 0$ (and so $a_{0}>0$ ), and are also free to choose both the sign and magnitude of all the $a_{2 n}$. But this implies the potential will bind unless

$$
\forall \mu: \quad \oint V(x)|x-\mu|^{2 n} \mathrm{~d} x=0 ; \quad \forall n \in(0, m-1) .
$$

In particular

$$
\forall \mu: \quad \oint V(x)|x-\mu|^{2 m-2} \mathrm{~d} x=0 .
$$

Now "turn on" one of the odd $a_{2 n+1}$. Specifically, consider $a_{2 m-1}$. Since for $m \geq 2$ we have

$$
\frac{\mathrm{d}^{m}}{\mathrm{~d} x^{m}}\left(|x|^{2 m-1}\right)=0
$$

we can switch on this $a_{2 m-1}$ coefficient without risk of developing a squared delta function in the evaluation of $\kappa$, and so keep $\kappa$ finite. But then since $a_{2 m-1}$ is arbitrary as to sign and magnitude, to prevent binding we must have

$$
\forall \mu: \quad \oint V(x)|x-\mu|^{2 m-1} \mathrm{~d} x=0 .
$$

Differentiating with respect to $\mu$

$$
\forall \mu: \quad \oint V(x)|x-\mu|^{2 m-2} \operatorname{sign}(x-\mu) \mathrm{d} x=0,
$$

whence, combining the two preceeding equations, we see

$$
\forall \mu: \quad \int_{\mu}^{\infty} V(x)|x-\mu|^{2 m-2} \mathrm{~d} x=0 .
$$

Repeated differentiations with respect to $\mu$ will now eventually yield $V(x)=$ 0 .

That is, if $\oint V(x) \mathrm{d} x=0$ then to prevent a bound state we must have $V(x)=0$. Conversely, if $\oint V(x) \mathrm{d} x=0$ and $V(x) \not \equiv 0$, then there will be a bound state. 
- In particular, $\nexists$ bound state $\Longrightarrow$ either $V(x) \equiv 0$ or $\oint V(x) \mathrm{d} x>0$.

This now is a version of Simon's theorem appropriate to the multi-harmonic SDE, leading to a general even-dimensional version of the QIC: Either $\left\langle T_{00}^{r e n}(t, 0)\right\rangle \equiv 0$ everywhere along the world-line, or

$$
\oint\left\langle T_{00}^{r e n}(t, 0)\right\rangle_{\psi} \mathrm{d} t>0
$$

this last inequality being slightly stronger than the AWEC.

\subsection{Discussion}

With the variant of Simon's theorem that we have now proved for the bSDE, it is possible to reformulate the QIC for a more general range of energy pulses in $(3+1)$ dimensional flat space-time, as has been already done for the $(1+1)$ dimensional case. In flat space-time, an energy pulse which satisfies the QIs (and so the version of the QIC discussed above), must also fulfill an AWEC-like inequality. That is, the expectation value of the renormalized stress-energy tensor shall violate the condition provided by equation (5.16), and so satisfy (5.70).

Moreover, using the argument derived from equation (5.20), the QIC can be extended to $(3+1)$ dimensional Minkowski space. Furthermore, from (5.21) and (5.71), we know that the positive contributions of the expectation value of the renormalized stress-energy tensor must overcompesate its negative parts. The key trade-off in the current argument is that while we have been able to deduce a general result for arbitrary even dimensional Minkowski space, and while we are not limited to deltafunction pulses or pulses of compact support, we have on the other hand lost some of the precision information that can be deduced when stronger assumptions are made regarding the temporal distribution of the stress energy. 
Furthermore, this proof of the QIC that we have given in flat four dimensional space-time gives only a partial picture of the nature of negative energies and the constraints that can be placed on them, as it does not (yet) include the effects of curved spacetime. Indeed, for many technical reasons it would be preferable to work with the null energy condition (NEC), rather than the weak energy condition [130, 131, 132]. That is: A truly complete formulation of the QIs and QIC should really include curved spacetimes, at the very least $(3+1)$ dimensional curved spacetimes, and work with some version of the null energy condition - this is the key arena wherein the possibility of exotic phenomena such as traversable wormholes, warp-drives, and time machines are related to the existence of negative energies and the constraints thereupon. 



\section{6}

Conclusions

$\mathrm{T}$

The results developed in this thesis summarize work done in several different, but related topics in General Relativity. They all represent improvements on different aspects of the theory, from the geometrically fundamental analysis using the Raychaudhuri equation, to the emergence of quantum field theory in curved spacetime. However, these results will prove their importance insofar as they prove to be useful.

First, we use the Kodama vector to build a preferred time coordinate, and ultimately a geometrically preferred coordinate system. Furthermore, we have not only constructed such coordinate system, but also we have generalized Kodama's conservation law. This was done by appealing to the "warp product" nature of spherical symmetry. The coordinate system built has already been used to study Hawking radiation in [82, 83].

Furthermore, the entropy bounds derived in Chapter 3 demonstrate how it is possible to construct entropy bounds from very basic physics, with a minimum resort to quantum field theory in curved space time. Although this minimalist approach allowed us to bound the quasi-local entropy of two different spacetimes, the resulting bounds are still weaker than the more traditional bounds found in the literature. The main reason for this discrepancy is because we were considering horizonless objects only, i.e., uncollapsed matter such as a star, planet, monster, gravastar, black star, or quasi-black hole. 
We have also somewhat generalized the notion of surface gravity, in two different ways. In Chapter 2 we did so by considering the ingoing and outgoing null geodesics. On the other hand, in Chapter 3 the surface gravity was generalized to a three-vector, and ultimately it was its averaged version that was used to obtain the entropy bound for the static horizonless spacetime.

We then developed a series of extensions to the Raychaudhuri equation by considering different types of congruences, and spacetime symmetries. We have even constructed a "two congruence" version of the Raychaudhuri equation, which leads to perhaps somewhat unexpected axially symmetric Landau-Lifshitz like results.

Some of the generalizations to the Raychaudhuri equation, presented in Chapter 4, already proved useful when dealing with the entropy bounds of uncollapsed matter, as shown in Chapter 3. Specifically, equation (4.31) is used in the derivation of an entropy bound for static uncollapsed spacetimes. Similarly, the non-geodesic timelike Raychaudhuri equation for stationary axisymmetric spacetimes, equation (4.41), is used in the intermediate stages of the calculation of the entropy bound for an uncollapsed rotating body.

Lastly, we have proved a version of the Quantum Interest Conjecture, which provides a sort of restriction on the amount and duration of negative energy fluxes, and the exotic phenomena that is related to it. Of course, this proof of the Quantum Interest Conjecture in flat spacetime is only a partial picture of the whole negative energy associated phenomena. There are still several open questions regarding such phenomena, including any possible generalizations of the singularity theorems that include the behavior of quantized matter. 
Appendices 



\section{A}

Publications

\section{A.1 Quantum interest in $(3+1)$ dimensional Minkowski space}

$\mathrm{T}$

The so-called "Quantum Inequalities", and the "Quantum Interest Conjecture", use quantum field theory to impose significant restrictions on the temporal distribution of the energy density measured by a timelike observer, potentially preventing the existence of exotic phenomena such as "Alcubierre warp-drives" or "traversable wormholes". Both the quantum inequalities and the quantum interest conjecture can be reduced to statements concerning the existence or non-existence of bound states for a certain one-dimensional quantum mechanical pseudo-Hamiltonian. Using this approach, we shall provide a simple variational proof of one version of the Quantum Interest Conjecture in $(3+1)$ dimensional Minkowski space.

Phys. Rev. D 79, 065004 (2009). [arXiv:0808.1931 [gr-qc]]. 


\section{A.2 Kodama time: Geometrically preferred foliations of spherically symmetric space- times}

Tn a general time-dependent (3+1)-dimensional spherically symmetric spacetime, the so-called Kodama vector is a naturally defined geometric quantity that is timelike outside the evolving horizon and so defines a preferred class of fiducial observers. However the Kodama vector does not by itself define any preferred notion of time. We first extract as much information as possible by invoking the "warped product" structure of spherically symmetric spacetime to study the Kodama vector, and the associated Kodama energy flux, in a coordinate independent manner. Using this formalism we construct a general class of conservation laws, generalizing Kodama's energy flux.

We then demonstrate that a preferred time coordinate - which we shall call Kodama time - can be introduced by taking the additional step of applying the Clebsch decomposition theorem to the Kodama vector. We thus construct a geometrically preferred coordinate system for any timedependent spherically symmetric spacetime, and explore its properties. We study the geometrically preferred fiducial observers, and demonstrate that it is possible to define and calculate a generalized notion of surface gravity that is valid throughout the entire evolving spacetime. Furthermore, by building and suitably normalizing set of radial null geodesics, we can show that this generalized surface gravity passes several consistency tests and has a physically appropriate static limit.

Phys. Rev. D 82, 044027 (2010). [arXiv:1004.1456 [gr-qc]]. 


\section{A.3 Tolman mass, generalized surface gravity, and entropy bounds}

Tn any static spacetime the quasi-local Tolman mass contained within a volume can be reduced to a Gauss-like surface integral involving the flux of a suitably defined generalized surface gravity. By introducing some basic thermodynamics and invoking the Unruh effect one can then develop elementary bounds on the quasi-local entropy that are very similar in spirit to the holographic bound, and closely related to entanglement entropy.

Phys. Rev. Lett. 105, 041302 (2010). [arXiv:1005.1132 [gr-qc]].

\section{A.4 Entropy bounds for uncollapsed rotating bodies}

Zntropy bounds in black hole physics, based on a wide variety of differ-1 ent approaches, have had a long and distinguished history. Recently the current authors have turned attention to uncollapsed systems and obtained a robust entropy bound for uncollapsed static spherically symmetric configurations. In the current article we extend this bound to rotating systems. This extension is less simple than one might at first suppose.

Purely classically, (using only classical general relativity and basic thermodynamics), it is possible to show that the entropy of uncollapsed matter inside a region enclosed by a surface of area $\mathscr{A}$ is bounded from above by

$$
S \leq \frac{\|\vec{\kappa}\|_{\max (\text { surface })} \mathscr{A}}{4 \pi T_{\infty}}
$$

Here $\vec{\kappa}$ is a suitably defined surface gravity. By appealing to the Unruh effect, which is our only invocation of quantum physics, we argue that for a suitable class of fiducial observers there is a lower bound on the temper- 
ature (as measured at spatial infinity)

$$
T_{\infty} \geq \max _{\text {FIDOs }}\left\{\frac{\|\vec{\kappa}\|}{2 \pi}\right\} .
$$

Thus, using only classical general relativity, basic thermodynamics, and the Unruh effect, we are able to argue that for uncollapsed matter

$$
S \leq \frac{1}{2} \mathscr{A}
$$

JHEP 1103, 056 (2011). [arXiv:1011.4538 [gr-qc]].

\section{A.5 Some generalizations of the Raychaudhuri equation}

$\mathrm{T}$

The Raychaudhuri equation has seen extensive use in general relativ-

ity, most notably in the development of various singularity theorems. In this rather technical article we shall generalize the Raychaudhuri equation in several ways. First an improved version of the standard timelike Raychaudhuri equation is developed, where several key terms are lumped together as a divergence. This already has a number of interesting applications, both within the ADM formalism and elsewhere. Second, a spacelike version of the Raychaudhuri equation is briefly discussed. Third, a version of the Raychaudhuri equation is developed that does not depend on the use of normalized congruences. This leads to useful formulae for the "diagonal" part of the Ricci tensor. Fourth, a "two vector" version of the Raychaudhuri equation is developed that uses two congruences to effectively extract "off diagonal" information concerning the Ricci tensor.

Phys. Rev. D 83, 104016 (2011). arXiv:1012.4806 [gr-qc]. 


\section{Bibliography}

[1] H. Kodama, "Conserved energy flux for the spherically symmetric system and the back reaction problem in the black hole evaporation," Prog. Theor. Phys., vol. 63, p. 1217, 1980.

[2] S. H. Lamb, Hydrodynamics. Dover, 1945.

[3] S. Deser, R. Jackiw, and A. P. Polychronakos, "Clebsch (string) parameterization of 3-vectors and their actions," Phys. Lett., vol. A279, pp. 151-153, 2001.

[4] S. E. Perez-Bergliaffa, K. Hibberd, M. Stone, and M. Visser, "Wave equation for sound in fluids with vorticity," Physica D: Nonlinear Phenomena, vol. 191, no. 1-2, pp. 121-136, 2004.

[5] S. A. Hayward, "General laws of black hole dynamics," Phys. Rev., vol. D49, pp. 6467-6474, 1994.

[6] S. A. Hayward, "Gravitational energy in spherical symmetry," Phys. Rev., vol. D53, pp. 1938-1949, 1996.

[7] S. A. Hayward, "Unified first law of black-hole dynamics and relativistic thermodynamics," Class. Quant. Grav., vol. 15, pp. 3147-3162, 1998.

[8] S. A. Hayward, "Quasilocal gravitational energy," Phys. Rev., vol. D49, pp. 831-839, 1994.

[9] A. B. Nielsen and J. H. Yoon, "Dynamical surface gravity," Class. Quant. Grav., vol. 25, p. 085010, 2008.

[10] A. B. Nielsen and D. H. Yeom, "Spherically symmetric trapping horizons, the Misner-Sharp mass and black hole evaporation," Int. J. Mod. Phys., vol. A24, pp. 5261-5285, 2009. 
[11] A. B. Nielsen, "Black Holes without Event Horizons," J. Korean Phys. Soc., vol. 54, pp. 2576-2582, 2009.

[12] A. B. Nielsen, "Black holes and black hole thermodynamics without event horizons," Gen. Rel. Grav., vol. 41, pp. 1539-1584, 2009.

[13] A. B. Nielsen, "Black holes as local horizons," e-Print: arXiv: 0711.0313 [gr-qc], 2007.

[14] A. B. Nielsen, "Black holes without boundaries," Int. J. Mod. Phys., vol. D17, pp. 2359-2366, 2009.

[15] A. B. Nielsen and M. Visser, "Production and decay of evolving horizons," Class. Quant. Grav., vol. 23, pp. 4637-4658, 2006.

[16] M. Visser, "Dirty black holes: Thermodynamics and horizon structure," Phys. Rev., vol. D46, pp. 2445-2451, 1992.

[17] H. Maeda and M. Nozawa, "Generalized Misner-Sharp quasilocal mass in Einstein-Gauss-Bonnet gravity," Phys. Rev., vol. D77, p. 064031, 2008.

[18] H. Maeda, "Gauss-Bonnet black holes with non-constant curvature horizons," Phys. Rev., vol. D81, p. 124007, 2010.

[19] B. O'Neill, Semi-Riemannian geometry (with applications to relativity). Academic Press, 1983.

[20] J. Carot and J. da Costa, "On the geometry of warped spacetimes," Class. Quant. Grav., no. 10, pp. 461-482, 1993.

[21] Y. Agaoka, I. B. Kim, B. H. Kim, and D. J. Yeom, “On doubly warped product manifolds," Mem. Fac. Integrated Arts and Sci., Hiroshima Univ., Ser. IV, 24 (Dec 1998) 1-10., 1998.

[22] Z. Sentürk, "Differential geometry and its applications," Proc. Conf. Prauge, 2004. 
[23] W. C. Hernandez and C. W. Misner, "Observer time as a coordinate in relativistic spherical hydrodynamics," Astrophys. J., vol. 143, no. 152, 1966.

[24] C. W. Misner and D. H. Sharp, "Relativistic equations for adiabatic, spherically symmetric gravitational collapse," Phys. Rev., vol. 136, pp. B571-B576, 1964.

[25] K. S. Thorne, R. H. Price, and D. A. Macdonald, Black Holes: The membrane paradigm. Yale University Press, 1986.

[26] I. Racz, "On the use of the Kodama vector field in spherically symmetric dynamical problems," Class. Quant. Grav., vol. 23, pp. 115$124,2006$.

[27] P. Csizmadia and I. Racz, "Gravitational collapse and topology change in spherically symmetric dynamical systems," Class. Quant. Grav., vol. 27, p. 015001, 2010.

[28] S. M. Carroll, An Introduction to General Relativity Spacetime and Geometry. Addison-Wesley, San Francisco, 2004.

[29] R. M. Wald, General Relativity. University of Chicago Press, 1984.

[30] J. D. Brown and J. W. York, Jr., "Quasilocal energy and conserved charges derived from the gravitational action," Phys. Rev., vol. D47, pp. 1407-1419, 1993.

[31] M. Visser, Traversable wormholes: From Einstein to Hawking. AIP Press/Springer Verlag, New York, 1995.

[32] A. Ashtekar and B. Krishnan, "Isolated and dynamical horizons and their applications," Living Rev. Rel., vol. 7, p. 10, 2004.

[33] A. Ashtekar, J. Engle, T. Pawlowski, and C. Van Den Broeck, "Multipole moments of isolated horizons," Class. Quant. Grav., vol. 21, pp. 2549-2570, 2004. 
[34] A. Ashtekar and G. J. Galloway, "Some uniqueness results for dynamical horizons," Adv. Theor. Math. Phys., vol. 9, pp. 1-30, 2005.

[35] M. Visser, "Essential and inessential features of Hawking radiation," Int. J. Mod. Phys., vol. D12, pp. 649-661, 2003.

[36] C. Barceló, S. Liberati, S. Sonego, and M. Visser, "Hawking-like radiation does not require a trapped region," Phys. Rev. Lett., vol. 97, p. 171301, 2006.

[37] C. Barceló, S. Liberati, S. Sonego, and M. Visser, “Quasi-particle creation by analogue black holes," Class. Quant. Grav., vol. 23, pp. 5341$5366,2006$.

[38] S. C. Anco, "Mean curvature flow and quasilocal mass for twosurfaces in hamiltonian general relativity," J.Math.Phys., vol. 48, p. 052502, 2007.

[39] J. M. Senovilla, "Novel results on trapped surfaces," 2nd Conference On Mathematics Of Gravitation. e-print: arXiv: 0311005 [gr-qc], 2003.

[40] J. M. Senovilla, "Trapped submanifolds in Lorentzian geometry," 13th Fall Workshop On Geometry And Physics. e-print: arXiv: 0412256 [math-dg], 2004.

[41] G. J. Galloway and J. M. Senovilla, "Singularity theorems based on trapped submanifolds of arbitrary co-dimension," Class.Quant.Grav., vol. 27, p. 152002, 2010.

[42] R. C. Tolman, Relativity, thermodynamics, and cosmology. Dover, 1987.

[43] J. M. Bardeen, B. Carter, and S. W. Hawking, "The Four laws of black hole mechanics," Commun. Math. Phys., vol. 31, pp. 161-170, 1973.

[44] W. G. Unruh, "Notes on black hole evaporation," Phys. Rev., vol. D14, p. 870, 1976. 
[45] R. Bousso, “The holographic principle," Rev. Mod. Phys., vol. 74, pp. 825-874, 2002.

[46] J. D. Bekenstein, "Generalized second law of thermodynamics in black hole physics," Phys. Rev., vol. D9, pp. 3292-3300, 1974.

[47] J. D. Bekenstein, "A Universal Upper Bound on the Entropy to Energy Ratio for Bounded Systems," Phys. Rev., vol. D23, p. 287, 1981.

[48] T. Padmanabhan, "Gravitational entropy of static spacetimes and microscopic density of states," Class. Quant. Grav., vol. 21, pp. 44854494, 2004.

[49] T. Padmanabhan, "Surface Density of Spacetime Degrees of Freedom from Equipartition Law in theories of Gravity," Phys. Rev., vol. D81, p. 124040, 2010.

[50] S. Kolekar and T. Padmanabhan, "Holography in Action," Phys. Rev., vol. D82, p. 024036, 2010.

[51] M. Srednicki, "Entropy and area," Phys. Rev. Lett., vol. 71, pp. 666669, 1993.

[52] A. Komar, "Covariant conservation laws in general relativity," Phys. Rev., vol. 113, pp. 934-936, 1959.

[53] L. D. Landau and E. M. Lifschitz., The Classical Theory of Fields. Pergamon Press, Oxford, 4th revised english ed., 2000.

[54] R. D. Sorkin, R. M. Wald, and Z. J. Zhang, "Entropy of selfgravitating radiation," Gen. Rel. Grav., vol. 13, pp. 1127-1146, 1981.

[55] S. D. H. Hsu and D. Reeb, "Black hole entropy, curved space and monsters," Phys. Lett., vol. B658, pp. 244-248, 2008. 
[56] S. D. H. Hsu and D. Reeb, "Monsters, black holes and the statistical mechanics of gravity," Mod. Phys. Lett., vol. A24, pp. 1875-1887, 2009.

[57] P. O. Mazur and E. Mottola, "Gravitational condensate stars," ePrint: arXiv: 0109035 [gr-qc], 2001.

[58] P. O. Mazur and E. Mottola, "Gravitational vacuum condensate stars," Proc. Nat. Acad. Sci., vol. 101, pp. 9545-9550, 2004.

[59] M. Visser and D. L. Wiltshire, "Stable gravastars - an alternative to black holes?," Class. Quant. Grav., vol. 21, pp. 1135-1152, 2004.

[60] C. Cattoën, T. Faber, and M. Visser, "Gravastars must have anisotropic pressures," Class. Quant. Grav., vol. 22, pp. 4189-4202, 2005.

[61] C. Barceló, S. Liberati, S. Sonego, and M. Visser, "Fate of gravitational collapse in semiclassical gravity," Phys. Rev., vol. D77, p. $044032,2008$.

[62] C. Barceló, S. Liberati, S. Sonego, and M. Visser, "Revisiting the semiclassical gravity scenario for gravitational collapse," AIP Conf. Proc., vol. 1122, pp. 99-106, 2009.

[63] C. Barceló, S. Liberati, S. Sonego, and M. Visser, "Black stars, not holes," Sci. Am., Oct. 2009.

[64] M. Visser, C. Barceló, S. Liberati, and S. Sonego, "Small, dark, and heavy: But is it a black hole?," PoS, vol. BHSGRANDSTRINGS2008, p. 010, 2008.

[65] J. P. S. Lemos and O. B. Zaslavskii, "Entropy of quasiblack holes," Phys. Rev., vol. D81, p. 064012, 2010. 
[66] J. P. S. Lemos and O. B. Zaslavskii, "Black hole mimickers: regular versus singular behavior," Phys. Rev., vol. D78, p. 024040, 2008.

[67] R. C. Tolman and P. Ehrenfest, "Temperature Equilibrium in a Static Gravitational Field," Phys. Rev., vol. 36, pp. 1791-1798, 1930.

[68] R. C. Tolman, "Thermal Equilibrium in a General Gravitational Field," Proc. Nat. Acad. Sci., vol. 21, pp. 321-326, Jun 1935.

[69] N. L. Balazs, “On relativistic thermodynamics," Ap. J., vol. 128, pp. 398-405, 1958.

[70] G. Abreu and M. Visser, "Kodama time: Geometrically preferred foliations of spherically symmetric spacetimes," Phys. Rev., vol. D82, p. $044027,2010$.

[71] G. Abreu and M. Visser, "Tolman mass, generalized surface gravity, and entropy bounds," Phys. Rev. Lett., vol. 105, p. 041302, 2010.

[72] G. Abreu and M. Visser, "Entropy bounds for uncollapsed matter," ERE2010. e-Print: arXiv: 1011.4538 [gr-qc], 2010.

[73] G. Abreu and M. Visser, "Entropy bounds for uncollapsed rotating bodies," JHEP, vol. 2011, no. 3, p. 56, 2011.

[74] J. B. Hartle, "Slowly rotating relativistic stars. IV. Rotational energy and moment of inertial for stars in differential rotation," Astrophys. J., no. 161, pp. 111-118, 1970.

[75] W. Israel, "Nonstationary irreversible thermodynamics: A causal relativistic theory," Ann. Phys., vol. 100, pp.310-331, 1976.

[76] W. M. Suen and K. Young, "Thermodynamic equilibrium in relativistic rotating systems," Classical and Quantum Gravity, no. 5, pp. 1447-1457, 1998. 
[77] N. Andersson, K. D. Kokkotas, and N. Stergioulas, "On the relevance of the r-mode instability for accreting neutron stars and white dwarfs," Astrophys. J., vol. 516, p. 307, 1999.

[78] N. Andersson and K. D. Kokkotas, "The r-mode instability in rotating neutron stars," Int. J. Mod. Phys., vol. D10, pp. 381-442, 2001.

[79] N. Andersson, D. I. Jones, K. D. Kokkotas, and N. Stergioulas, "Rmode runaway and rapidly rotating neutron stars," Astrophys. J., vol. 534, p. L75, 2000.

[80] O. Klein, “Thermal equilibrium in a general gravitational field," Rev. Mod. Phys., no. 21, pp. 531-533, 1949.

[81] R. C. Tolman, "On the use of the energy-momentum principle in general relativity," Phys. Rev., no. 35, p. 875, 1930.

[82] C. Barceló, S. Liberati, S. Sonego, and M. Visser, "Minimal conditions for the existence of a Hawking-like flux," Phys. Rev., vol. D83, no. 041501(R), 2011.

[83] C. Barceló, S. Liberati, S. Sonego, and M. Visser, "Hawking-like radiation from evolving black holes and compact horizonless objects," JHEP, vol. 1102, no. 2, pp. 1-30, 2011.

[84] S. W. Hawking, “Black hole explosions?," Nature, vol. 248, pp. 30-31, 1974.

[85] S. W. Hawking, "Particle creation by black holes," Commun. Math. Phys., vol. 43, no. 199, 1975.

[86] S. Weinfurtner, E. W. Tedford, M. C. J. Penrice, W. G. Unruh, and G. A. Lawrence, "Measurement of stimulated Hawking emission in an analogue system," Phys. Rev. Lett, vol. 106, no. 021302, 2011. 
[87] F. Belgiorno, S. L. Cacciatori, M. Clerici, V. Gorini, G. Ortenzi, L. Rizzi, E. Rubino, V. G. Sala, and D. Faccio, "Hawking radiation from ultrashort laser pulse filaments," Phys. Rev. Lett., vol. 105, no. 203901, 2010.

[88] F. Belgiorno, S. L. Cacciatori, M. Clerici, V. Gorini, G. Ortenzi, L. Rizzi, E. Rubino, V. G. Sala, and D. Faccio, "Reply to Comment on: Hawking radiation from ultrashort laser pulse filaments," e-Print: arXiv: 1012.5062 [quant-ph], 2010.

[89] R. Schützhold and W. G. Unruh, "Comment on: Hawking radiation from ultrashort laser pulse filaments," e-Print: arXiv:1012.2686 [quant-ph], 2010.

[90] L. Smarr, "Mass formula for Kerr black holes," Phys. Rev. Lett., vol. 30, pp. 71-73, 1973.

[91] C. Barceló, Private communication.

[92] S. W. Hawking and G. F. R. Ellis, The Large scale structure of space-time. Cambridge University Press, 1973.

[93] E. Poisson, A relativist's toolkit: The mathematics of black hole mechanics. Cambridge University Press, 2004.

[94] T. Padmanabhan and A. Patel, "Semiclassical quantization of gravity. 1. Entropy of horizons and the area spectrum," e-Print: arXiv: 0305165 [hep-th], 2003.

[95] T. Padmanabhan and A. Patel, "Role of horizons in semiclassical gravity: Entropy and the area spectrum," e-Print: arXiv: 0309053 $[g r-q c], 2003$.

[96] C. W. Misner, K. S. Thorne, and J. A. Wheeler, Gravitation. Freeman, San Francisco, 1973. 
[97] A. D. Rendall, Partial differential equations in general relativity. Oxford University Press, 2008.

[98] R. P. Kerr, "Gravitational field of a spinning mass as an example of algebraically special metrics," Phys. Rev. Lett., no. 11, p. 237, 1963.

[99] R. P. Kerr, "Gravitational collapse and rotation," First Texas Symposium on Relativistic Astrophysics, December 1963.

[100] D. L. Wiltshire, M. Visser, and S. M. Scott, The Kerr spacetime: Rotating black holes in general relativity. Cambridge University Press, 2009.

[101] M. Visser, “The Kerr spacetime: A brief introduction," e-Print: arXiv: $0706.0622[g r-q c], 2007$.

[102] H. Epstein, V. Glaser, and A. Jaffe, "Nonpositivity of the energy density in quantized field theories," Nuovo Cimento 36, no. 36, p. 1016, 1965.

[103] T. A. Roman, "Quantum stress energy tensors and the weak energy condition," Phys. Rev., vol. D33, pp. 3526-3533, 1986.

[104] C. Barceló and M. Visser, "Twilight for the energy conditions?," Int. J. Mod. Phys., vol. D11, pp. 1553-1560, 2002.

[105] M. Alcubierre, "The warp drive: hyper-fast travel within general relativity," Class. Quant. Grav., vol. 11, pp. L73-L77, 1994.

[106] M. J. Pfénning and L. H. Ford, "The unphysical nature of 'warp drive'," Class. Quant. Grav., vol. 14, pp. 1743-1751, 1997.

[107] A. E. Everett and T. A. Roman, "A superluminal subway: The Krasnikov tube," Phys. Rev., vol. D56, pp. 2100-2108, 1997.

[108] F. S. N. Lobo and M. Visser, "Linearized warp drive and the energy conditions," e-Print: arXiv: 0412065 [gr-qc], 2004. 
[109] F. S. N. Lobo and M. Visser, "Fundamental limitations on 'warp drive' spacetimes," Class. Quant. Grav., vol. 21, pp. 5871-5892, 2004.

[110] M. S. Morris and K. S. Thorne, "Wormholes in space-time and their use for interstellar travel: A tool for teaching general relativity," Am. J. Phys., vol. 56, pp. 395-412, 1988.

[111] M. S. Morris, K. S. Thorne, and U. Yurtsever, “Wormholes, Time Machines, and the Weak Energy Condition," Phys. Rev. Lett., vol. 61, pp. 1446-1449, 1988.

[112] M. Visser, "Traversable wormholes from surgically modified Schwarzschild space-times," Nucl. Phys., vol. B328, pp. 203-212, 1989.

[113] M. Visser, "Traversable wormholes: Some simple examples," Phys. Rev., vol. D39, pp. 3182-3184, 1989.

[114] M. Visser, S. Kar, and N. Dadhich, "Traversable wormholes with arbitrarily small energy condition violations," Phys. Rev. Lett., vol. 90, p. 201102, 2003.

[115] S. Kar, N. Dadhich, and M. Visser, "Quantifying energy condition violations in traversable wormholes," Pramana, vol. 63, pp. 859-864, 2004.

[116] C. J. Fewster and T. A. Roman, "On wormholes with arbitrarily small quantities of exotic matter," Phys. Rev., vol. D72, p. 044023, 2005.

[117] C. J. Fewster and T. A. Roman, "Problems with wormholes which involve arbitrarily small amounts of exotic matter," e-Print: arXiv: 0510079 [gr-qc], 2005.

[118] T. A. Roman, "Inflating Lorentzian wormholes," Phys. Rev., vol. D47, pp. 1370-1379, 1993. 
[119] T. A. Roman and P. G. Bergmann, "Stellar collapse without singularities?," Phys. Rev., vol. D28, pp. 1265-1277, 1983.

[120] J. G. Cramer, R. L. Forward, M. S. Morris, M. Visser, G. Benford, and G. A. Landis, "Natural wormholes as gravitational lenses," Phys. Rev., vol. D51, pp. 3117-3120, 1995.

[121] L. H. Ford and T. A. Roman, "'Cosmic flashing' in four-dimensions," Phys. Rev., vol. D46, pp. 1328-1339, 1992.

[122] L. H. Ford and T. A. Roman, "Moving mirrors, black holes and cosmic censorship," Phys. Rev., vol. D41, p. 3662, 1990.

[123] J. Friedman et al., "Cauchy problem in space-times with closed timelike curves," Phys. Rev., vol. D42, pp. 1915-1930, 1990.

[124] S. W. Hawking, "The chronology protection conjecture," Phys. Rev., vol. D46, pp. 603-611, 1992.

[125] M. Visser, "The quantum physics of chronology protection," e-Print: arXiv: 0204022 [gr-qc], 2002.

[126] M. Visser, "The reliability horizon for semi-classical quantum gravity: Metric fluctuations are often more important than backreaction," Phys. Lett., vol. B415, pp. 8-14, 1997.

[127] M. Visser, "From wormhole to time machine: Comments on Hawking's chronology protection conjecture," Phys. Rev., vol. D47, pp. 554-565, 1993.

[128] M. Visser, "Hawking's chronology protection conjecture: Singularity structure of the quantum stress energy tensor," Nucl. Phys., vol. B416, pp. 895-906, 1994.

[129] T. A. Roman, "Some thoughts on energy conditions and wormholes," e-Print: arXiv: 0409090 [gr-qc], 2004. 
[130] E. E. Flanagan and R. M. Wald, "Does backreaction enforce the averaged null energy condition in semiclassical gravity?," Phys. Rev., vol. D54, pp. 6233-6283, 1996.

[131] C. J. Fewster and T. A. Roman, "Null energy conditions in quantum field theory," Erratum-ibid., vol. D80, p. 069903, 2009.

[132] C. J. Fewster, K. D. Olum, and M. J. Pfenning, "Averaged null energy condition in spacetimes with boundaries," Phys. Rev., vol. D75, p. 025007, 2007.

[133] L. H. Ford, A. D. Helfer, and T. A. Roman, "Spatially averaged quantum inequalities do not exist in four-dimensional spacetime," Phys. Rev., vol. D66, p. 124012, 2002.

[134] M. Visser, "Scale anomalies imply violation of the averaged null energy condition," Phys. Lett., vol. B349, pp. 443-447, 1995.

[135] L. H. Ford, "Constraints on negative energy fluxes," Phys. Rev., vol. D43, pp. 3972-3978, 1991.

[136] L. H. Ford and T. A. Roman, "Averaged energy conditions and quantum inequalities," Phys. Rev., vol. D51, pp. 4277-4286, 1995.

[137] L. H. Ford and T. A. Roman, "Quantum Field Theory Constrains Traversable Wormhole Geometries," Phys. Rev., vol. D53, pp. 54965507, 1996.

[138] L. H. Ford and T. A. Roman, "Motion of inertial observers through negative energy," Phys. Rev., vol. D48, pp. 776-782, 1993.

[139] L. H. Ford and T. A. Roman, "Restrictions on negative energy density in flat spacetime," Phys. Rev., vol. D55, pp. 2082-2089, 1997.

[140] L. H. Ford, M. J. Pfenning, and T. A. Roman, "Quantum inequalities and singular negative energy densities," Phys. Rev., vol. D57, pp. 4839-4846, 1998. 
[141] A. Borde, L. H. Ford, and T. A. Roman, "Constraints on spatial distributions of negative energy," Phys. Rev., vol. D65, p. 084002, 2002.

[142] C. J. Fewster and L. W. Osterbrink, "Quantum Energy Inequalities for the Non-Minimally Coupled Scalar Field," J. Phys., vol. A41, p. 025402, 2008.

[143] C. J. Fewster, "Quantum energy inequalities in two dimensions," Phys. Rev., vol. D70, p. 127501, 2004.

[144] E. E. Flanagan, "Quantum inequalities in two dimensional curved spacetimes," Phys. Rev., vol. D66, p. 104007, 2002.

[145] E. E. Flanagan, "Quantum inequalities in two dimensional Minkowski spacetime," Phys. Rev., vol. D56, pp. 4922-4926, 1997.

[146] C. J. Fewster and S. P. Eveson, "Bounds on negative energy densities in flat spacetime," Phys. Rev., vol. D58, p. 084010, 1998.

[147] L. H. Ford and T. A. Roman, "The quantum interest conjecture," Phys. Rev., vol. D60, p. 104018, 1999.

[148] C. J. Fewster and E. Teo, "Quantum inequalities and 'quantum interest' as eigenvalue problems," Phys. Rev., vol. D61, p. 084012, 2000.

[149] E. Teo and K. F. Wong, "Quantum interest in two dimensions," Phys. Rev., vol. D66, p. 064007, 2002.

[150] B. Simon, "The Bound State of Weakly Coupled Schrodinger Operators in One and Two-Dimensions," Annals Phys., vol. 97, pp. 279288, 1976.

[151] F. Pretorius, "Quantum interest for scalar fields in Minkowski spacetime," Phys. Rev., vol. D61, p. 064005, 2000. 